\title{
Representations of Finite Groups
}

\author{
Jeremy Sylvestre \\ University of Alberta, Augustana Campus \\ jeremy.sylvestre@ualberta.ca
}

October 27, 2022

\begin{abstract}
We provide a formal framework for the theory of representations of finite groups, as modules over the group ring. Along the way, we develop the general theory of groups (relying on the group_add class for the basics), modules, and vector spaces, to the extent required for theory of group representations. We then provide formal proofs of several important introductory theorems in the subject, including Maschke's theorem, Schur's lemma, and Frobenius reciprocity. We also prove that every irreducible representation is isomorphic to a submodule of the group ring, leading to the fact that for a finite group there are only finitely many isomorphism classes of irreducible representations. In all of this, no restriction is made on the characteristic of the ring or field of scalars until the definition of a group representation, and then the only restriction made is that the characteristic must not divide the order of the group.
\end{abstract}

\section{Contents}

1 Preliminaries 5

1.1 Logic . . . . . . . . . . . . . . . . . . . . 5

1.2 Sets .......................... 5

1.3 Lists . . . . . . . . . . . . . . . . . 5

1.3.1 zip ..................... 5

1.3 .2 concat.................. 6

1.3.3 strip-while................ 6

1.3.4 sum-list ................. 6

1.3 .5 listset ................. 7

1.4 Functions ..................... 8

1.4.1 Miscellaneous facts . . . . . . . . . . . 8

1.4.2 Support of a function ............. 8

1.4.3 Convolution ................. . . . 9

1.5 Almost-everywhere-zero functions . . . . . . . . . . . 10 
1.5.1 Definition and basic properties . . . . . . . . 10

1.5.2 Delta (impulse) functions . . . . . . . . . . . 10

1.5.3 Convolution of almost-everywhere-zero functions . . . 11

1.5.4 Type definition, instantiations, and instances . . . . 12

1.5.5 Transfer facts . . . . . . . . . . . . 15

1.5.6 Almost-everywhere-zero functions with constrained sup-



1.6 Polynomials . . . . . . . . . . . . . . . . . . . . . . . . 18

1.7 Algebra of sets . . . . . . . . . . . . . . . . 19

1.7.1 General facts . . . . . . . . . . . . . . . . . 19

1.7.2 Additive independence of sets . . . . . . . . . . . 19

1.7.3 Inner direct sums . . . . . . . . . . . . . . . 20

2 Groups 21

2.1 Locales and basic facts . . . . . . . . . . . . . 21

2.1.1 Locale Group and finite variant FinGroup . . . . . . . 21

2.1.2 Abelian variant locale AbGroup . . . . . . . . . . 23

2.2 Right cosets . . . . . . . . . . . . . . . . . 23

2.3 Group homomorphisms . . . . . . . . . . . . 25

2.3 .1 Preliminaries . . . . . . . . . . . . 25

2.3 .2 Locales . . . . . . . . . . . . . . . . 25

2.3 .3 Basic facts . . . . . . . . . . . 26

2.3.4 Basic facts about endomorphisms . . . . . . . . 27

2.3.5 Basic facts about isomorphisms . . . . . . . . . 28

2.3 .6 Hom-sets . . . . . . . . . . . . . . . 28

2.4 Facts about collections of groups . . . . . . . . . . . 29

2.5 Inner direct sums of Abelian groups . . . . . . . . . . 30

2.5.1 General facts . . . . . . . . . . . . 30

2.5.2 Element decomposition and projection . . . . . . . 30

2.6 Rings . . . . . . . . . . . . . . . . . . . 32

2.6.1 Preliminaries . . . . . . . . . . . . . . 32

2.6.2 Locale and basic facts . . . . . . . . . . . . 32

2.7 The group ring . . . . . . . . . . . . . . . . 33

2.7.1 Definition and basic facts . . . . . . . . . 33

2.7.2 Projecting almost-everywhere-zero functions onto a group ring ...................... 34

3 Modules 35

3.1 Locales and basic facts . . . . . . . . . . . . . 35

3.1 .1 Locales . . . . . . . . . . . . . . . . . 35

3.1 .2 Basic facts . . . . . . . . . . . 36

3.1.3 Module and submodule instances . . . . . . . . . 38

3.2 Linear algebra in modules . . . . . . . . . . . . . . . 39

3.2.1 Linear combinations: lincomb . . . . . . . . . . . 39 
3.2.2 Spanning: RSpan and Span . . . . . . . . . . . . 41

3.2 .3 Finitely generated modules . . . . . . . . . . . . 44

3.2.4 R-linear independence . . . . . . . . . . . . 45

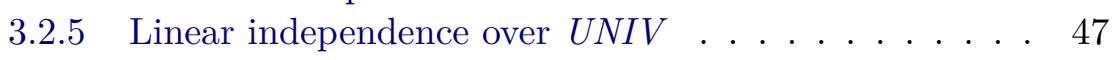

3.2.6 Rank . . . . . . . . . . . . . . . . . 48

3.3 Module homomorphisms . . . . . . . . . . . . . . . 49

3.3.1 Locales . . . . . . . . . . . . . . . . . . . . . 49

3.3 .2 Basic facts . . . . . . . . . . . . 50

3.3.3 Basic facts about endomorphisms . . . . . . . . 51

3.3.4 Basic facts about isomorphisms . . . . . . . . . 52

3.4 Inner direct sums of RModules . . . . . . . . . . . . 52

4 Vector Spaces $\quad \mathbf{5 2}$

4.1 Locales and basic facts . . . . . . . . . . . . . 52

4.2 Linear algebra in vector spaces . . . . . . . . . . . 54

4.2.1 Linear independence and spanning . . . . . . . . . 54

4.2.2 Basis for a vector space: basis-for . . . . . . . . 55

4.3 Finite dimensional spaces . . . . . . . . . . . . 56

4.4 Vector space homomorphisms . . . . . . . . . . 57

4.4 Locales . . . . . . . . . . . . . . . . 57

4.4 .2 Basic facts . . . . . . . . . . . . 58

4.4 .3 Hom-sets . . . . . . . . . . . . . . . . 59

4.4.4 Basic facts about endomorphisms . . . . . . . . . 60

4.4.5 Polynomials of endomorphisms . . . . . . . . . 63

4.4.6 Existence of eigenvectors of endomorphisms of finitedimensional vector spaces . . . . . . . . . . . 64

5 Modules Over a Group Ring $\quad 64$

5.1 Almost-everywhere-zero functions as scalars . . . . . . . . . 64

5.2 Locale and basic facts . . . . . . . . . . . . . 65

5.3 Modules over a group ring as a vector spaces . . . . . . . 68

5.4 Homomorphisms of modules over a group ring . . . . . . . . 69

5.4 .1 Locales . . . . . . . . . . . . . . . . . 69

5.4 .2 Basic facts . . . . . . . . . . . . . . 69

5.4.3 Basic facts about endomorphisms . . . . . . . . . 72

5.4.4 Basic facts about isomorphisms . . . . . . . . . 72

5.4 .5 Hom-sets . . . . . . . . . . . . . . . . 74

5.5 Induced modules . . . . . . . . . . . . . . 75

5.5.1 Additive function spaces . . . . . . . . . . 75

5.5.2 Spaces of functions which transform under scalar multiplication by almost-everywhere-zero functions . . . . 76

5.5.3 General induced spaces of functions on a group ring . 76

5.5 .4 The right regular action . . . . . . . . . . 78

5.5.5 Locale and basic facts . . . . . . . . . . . . 78 
6 Representations of Finite Groups $\quad 81$

6.1 Locale and basic facts . . . . . . . . . . . . . . 81

6.2 Irreducible representations . . . . . . . . . . . . . . 82

6.3 Maschke's theorem . . . . . . . . . . . . . . . 83

6.3.1 Averaged projection onto a G-subspace . . . . . . . . 83

6.3 .2 The theorem ................ . . 84

6.3.3 Consequence: complete reducibility . . . . . . . . . 84

6.3.4 Consequence: decomposition relative to a homomomorphism . . . . . . . . . . . . 85

6.4 Schur's lemma . . . . . . . . . . . . . . 85

6.5 The group ring as a representation space . . . . . . . 85

6.5.1 The group ring is a representation space . . . . . . 85

6.5.2 Irreducible representations are constituents of the group ring . . . . . . . . . . . . 86

6.6 Isomorphism classes of irreducible representations . . . . . . 86

6.7 Induced representations . . . . . . . . . . . . . 88

6.7.1 Locale and basic facts . . . . . . . . . . . . 88

6.7.2 A basis for the induced space . . . . . . . . . 89

6.7.3 The induced space is a representation space . . . . . . 90

6.8 Frobenius reciprocity . . . . . . . . . . . . . . . . 91

6.8.1 Locale and basic facts . . . . . . . . . . . . . 91

6.8.2 The required isomorphism of Hom-sets . . . . . . . . . 92

6.8.3 The inverse map of Hom-sets . . . . . . . . . . . . . 93

6.8.4 Demonstration of bijectivity . . . . . . . . . . 94

6.8.5 The theorem . . . . . . . . . . . . . . 94

$\begin{array}{lll}7 & \text { Bibliography } & 95\end{array}$

Note: A number of the proofs in this theory were modelled on or inspired by proofs in the books listed in the bibliography.

\author{
theory Rep-Fin-Groups \\ imports \\ HOL-Library.Function-Algebras \\ HOL-Library.Set-Algebras \\ HOL-Computational-Algebra.Polynomial \\ begin
}




\section{Preliminaries}

In this section, we establish some basic facts about logic, sets, and functions that are not available in the HOL library. As well, we develop some theory for almost-everywhere-zero functions in preparation of the definition of the group ring.

\subsection{Logic}

lemma conjcases [case-names BothTrue OneTrue OtherTrue BothFalse] : assumes BothTrue: $P \wedge Q \Longrightarrow R$

and OneTrue: $P \wedge \neg Q \Longrightarrow R$

and OtherTrue: $\neg P \wedge Q \Longrightarrow R$

and BothFalse: $\neg P \wedge \neg Q \Longrightarrow R$

shows $R$

$\langle$ proof $\rangle$

\subsection{Sets}

lemma empty-set-diff-single : $A-\{x\}=\{\} \Longrightarrow A=\{\} \vee A=\{x\}$

$\langle$ proof $\rangle$

lemma seteqI $:(\bigwedge a . a \in A \Longrightarrow a \in B) \Longrightarrow(\bigwedge b . b \in B \Longrightarrow b \in A) \Longrightarrow A=B$ $\langle$ proof $\rangle$

lemma prod-ballI : $(\bigwedge a b .(a, b) \in A x B \Longrightarrow P a b) \Longrightarrow \forall(a, b) \in A x B . P a b$ $\langle$ proof $\rangle$

lemma good-card-imp-finite : of-nat (card $A) \neq\left(0::^{\prime} a::\right.$ semiring- 1$) \Longrightarrow$ finite $A$ $\langle$ proof $\rangle$

\section{$1.3 \quad$ Lists}

1.3.1 zip

lemma zip-truncate-left : zip xs ys $=$ zip (take (length ys) xs) ys $\langle$ proof $\rangle$

lemma zip-truncate-right $:$ zip xs ys $=$ zip xs (take (length xs) ys) $\langle$ proof $\rangle$

Lemmas zip-append1 and zip-append2 in theory HOL.List have unnecessary take (length -) in them. Here are replacements.

lemma zip-append-left :

$z i p(x s @ y s) z s=z i p x s$ zs @ zip ys (drop (length xs) zs)

$\langle$ proof $\rangle$

lemma zip-append-right :

zip xs $(y s @ z s)=z i p x s$ ys @ zip (drop (length ys) xs) zs 
$\langle$ proof $\rangle$

lemma length-concat-map-split-zip :

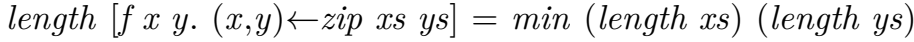

$\langle$ proof $\rangle$

lemma concat-map-split-eq-map-split-zip :

$[f x y .(x, y) \leftarrow z i p$ xs $y s]=\operatorname{map}($ case-prod $f)($ zip xs ys $)$

$\langle$ proof $\rangle$

lemma set-zip-map2 :

$(a, z) \in \operatorname{set}(z i p x s(\operatorname{map} f y s)) \Longrightarrow \exists b .(a, b) \in \operatorname{set}($ zip xs ys $) \wedge z=f b$ $\langle$ proof $\rangle$

\subsection{2 concat}

lemma concat-eq :

list-all2 ( $\lambda x s$ ys. length $x s=$ length ys) xss yss $\Longrightarrow$ concat $x s s=$ concat yss $\Longrightarrow x s s=y s s$

$\langle$ proof $\rangle$

lemma match-concat :

fixes bss:: 'b list list

defines eq-len: eq-len $\equiv \lambda x s$ ys. length $x s=$ length ys

shows $\forall$ as::'a list. length as $=$ length (concat bss)

$\langle$ proof $\rangle$

$\longrightarrow(\exists$ css::'a list list. as $=$ concat css $\wedge$ list-all2 eq-len css bss $)$

\subsection{3 strip-while}

lemma strip-while-0-nnil :

as $\neq[] \Longrightarrow$ set as $\neq 0 \Longrightarrow$ strip-while $((=) 0)$ as $\neq[]$

$\langle$ proof $\rangle$

\subsection{4 sum-list}

lemma const-sum-list :

$\forall x \in$ set $x s . f x=a \Longrightarrow$ sum-list $($ map $f x s)=a *$ (length $x s)$

$\langle$ proof $\rangle$

lemma sum-list-prod-cong :

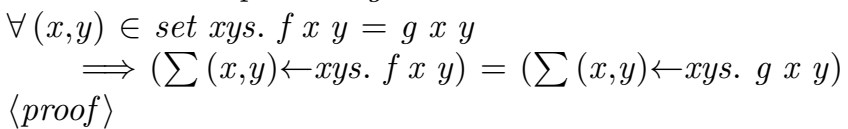

lemma sum-list-prod-map2 :

$\left(\sum(a, y) \leftarrow\right.$ zip as $($ map $f b s) . g$ a $\left.y\right)=\left(\sum(a, b) \leftarrow z i p\right.$ as bs. $g$ a $\left.(f b)\right)$ $\langle$ proof $\rangle$

lemma sum-list-fun-apply : $\left(\sum x \leftarrow x s . f x\right) y=\left(\sum x \leftarrow x s . f x y\right)$ 
$\langle$ proof $\rangle$

lemma sum-list-prod-fun-apply : $\left(\sum(x, y) \leftarrow x y s . f x\right.$ y $) z=\left(\sum(x, y) \leftarrow x y s . f x\right.$ y $\left.z\right)$ $\langle$ proof $\rangle$

lemma (in comm-monoid-add) sum-list-plus :

length $x s=$ length ys

$\langle$ proof $\rangle$

$\Longrightarrow$ sum-list $x s+$ sum-list ys $=$ sum-list $[a+b .(a, b) \leftarrow z i p x s$ ys $]$

lemma sum-list-const-mult-prod:

fixes $f::{ }^{\prime} a \Rightarrow{ }^{\prime} b \Rightarrow{ }^{\prime} r::$ semiring- 0

shows $r *\left(\sum(x, y) \leftarrow x y s . f x y\right)=\left(\sum(x, y) \leftarrow x y s . r *(f x y)\right)$

$\langle$ proof $\rangle$

lemma sum-list-mult-const-prod :

fixes $f::{ }^{\prime} a \Rightarrow{ }^{\prime} b \Rightarrow{ }^{\prime} r::$ semiring- 0

shows $\left(\sum(x, y) \leftarrow x y s . f x y\right) * r=\left(\sum(x, y) \leftarrow x y s .(f x y) * r\right)$

$\langle$ proof $\rangle$

lemma sum-list-update :

fixes $x s:: ' a:: a b$-group-add list

shows $n<$ length $x s \Longrightarrow$ sum-list $(x s[n:=y])=$ sum-list $x s-x s ! n+y$

$\langle$ proof $\rangle$

lemma sum-list-replicate0 : sum-list (replicate $n$ n 0$)=0$

$\langle$ proof $\rangle$

1.3.5 listset

lemma listset-ConsI $: x \in X \Longrightarrow x s \in$ listset $X s \Longrightarrow x \# x s \in$ listset $(X \# X s)$ $\langle$ proof $\rangle$

lemma listset-ConsD $: x \# x s \in$ listset $(A \# A s) \Longrightarrow x \in A \wedge x s \in$ listset $A s$ $\langle$ proof $\rangle$

lemma listset-Cons-conv :

$x s \in$ listset $(A \# A s) \Longrightarrow(\exists y$ ys. $y \in A \wedge y s \in$ listset $A s \wedge x s=y \# y s)$

$\langle$ proof $\rangle$

lemma listset-length $: x s \in$ listset $X s \Longrightarrow$ length $x s=$ length $X s$

$\langle$ proof $\rangle$

lemma set-sum-list-element :

$x \in\left(\sum A \leftarrow A s . A\right) \Longrightarrow \exists a s \in$ listset As. $x=\left(\sum a \leftarrow a s . a\right)$

$\langle$ proof $\rangle$

lemma set-sum-list-element-Cons :

assumes $x \in\left(\sum X \leftarrow(A \# A s) . X\right)$ 
shows $\exists a$ as. $a \in A \wedge$ as $\in$ listset As $\wedge x=a+\left(\sum b \leftarrow a s . b\right)$ $\langle$ proof $\rangle$

lemma sum-list-listset : as $\in$ listset $A s \Longrightarrow$ sum-list as $\in\left(\sum A \leftarrow A s . A\right)$ $\langle$ proof $\rangle$

lemma listsetI-nth :

length $x s=$ length $X s \Longrightarrow \forall n<$ length $x s . x s ! n \in X s ! n \Longrightarrow x s \in$ listset $X s$ $\langle$ proof $\rangle$

lemma listsetD-nth $: x s \in$ listset $X s \Longrightarrow \forall n<$ length $x s . x s ! n \in X s ! n$ $\langle$ proof $\rangle$

lemma set-listset-el-subset :

$x s \in$ listset $X s \Longrightarrow \forall X \in$ set $X s . X \subseteq A \Longrightarrow$ set $x s \subseteq A$ $\langle$ proof $\rangle$

\subsection{Functions}

\subsubsection{Miscellaneous facts}

lemma sum-fun-apply : finite $A \Longrightarrow\left(\sum a \in A . f a\right) x=\left(\sum a \in A . f a x\right)$ $\langle$ proof $\rangle$

lemma sum-single-nonzero :

finite $A \Longrightarrow(\forall x \in A . \forall y \in A$. $f x y=($ if $y=x$ then $g x$ else 0$)$ )

$\langle$ proof $\rangle$ $\Longrightarrow(\forall x \in A$. sum $(f x) A=g x)$

lemma distrib-comp-sum-right $:\left(T+T^{\prime}\right) \circ S=(T \circ S)+\left(T^{\prime} \circ S\right)$ $\langle$ proof $\rangle$

\subsubsection{Support of a function}

definition $\operatorname{supp}::\left({ }^{\prime} a \Rightarrow{ }^{\prime} b::\right.$ zero $) \Rightarrow{ }^{\prime} a$ set where supp $f=\{x$. $f x \neq 0\}$

lemma suppI: $f x \neq 0 \Longrightarrow x \in \operatorname{supp} f$ $\langle$ proof $\rangle$

lemma suppI-contra: $x \notin \operatorname{supp} f \Longrightarrow f x=0$ $\langle$ proof $\rangle$

lemma suppD: $x \in \operatorname{supp} f \Longrightarrow f x \neq 0$ $\langle$ proof $\rangle$

lemma suppD-contra: $f x=0 \Longrightarrow x \notin$ supp $f$ $\langle$ proof $\rangle$

lemma zerofun-imp-empty-supp : supp $0=\{\}$ $\langle$ proof $\rangle$ 


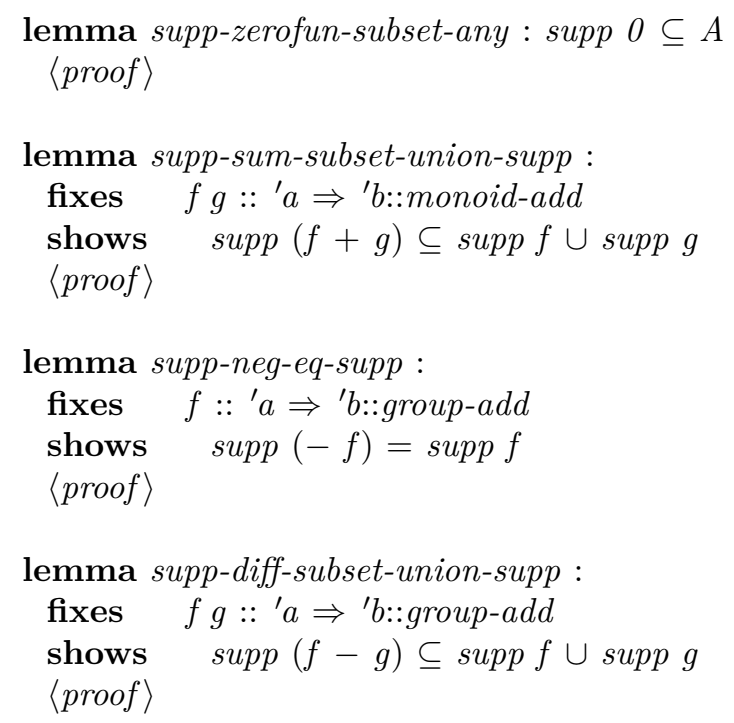

abbreviation restricto :: ('a $\Rightarrow^{\prime} b::$ zero $) \Rightarrow{ }^{\prime} a$ set $\Rightarrow\left({ }^{\prime} a \Rightarrow^{\prime} b\right)$ (infix $\downarrow$ 70) where restricto $f A \equiv(\lambda a$. if $a \in A$ then $f$ a else 0$)$

lemma supp-restricto : supp $(f \downarrow A) \subseteq A$

$\langle$ proof $\rangle$

lemma bij-betw-restricto : bij-betw $f A B \Longrightarrow$ bij-betw $(f \downarrow A) A B$ $\langle$ proof $\rangle$

\subsubsection{Convolution}

definition convolution ::

$\left({ }^{\prime} a::\right.$ group-add $\Rightarrow{ }^{\prime} b::\{$ comm-monoid-add,times $\left.\}\right) \Rightarrow\left({ }^{\prime} a \Rightarrow^{\prime} b\right) \Rightarrow\left({ }^{\prime} a \Rightarrow^{\prime} b\right)$ where convolution $f g$$$
=\left(\lambda x . \sum y \mid x-y \in \operatorname{supp} f \wedge y \in \operatorname{supp} g .(f(x-y)) * g y\right)
$$

- More often than not, this definition will be used in the case that ' $b$ is of class mult-zero, in which case the conditions $x-y \in \operatorname{supp} f$ and $y \in$ supp $g$ are obviously mathematically unnecessary. However, they also serve to ensure that the sum is taken over a finite set in the case that at least one of $f$ and $g$ is almost everywhere zero.

lemma convolution-zero :

fixes $\quad f g::{ }^{\prime} a::$ group- $a d d \Rightarrow{ }^{\prime} b::\{$ comm-monoid-add,mult-zero $\}$

shows $\quad f=0 \vee g=0 \Longrightarrow$ convolution $f g=0$

$\langle$ proof $\rangle$

lemma convolution-symm :

fixes $f g:$ ' $^{\prime}$ :: group-add $\Rightarrow{ }^{\prime} b::\{$ comm-monoid-add,times $\}$

shows convolution $f g$

$$
=\left(\lambda x . \sum y \mid y \in \operatorname{supp} f \wedge-y+x \in \operatorname{supp} g .(f y) * g(-y+x)\right)
$$


$\langle$ proof $\rangle$

lemma supp-convolution-subset-sum-supp :

fixes $f g:{ }^{\prime}$ ' $a::$ group-add $\Rightarrow ' b::\{$ comm-monoid-add,times $\}$

shows supp (convolution $f g) \subseteq \operatorname{supp~} f+\operatorname{supp} g$

$\langle$ proof $\rangle$

\subsection{Almost-everywhere-zero functions}

\subsubsection{Definition and basic properties}



lemma restrict-and-extend0-aezfun-is-aezfun :

assumes $f \in$ aezfun-set

shows $f \downarrow A \in$ aezfun-set

$\langle$ proof $\rangle$

\subsubsection{Delta (impulse) functions}

The notation is set up in the order output-input so that later when these are used to define the group ring $\mathrm{RG}$, it will be in order ring-element-groupelement.

definition deltafun :: 'b::zero $\Rightarrow{ }^{\prime} a \Rightarrow\left({ }^{\prime} a \Rightarrow{ }^{\prime} b\right)($ infix $\delta 70)$ where $b \delta a=(\lambda x$. if $x=a$ then $b$ else 0$)$ 


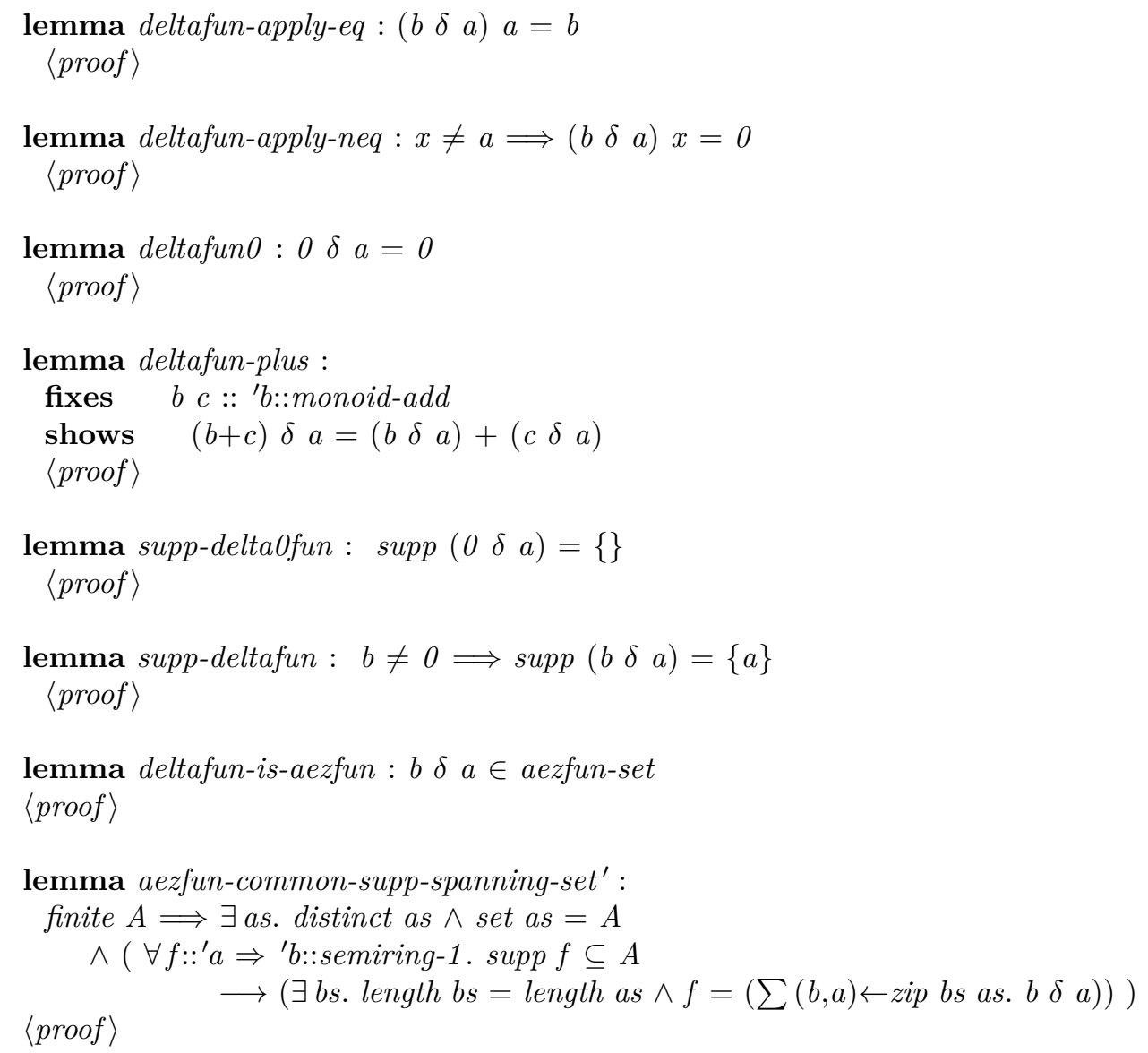

\subsubsection{Convolution of almost-everywhere-zero functions}

lemma convolution-eq-sum-over-supp-right :

fixes $g f::^{\prime} a::$ group-add $\Rightarrow{ }^{\prime} b::\{$ comm-monoid-add,mult-zero $\}$ assumes $g \in$ aezfun-set

shows convolution $f g=\left(\lambda x . \sum y \in \operatorname{supp} g .(f(x-y)) * g y\right)$ $\langle$ proof $\rangle$

lemma convolution-symm-eq-sum-over-supp-left :

fixes $f g:{ }^{\prime} a::$ group-add $\Rightarrow{ }^{\prime} b::\{$ comm-monoid-add,mult-zero $\}$ assumes $f \in$ aezfun-set

shows convolution $f g=\left(\lambda x . \sum y \in \operatorname{supp} f .(f y) * g(-y+x)\right)$ $\langle$ proof $\rangle$

lemma convolution-delta-left :

fixes $b:: ' b::\{c o m m-m o n o i d-a d d, m u l t-z e r o\}$

and $a::{ }^{\prime} a::$ group- $a d d$

and $f::{ }^{\prime} a \Rightarrow{ }^{\prime} b$

shows convolution $(b \delta a) f=(\lambda x . b * f(-a+x))$ 
$\langle$ proof $\rangle$

lemma convolution-delta-right :

fixes $b::{ }^{\prime} b::\{$ comm-monoid-add,mult-zero $\}$

and $f::{ }^{\prime} a::$ group- $a d d \Rightarrow{ }^{\prime} b$ and $a::^{\prime} a$

shows convolution $f(b \delta a)=(\lambda x . f(x-a) * b)$

$\langle$ proof $\rangle$

lemma convolution-delta-delta :

fixes $b 1$ b2 :: ' $b::\{$ comm-monoid-add,mult-zero $\}$

and a1 a2 :: 'a::group-add

shows convolution $(b 1 \delta a 1)(b 2 \delta a 2)=(b 1 * b 2) \delta(a 1+a 2)$

$\langle$ proof $\rangle$

lemma convolution-of-aezfun-is-aezfun :

fixes $\quad f g::{ }^{\prime} a::$ group-add $\Rightarrow ' b::\{$ comm-monoid-add,times $\}$

shows $\quad f \in$ aezfun-set $\Longrightarrow g \in$ aezfun-set $\Longrightarrow$ convolution $f g \in$ aezfun-set

$\langle$ proof $\rangle$

lemma convolution-assoc :

fixes $f h g::{ }^{\prime} a::$ group-add $\Rightarrow$ 'b::semiring-0

assumes f-aez: $f \in$ aezfun-set and $h$-aez: $h \in$ aezfun-set

shows convolution (convolution $f g) h=$ convolution $f$ (convolution $g h$ )

$\langle$ proof $\rangle$

lemma convolution-distrib-left :

fixes $g h f::{ }^{\prime} a::$ group-add $\Rightarrow$ 'b::semiring-0

assumes $g \in$ aezfun-set $h \in$ aezfun-set

shows convolution $f(g+h)=$ convolution $f g+$ convolution $f h$

$\langle$ proof $\rangle$

lemma convolution-distrib-right :

fixes $f g h::{ }^{\prime} a::$ group-add $\Rightarrow$ 'b::semiring-0

assumes $f \in$ aezfun-set $g \in$ aezfun-set

shows convolution $(f+g) h=$ convolution $f h+$ convolution $g h$

$\langle$ proof $\rangle$

\subsubsection{Type definition, instantiations, and instances}

typedef (overloaded) $\left({ }^{\prime} a:: z e r o, ' b\right)$ aezfun $=$ aezfun-set $::\left({ }^{\prime} b \Rightarrow^{\prime} a\right)$ set morphisms aezfun Abs-aezfun

$\langle$ proof $\rangle$

setup-lifting type-definition-aezfun

lemma aezfun-finite-supp : finite (supp (aezfun a))

$\langle$ proof $\rangle$

lemma aezfun-transfer : aezfun $a=$ aezfun $b \Longrightarrow a=b\langle$ proof $\rangle$ 
instantiation aezfun :: (zero, type) zero

begin

lift-definition zero-aezfun $::\left({ }^{\prime} a,,^{\prime} b\right)$ aezfun is $0::^{\prime} b \Rightarrow^{\prime} a$

$\langle$ proof $\rangle$

instance $\langle$ proof $\rangle$

end

lemma zero-aezfun-transfer : Abs-aezfun $\left(\left(0::^{\prime}\right.\right.$ b::zero $) \delta\left(0::^{\prime} a::\right.$ zero $\left.)\right)=0$

$\langle$ proof $\rangle$

lemma zero-aezfun-apply [simp]: aezfun $0 x=0$

$\langle$ proof $\rangle$

instantiation aezfun :: (monoid-add, type) plus

begin

lift-definition plus-aezfun :: ('a, 'b) aezfun $\Rightarrow\left({ }^{\prime} a,{ }^{\prime} b\right)$ aezfun $\Rightarrow\left({ }^{\prime} a,{ }^{\prime} b\right)$ aezfun is $\quad \lambda f g . f+g$

$\langle$ proof $\rangle$

instance $\langle$ proof $\rangle$

end

lemma plus-aezfun-apply $[$ simp $]$ : aezfun $(a+b) x=$ aezfun $a x+$ aezfun $b x$ $\langle$ proof $\rangle$

instance aezfun :: (monoid-add, type) semigroup-add $\langle$ proof $\rangle$

instance aezfun :: (monoid-add, type) monoid-add

$\langle$ proof $\rangle$

lemma sum-list-aezfun-apply [simp $]$ :

aezfun (sum-list as) $x=\left(\sum a \leftarrow\right.$ as. aezfun a $\left.x\right)$

$\langle$ proof $\rangle$

lemma sum-list-map-aezfun-apply [simp] :

aezfun $\left(\sum a \leftarrow a s . f a\right) x=\left(\sum a \leftarrow a s\right.$. aezfun $\left.(f a) x\right)$

$\langle$ proof $\rangle$

lemma sum-list-map-aezfun [simp] :

aezfun $\left(\sum a \leftarrow a s . f a\right)=\left(\sum a \leftarrow a s\right.$. aezfun $\left.(f a)\right)$

$\langle$ proof $\rangle$

lemma sum-list-prod-map-aezfun-apply :

aezfun $\left(\sum(x, y) \leftarrow x y s\right.$. $\left.f x y\right) a=\left(\sum(x, y) \leftarrow x y s\right.$. aezfun $\left.(f x y) a\right)$ $\langle$ proof $\rangle$

lemma sum-list-prod-map-aezfun :

aezfun $\left(\sum(x, y) \leftarrow x y s\right.$. $\left.f x y\right)=\left(\sum(x, y) \leftarrow x y s\right.$. aezfun $\left.(f x y)\right)$ 


$$
\langle\text { proof }\rangle
$$

instance aezfun :: (comm-monoid-add, type) comm-monoid-add $\langle$ proof $\rangle$

lemma sum-aezfun-apply [simp] :

finite $A \Longrightarrow$ aezfun $\left(\sum A\right) x=\left(\sum a \in A\right.$. aezfun a $\left.x\right)$

$\langle$ proof $\rangle$

instantiation aezfun :: (group-add, type) minus

begin

lift-definition minus-aezfun :: ('a, 'b) aezfun $\Rightarrow\left({ }^{\prime} a,{ }^{\prime} b\right)$ aezfun $\Rightarrow\left({ }^{\prime} a,{ }^{\prime} b\right)$ aezfun is $\quad \lambda f g . f-g$

$\langle$ proof $\rangle$

instance $\langle$ proof $\rangle$

end

lemma minus-aezfun-apply [simp]: aezfun $(a-b) x=$ aezfun $a x-$ aezfun $b x$ $\langle$ proof $\rangle$

instantiation aezfun :: (group-add, type) uminus

begin

lift-definition uminus-aezfun :: ('a, 'b) aezfun $\Rightarrow\left({ }^{\prime} a,{ }^{\prime} b\right)$ aezfun is $\lambda f .-f$

$\langle$ proof $\rangle$

instance $\langle$ proof $\rangle$

end

lemma uminus-aezfun-apply [simp]: aezfun $(-a) x=-$ aezfun a $x$ $\langle$ proof $\rangle$

lemma aezfun-left-minus $[$ simp $]$ :

fixes $a::\left({ }^{\prime} a::\right.$ group-add, 'b) aezfun

shows $-a+a=0$

$\langle$ proof $\rangle$

lemma aezfun-diff-minus [simp] :

fixes $a b::\left({ }^{\prime} a::\right.$ group-add, 'b) aezfun

shows $a-b=a+-b$

$\langle$ proof $\rangle$

instance aezfun :: (group-add, type) group-add

$\langle$ proof $\rangle$

instance aezfun :: (ab-group-add, type) ab-group-add $\langle$ proof $\rangle$

instantiation aezfun :: (\{one,zero $\}$, zero $)$ one

begin

lift-definition one-aezfun :: $\left({ }^{\prime} a,{ }^{\prime} b\right)$ aezfun is $1 \delta 0$ 




lemma one-aezfun-apply-eq [simp]: aezfun $10=1$ $\langle$ proof $\rangle$

lemma one-aezfun-apply-neq $[$ simp $]: x \neq 0 \Longrightarrow$ aezfun $1 x=0$ $\langle$ proof $\rangle$

instance aezfun :: (zero-neq-one, zero) zero-neq-one $\langle$ proof $\rangle$

instantiation aezfun :: (\{comm-monoid-add,times $\}$, group-add $)$ times begin

lift-definition times-aezfun :: ('a, 'b) aezfun $\Rightarrow\left({ }^{\prime} a,{ }^{\prime} b\right)$ aezfun $\Rightarrow\left({ }^{\prime} a,,^{\prime} b\right)$ aezfun is $\quad \lambda f g$. convolution $f g$ $\langle$ proof $\rangle$

instance $\langle$ proof $\rangle$

end

lemma convolution-transfer :

assumes $f \in$ aezfun-set $g \in$ aezfun-set

shows Abs-aezfun (convolution $f g$ ) $=$ Abs-aezfun $f *$ Abs-aezfun $g$ $\langle$ proof $\rangle$

instance aezfun :: (\{comm-monoid-add,mult-zero $\}$, group-add) mult-zero $\langle$ proof $\rangle$

instance aezfun :: (semiring-0, group-add) semiring-0

$\langle$ proof $\rangle$

instance aezfun :: (ring, group-add) ring $\langle$ proof $\rangle$

instance aezfun :: (\{semiring-0,monoid-mult,zero-neq-one $\}$, group-add) monoid-mult $\langle$ proof $\rangle$

instance aezfun :: (ring-1, group-add) ring-1 〈proof $\rangle$

\subsubsection{Transfer facts}

abbreviation aezdeltafun :: 'b::zero $\Rightarrow{ }^{\prime} a \Rightarrow\left({ }^{\prime} b,{ }^{\prime} a\right)$ aezfun (infix $\delta \delta 70$ ) where $b \delta \delta a \equiv A b s$-aezfun $\left(\begin{array}{lll}b & \delta & a\end{array}\right)$ 


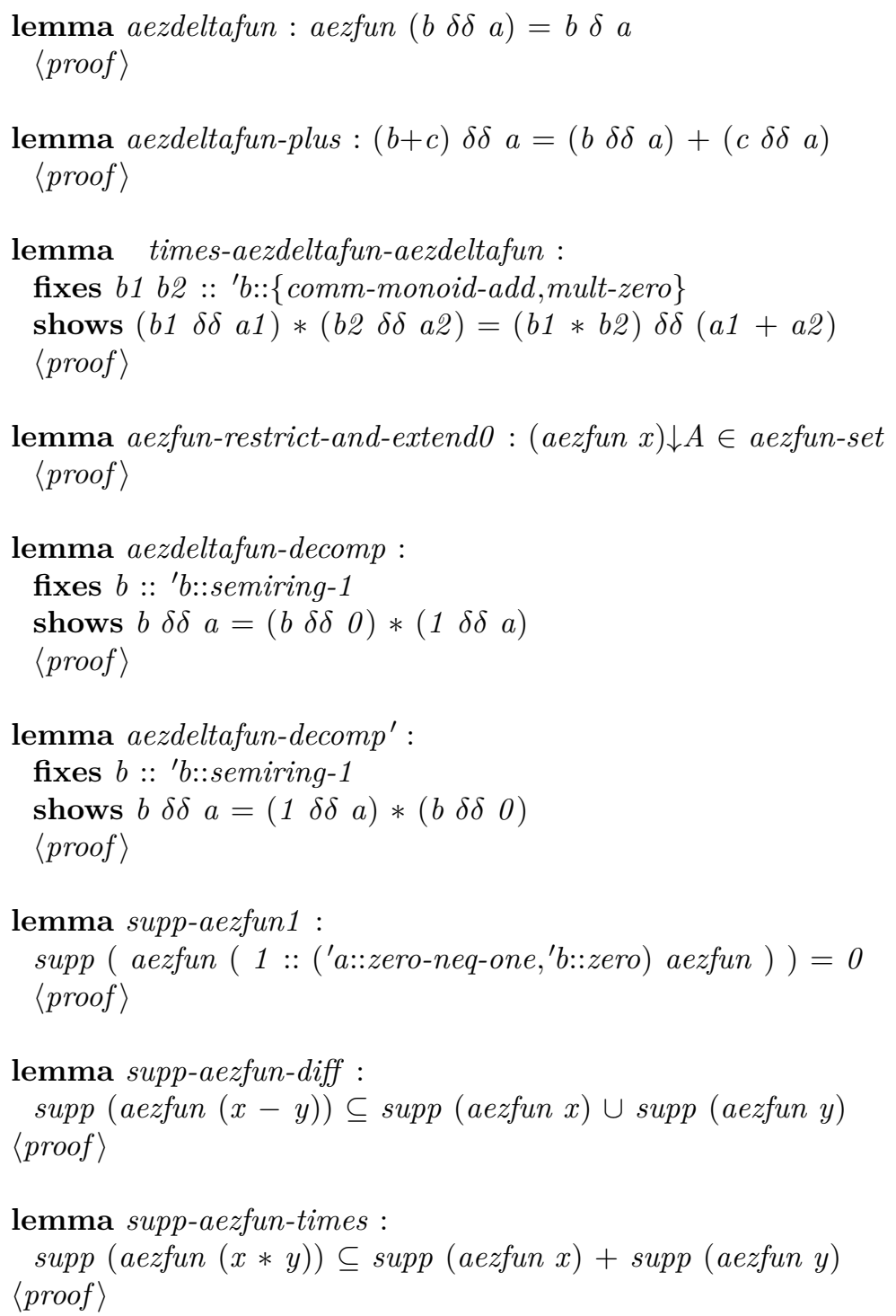

\subsubsection{Almost-everywhere-zero functions with constrained sup- port}

The name of the next definition anticipates aezfun-common-supp-spanning-set below.

\footnotetext{
definition aezfun-setspan :: 'a set $\Rightarrow\left({ }^{\prime} b:: z e r o,{ }^{\prime} a\right)$ aezfun set where aezfun-setspan $A=\{x$. supp $($ aezfun $x) \subseteq A\}$

lemma aezfun-setspanD : $x \in$ aezfun-setspan $A \Longrightarrow \operatorname{supp}($ aezfun $x) \subseteq A$ $\langle$ proof $\rangle$

lemma aezfun-setspanI : supp (aezfun $x) \subseteq A \Longrightarrow x \in$ aezfun-setspan $A$
} 
$\langle$ proof $\rangle$

lemma aezfun-common-supp-spanning-set :

assumes finite $A$

shows $\exists$ as. distinct as $\wedge$ set as $=A \wedge($

$\forall x::(' b::$ semiring-1,'a) aezfun $\in$ aezfun-setspan $A$.

$\exists$ bs. length bs = length as $\wedge x=\left(\sum(b, a) \leftarrow\right.$ zip bs as. $\left.b \delta \delta a\right)$

$\langle$ proof $\rangle$

lemma aezfun-common-supp-spanning-set-decomp :

fixes $G::{ }^{\prime} g:$ :group-add set

assumes finite $G$

shows $\exists$ gs. distinct gs $\wedge$ set gs $=G \wedge($

$\forall x::\left({ }^{\prime} r::\right.$ semiring- $\left.1,{ }^{\prime} g\right)$ aezfun $\in$ aezfun-setspan $G$.

$\exists$ rs. length $r s=$ length gs

$\wedge x=\left(\sum(r, g) \leftarrow z i p\right.$ rs gs. $\left.\left(\begin{array}{rll}r & \delta \delta & 0\end{array}\right) *\left(\begin{array}{lll}1 & \delta \delta & g\end{array}\right)\right)$

$\langle$ proof $\rangle$

lemma aezfun-decomp-aezdeltafun :

fixes $c::\left({ }^{\prime} r::\right.$ semiring-1,'a) aezfun

shows $\exists$ ras. set $($ map snd ras $)=\operatorname{supp}($ aezfun $c) \wedge c=\left(\sum(r, a) \leftarrow\right.$ ras. $\left.r \delta \delta a\right)$

$\langle$ proof $\rangle$

lemma aezfun-setspan-el-decomp-aezdeltafun :

fixes $c::\left({ }^{\prime} r::\right.$ semiring-1,'a) aezfun

shows $c \in$ aezfun-setspan $A$

$\langle$ proof $\rangle$

$\Longrightarrow \exists$ ras. set $($ map snd ras $) \subseteq A \wedge c=\left(\sum(r, a) \leftarrow\right.$ ras. $\left.r \delta \delta a\right)$

lemma aezdelta0fun-commutes' :

fixes b1 b2 :: 'b::comm-semiring-1

shows $b 1 \delta \delta a *\left(\begin{array}{lll}b 2 & \delta \delta & 0\end{array}\right)=b 2 \delta \delta 0 *\left(\begin{array}{llll}b 1 & \delta \delta & a\end{array}\right)$

$\langle$ proof $\rangle$

lemma aezdelta0fun-commutes :

fixes $b:: ' b::$ comm-semiring-1

shows $c *(b \delta \delta 0)=b \delta \delta 0 * c$

$\langle$ proof $\rangle$

The following definition constrains the support of arbitrary almost-everywherezero functions, as a sort of projection onto a aezfun-setspan.

definition aezfun-setspan-proj :: 'a set $\Rightarrow\left({ }^{\prime} b:: z e r o,{ }^{\prime} a\right)$ aezfun $\Rightarrow\left({ }^{\prime} b:: z e r o,{ }^{\prime} a\right)$ aezfun where aezfun-setspan-proj $A x \equiv$ Abs-aezfun $(($ aezfun $x) \downarrow A)$

lemma aezfun-setspan-projD1 :

$a \in A \Longrightarrow$ aezfun (aezfun-setspan-proj $A x$ ) $a=$ aezfun $x a$

$\langle$ proof $\rangle$ 
lemma aezfun-setspan-projD2 :

$a \notin A \Longrightarrow$ aezfun (aezfun-setspan-proj $A x) a=0$

$\langle$ proof $\rangle$

lemma aezfun-setspan-proj-in-setspan :

aezfun-setspan-proj $A x \in$ aezfun-setspan $A$

$\langle$ proof $\rangle$

lemma aezfun-setspan-proj-zero : aezfun-setspan-proj $A 0=0$

$\langle$ proof $\rangle$

lemma aezfun-setspan-proj-aezdeltafun :

aezfun-setspan-proj $A(b \delta \delta a)=($ if $a \in A$ then $b \delta \delta$ a else 0$)$

$\langle$ proof $\rangle$

lemma aezfun-setspan-proj-add :

aezfun-setspan-proj $A(x+y)$

= aezfun-setspan-proj $A x+$ aezfun-setspan-proj $A y$

$\langle$ proof $\rangle$

lemma aezfun-setspan-proj-sum-list :

aezfun-setspan-proj $A\left(\sum x \leftarrow x s . f x\right)=\left(\sum x \leftarrow x s\right.$. aezfun-setspan-proj $\left.A(f x)\right)$ $\langle$ proof $\rangle$

lemma aezfun-setspan-proj-sum-list-prod :

aezfun-setspan-proj $A\left(\sum(x, y) \leftarrow x y s\right.$. $\left.f x y\right)$

$\langle$ proof $\rangle$

$$
=\left(\sum(x, y) \leftarrow x y s . \text { aezfun-setspan-proj } A(f x y)\right)
$$

\subsection{Polynomials}

lemma nonzero-coeffs-nonzero-poly : as $\neq[] \Longrightarrow$ set as $\neq 0 \Longrightarrow$ Poly as $\neq 0$ $\langle$ proof $\rangle$

lemma const-poly-nonzero-coeff :

assumes degree $p=0 p \neq 0$

shows coeff $p 0 \neq 0$

$\langle$ proof $\rangle$

lemma $p$ Cons-induct2 [case-names 00 lpCons rpCons pCons2]:

assumes 00: $P 00$

and lpCons: $\bigwedge a p . a \neq 0 \vee p \neq 0 \Longrightarrow P($ C Cons $a p) 0$

and $\quad r p$ Cons: $\bigwedge b q . b \neq 0 \vee q \neq 0 \Longrightarrow P 0($ Cons $b q)$

and $\quad$ Cons2: $\bigwedge a p b q . a \neq 0 \vee p \neq 0 \Longrightarrow b \neq 0 \vee q \neq 0 \Longrightarrow P p q$

shows $P p q$

$\Longrightarrow P(p$ Cons a $p)($ Cons $b q)$

$\langle$ proof $\rangle$ 


\subsection{Algebra of sets}

\subsubsection{General facts}

lemma zeroset-eqI: $0 \in A \Longrightarrow(\bigwedge a . a \in A \Longrightarrow a=0) \Longrightarrow A=0$ $\langle$ proof $\rangle$

lemma sum-list-sets-single $:\left(\sum X \leftarrow[A] . X\right)=A$ $\langle$ proof $\rangle$

lemma sum-list-sets-double $:\left(\sum X \leftarrow[A, B] . X\right)=A+B$ $\langle$ proof $\rangle$

\subsubsection{Additive independence of sets}

primrec add-independentS :: 'a::monoid-add set list $\Rightarrow$ bool where add-independent $S[]=$ True

| add-independent $S(A \# A s)=($ add-independent $S$ As

$$
\left.\wedge\left(\forall x \in\left(\sum B \leftarrow \text { As. } B\right) . \forall a \in A . a+x=0 \longrightarrow a=0\right)\right)
$$

lemma add-independentS-doubleI:

assumes $\bigwedge b a . b \in B \Longrightarrow a \in A \Longrightarrow a+b=0 \Longrightarrow a=0$

shows add-independent $S[A, B]$

$\langle$ proof $\rangle$

lemma add-independentS-doubleD:

assumes add-independent $S[A, B]$

shows $\bigwedge b a . b \in B \Longrightarrow a \in A \Longrightarrow a+b=0 \Longrightarrow a=0$

$\langle$ proof $\rangle$

lemma add-independentS-double-iff :

$a d d$-independent $S[A, B]=(\forall b \in B . \forall a \in A . a+b=0 \longrightarrow a=0)$

$\langle$ proof $\rangle$

lemma add-independentS-Cons-conv-sum-right :

add-independent $S(A \# A s)$ $\langle$ proof $\rangle$

$=\left(\right.$ add-independent $S\left[A, \sum B \leftarrow A s . B\right] \wedge$ add-independent $\left.S A s\right)$

lemma add-independentS-double-sum-conv-append :

$\llbracket \forall X \in$ set As. $O \in X ;$ add-independent $S$ As; add-independentS Bs;

add-independent $S\left[\sum X \leftarrow A s . X, \sum X \leftarrow B s . X\right] \rrbracket$

$\langle$ proof $\rangle$

$\Longrightarrow$ add-independent $S(A s @ B s)$

lemma add-independentS-ConsI :

assumes add-independent $S$ As

$\bigwedge x a . \llbracket x \in\left(\sum X \leftarrow A s . X\right) ; a \in A ; a+x=0 \rrbracket \Longrightarrow a=0$

shows add-independent $S(A \# A s)$

$\langle$ proof $\rangle$ 
lemma add-independentS-append-reduce-right :

add-independentS $(A s @ B s) \Longrightarrow$ add-independent $S B s$

$\langle$ proof $\rangle$

lemma add-independentS-append-reduce-left :

add-independent $(A s @ B s) \Longrightarrow 0 \in\left(\sum X \leftarrow B s . X\right) \Longrightarrow$ add-independent $S A s$ $\langle$ proof $\rangle$

lemma add-independentS-append-conv-double-sum :

add-independent $S(A s @ B s) \Longrightarrow$ add-independent $S\left[\sum X \leftarrow A s . X, \sum X \leftarrow B s . X\right]$ $\langle$ proof $\rangle$

\subsubsection{Inner direct sums}

definition inner-dirsum :: 'a::monoid-add set list $\Rightarrow$ 'a set

where inner-dirsum $A s=\left(\right.$ if add-independentS As then $\sum A \leftarrow A s . A$ else 0$)$

Some syntactic sugar for inner-dirsum, borrowed from theory HOL.List.

syntax

-inner-dirsum :: pttrn $=>{ }^{\prime} a$ list $=>{ }^{\prime} b=>$ 'b

$((3 \bigoplus-\leftarrow$-. - $)[0,51,10] 10)$

translations - Beware of argument permutation!

$\bigoplus M \leftarrow M s . b==$ CONST inner-dirsum (CONST map $(\% M . b) M s)$

abbreviation inner-dirsum-double ::

'a: :monoid-add set $\Rightarrow{ }^{\prime} a$ set $\Rightarrow$ 'a set (infixr $\left.\oplus 70\right)$

where inner-dirsum-double $A B \equiv$ inner-dirsum $[A, B]$

lemma inner-dirsumI :

$M=\left(\sum N \leftarrow N s . N\right) \Longrightarrow$ add-independent $S s \Longrightarrow M=(\bigoplus N \leftarrow N s . N)$

$\langle$ proof $\rangle$

lemma inner-dirsum-doubleI :

$M=A+B \Longrightarrow$ add-independent $[A, B] \Longrightarrow M=A \oplus B$

$\langle$ proof $\rangle$

lemma inner-dirsumD :

add-independent $S \mathrm{Ms} \Longrightarrow(\bigoplus M \leftarrow M s . M)=\left(\sum M \leftarrow M s . M\right)$

$\langle$ proof $\rangle$

lemma inner-dirsumD2 : ᄀadd-independent $S M s \Longrightarrow(\bigoplus M \leftarrow M s . M)=0$ $\langle$ proof $\rangle$

lemma inner-dirsum-Nil : $(\bigoplus A \leftarrow[] . A)=0$

$\langle$ proof $\rangle$

lemma inner-dirsum-single $D:(\bigoplus N \leftarrow[M] . N)=M$

$\langle$ proof $\rangle$ 


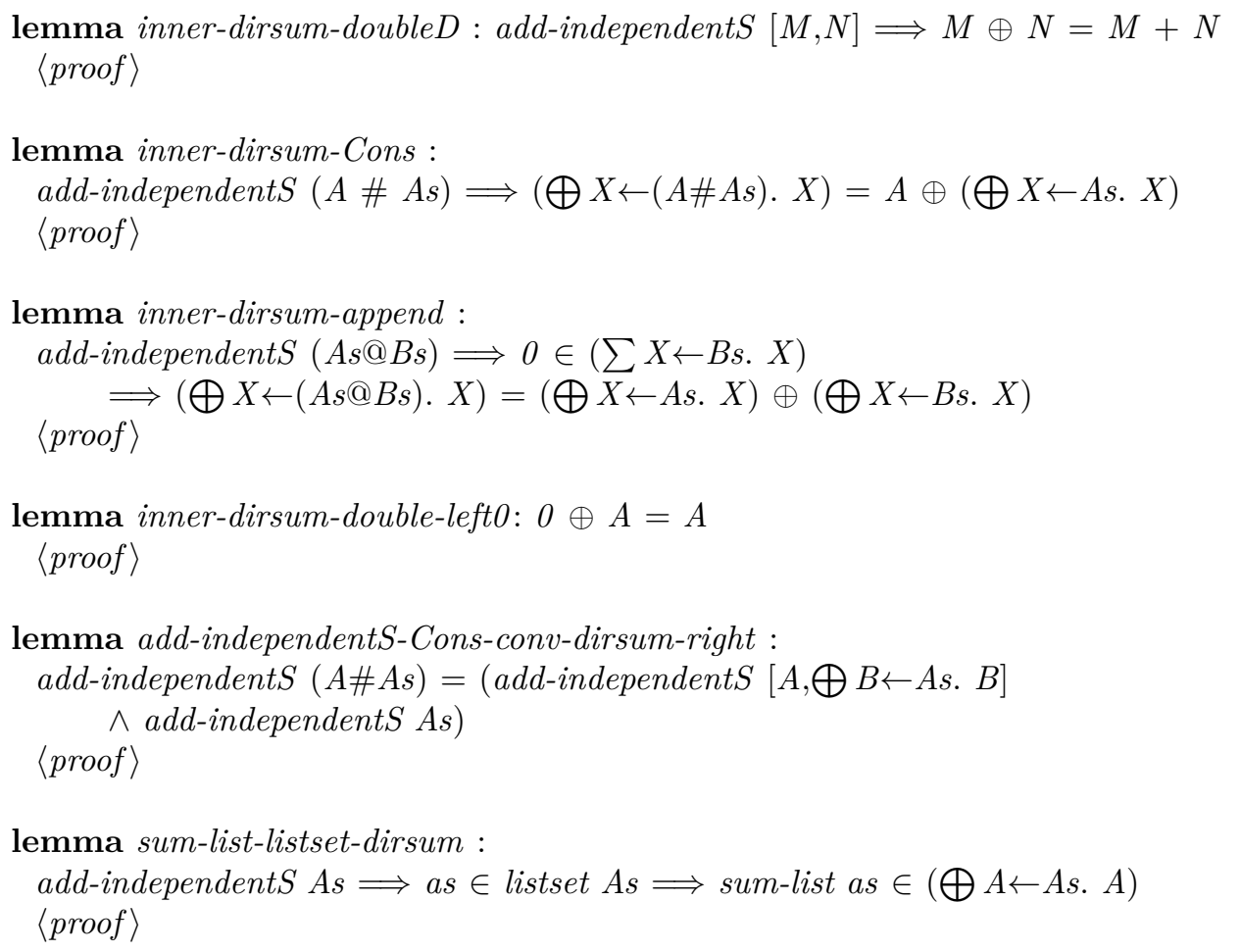

\section{Groups}

\section{$2.1 \quad$ Locales and basic facts}

\subsubsection{Locale Group and finite variant FinGroup}

Define a Group to be a closed subset of $U N I V$ for the group-add class.

locale Group =

fixes $G::{ }^{\prime} g:$ :group-add set

assumes nonempty : $G \neq\{\}$

and diff-closed: $\bigwedge g h . g \in G \Longrightarrow h \in G \Longrightarrow g-h \in G$

lemma trivial-Group : Group 0 $\langle$ proof $\rangle$

locale FinGroup = Group $G$

for $G::{ }^{\prime} g:$ :group-add set

+ assumes finite: finite $G$

lemma (in FinGroup) Group : Group G〈proof $\rangle$

lemma (in Group) FinGroupI : finite G $G$ FinGroup $G\langle$ proof $\rangle$

context Group 
begin

abbreviation Subgroup ::

'g set $\Rightarrow$ bool where Subgroup $H \equiv$ Group $H \wedge H \subseteq G$

lemma SubgroupD1 : Subgroup $H \Longrightarrow$ Group $H\langle$ proof $\rangle$

lemma zero-closed : $0 \in G$

$\langle$ proof $\rangle$

lemma obtain-nonzero: assumes $G \neq 0$ obtains $g$ where $g \in G$ and $g \neq 0$ $\langle$ proof $\rangle$

lemma zeroS-closed : $0 \subseteq G$

$\langle$ proof $\rangle$

lemma neg-closed $: g \in G \Longrightarrow-g \in G$

$\langle$ proof $\rangle$

lemma add-closed $: g \in G \Longrightarrow h \in G \Longrightarrow g+h \in G$

$\langle$ proof $\rangle$

lemma neg-add-closed $: g \in G \Longrightarrow h \in G \Longrightarrow-g+h \in G$

$\langle$ proof $\rangle$

lemma sum-list-closed : set (map fas) $\subseteq G \Longrightarrow\left(\sum a \leftarrow a s . f a\right) \in G$ $\langle$ proof $\rangle$

lemma sum-list-closed-prod :

set $($ map $($ case-prod $f) x y s) \subseteq G \Longrightarrow\left(\sum(x, y) \leftarrow x y s\right.$. $\left.f x y\right) \in G$ $\langle$ proof $\rangle$

lemma set-plus-closed : $A \subseteq G \Longrightarrow B \subseteq G \Longrightarrow A+B \subseteq G$ $\langle$ proof $\rangle$

lemma zip-add-closed :

set as $\subseteq G \Longrightarrow$ set bs $\subseteq G \Longrightarrow$ set $[a+b .(a, b) \leftarrow z i p$ as $b s] \subseteq G$ $\langle$ proof $\rangle$

lemma list-diff-closed :

set $g s \subseteq G \Longrightarrow$ set $h s \subseteq G \Longrightarrow$ set $[x-y .(x, y) \leftarrow z i p$ gs $h s] \subseteq G$ $\langle$ proof $\rangle$

lemma add-closed-converse-right $: g+x \in G \Longrightarrow g \in G \Longrightarrow x \in G$ $\langle$ proof $\rangle$

lemma add-closed-inverse-right : $x \notin G \Longrightarrow g \in G \Longrightarrow g+x \notin G$ $\langle$ proof $\rangle$ 
lemma add-closed-converse-left $: g+x \in G \Longrightarrow x \in G \Longrightarrow g \in G$ $\langle$ proof $\rangle$

lemma add-closed-inverse-left : $g \notin G \Longrightarrow x \in G \Longrightarrow g+x \notin G$ $\langle$ proof $\rangle$

lemma right-translate-bij : assumes $g \in G$

shows bij-betw $(\lambda x . x+g) G G$ $\langle$ proof $\rangle$

lemma right-translate-sum $: g \in G \Longrightarrow\left(\sum h \in G . f h\right)=\left(\sum h \in G . f(h+g)\right)$ $\langle$ proof $\rangle$

end

\subsubsection{Abelian variant locale AbGroup}

locale $A b G r o u p=$ Group $G$

for $G::{ }^{\prime} g:: a b$-group-add set

begin

lemmas nonempty = nonempty

lemmas zero-closed $=$ zero-closed

lemmas diff-closed $=$ diff-closed

lemmas add-closed $=$ add-closed

lemmas neg-closed $=$ neg-closed

lemma sum-closed : finite $A \Longrightarrow f^{\prime} A \subseteq G \Longrightarrow\left(\sum a \in A . f a\right) \in G$

$\langle$ proof $\rangle$

lemma subset-plus-right : $A \subseteq G+A$

$\langle$ proof $\rangle$

lemma subset-plus-left : $A \subseteq A+G$

$\langle$ proof $\rangle$

end

\section{$2.2 \quad$ Right cosets}

context Group

begin

definition rcoset-rel :: 'g set $\Rightarrow\left({ }^{\prime} g \times{ }^{\prime} g\right)$ set where rcoset-rel $H \equiv\left\{\left(g, g^{\prime}\right) . g \in G \wedge g^{\prime} \in G \wedge g-g^{\prime} \in H\right\}$

lemma (in Group) rcosets :

assumes subgrp: Subgroup $H$ and $g: g \in G$

shows $\quad($ rcoset-rel $H) "\{g\}=H+\{g\}$ 
$\langle$ proof $\rangle$

lemma rcoset-equiv :

assumes Subgroup $H$

shows equiv $G$ (rcoset-rel $H)$

$\langle$ proof $\rangle$

lemma rcoset 0 : Subgroup $H \Longrightarrow($ rcoset-rel $H) "\{0\}=H$

$\langle$ proof $\rangle$

definition is-rcoset-replist :: 'g set $\Rightarrow{ }^{\prime} g$ list $\Rightarrow$ bool

where is-rcoset-replist $H$ gs

$$
\begin{aligned}
\equiv \text { set } g s \subseteq G \wedge \text { distinct }(\operatorname{map}(\lambda g .(\text { rcoset-rel } H) "\{g\}) g s) \\
\wedge G=(\bigcup g \in \text { set gs. (rcoset-rel } H) "\{g\})
\end{aligned}
$$

lemma is-rcoset-replistD-set : is-rcoset-replist $H$ gs $\Longrightarrow$ set $g s \subseteq G$ $\langle$ proof $\rangle$

lemma is-rcoset-replistD-distinct :

is-rcoset-replist H gs $\Longrightarrow$ distinct (map $(\lambda g$. (rcoset-rel $\left.\left.H){ }^{\prime \prime}\{g\}\right) g s\right)$

$\langle$ proof $\rangle$

lemma is-rcoset-replistD-cosets :

is-rcoset-replist $H$ gs $\Longrightarrow G=(\bigcup g \in$ set gs. (rcoset-rel $\left.H){ }^{\prime}\{g\}\right)$

$\langle$ proof $\rangle$

lemma group-eq-subgrp-rcoset-un :

Subgroup $H \Longrightarrow$ is-rcoset-replist $H$ gs $\Longrightarrow G=(\bigcup g \in$ set gs. $H+\{g\})$

$\langle$ proof $\rangle$

lemma is-rcoset-replist-imp-nrelated-nth :

assumes Subgroup $H$ is-rcoset-replist $H$ gs

shows $\bigwedge i j . i<$ length $g s \Longrightarrow j<$ length $g s \Longrightarrow i \neq j \Longrightarrow g s ! i-g s ! j \notin H$ $\langle$ proof $\rangle$

lemma is-rcoset-replist-Cons :

is-rcoset-replist $H$ ( $g \# g s) \longleftrightarrow$

$g \in G \wedge$ set $g s \subseteq G$

$\wedge($ rcoset-rel $H) "$ " $\{g\} \notin$ set $(\operatorname{map}(\lambda x$. (rcoset-rel $H) "$ " $\{x\}) g s)$

$\wedge$ distinct $(\operatorname{map}(\lambda x$. (rcoset-rel $H) "\{x\})$ gs)

$\wedge G=($ rcoset-rel $H) "\{g\} \cup(\bigcup x \in$ set gs. (rcoset-rel $H) "\{x\})$

$\langle$ proof $\rangle$

lemma rcoset-replist-Hrep :

assumes Subgroup $H$ is-rcoset-replist $H$ gs

shows $\exists g \in$ set gs. $g \in H$

$\langle$ proof $\rangle$

lemma rcoset-replist-reorder : 
is-rcoset-replist $H\left(g s @ g \# g s^{\prime}\right) \Longrightarrow$ is-rcoset-replist $H\left(g \# g s @ g s^{\prime}\right)$ $\langle$ proof $\rangle$

lemma rcoset-replist-replacehd:

assumes Subgroup $H g^{\prime} \in($ rcoset-rel $H)$ " $\{g\}$ is-rcoset-replist $H(g \# g s)$

shows is-rcoset-replist $H\left(g^{\prime} \# g s\right)$

$\langle$ proof $\rangle$

end

lemma (in FinGroup) ex-rcoset-replist :

assumes Subgroup H

shows $\exists$ gs. is-rcoset-replist $H$ gs

$\langle$ proof $\rangle$

lemma (in FinGroup) ex-rcoset-replist-hdo :

assumes Subgroup $H$

shows $\exists$ gs. is-rcoset-replist $H(0 \# g s)$

$\langle$ proof $\rangle$

\subsection{Group homomorphisms}

\subsubsection{Preliminaries}



lemma ker-im-iff : $(A \neq\{\} \wedge A \subseteq \operatorname{ker} f)=\left(f^{\prime} A=0\right)$ $\langle$ proof $\rangle$

\subsubsection{Locales}

The supp condition is not strictly necessary, but helps with equality and uniqueness arguments.

locale GroupHom = Group $G$

for $G::{ }^{\prime} g::$ group-add set

+ fixes $T::{ }^{\prime} g \Rightarrow{ }^{\prime} h::$ group-add

assumes hom : $\bigwedge g g^{\prime} . g \in G \Longrightarrow g^{\prime} \in G \Longrightarrow T\left(g+g^{\prime}\right)=T g+T g^{\prime}$

and supp: supp $T \subseteq G$

abbreviation (in GroupHom) Ker $\equiv$ ker $T \cap G$

abbreviation (in GroupHom) $\operatorname{Im} G \equiv T^{\prime} G$ 
locale GroupEnd = GroupHom $G T$

for $G::{ }^{\prime} g:$ :group-add set

and $T::{ }^{\prime} g \Rightarrow{ }^{\prime} g$

+ assumes endomorph: $\operatorname{Im} G \subseteq G$

locale GroupIso $=$ GroupHom $G T$

for $G::{ }^{\prime} g::$ group-add set

and $T::{ }^{\prime} g \Rightarrow{ }^{\prime} h::$ group-add

+ fixes $H:: ' h$ set

assumes bijective: bij-betw $T G H$

\subsubsection{Basic facts}

lemma (in Group) trivial-GroupHom : GroupHom $G\left(0::\left({ }^{\prime} g \Rightarrow{ }^{\prime} h::\right.\right.$ group-add $\left.)\right)$ $\langle$ proof $\rangle$

lemma (in Group) GroupHom-idhom : GroupHom $G(i d \downarrow G)$

$\langle$ proof $\rangle$

context GroupHom

begin

lemma im-zero : $T 0=0$

$\langle$ proof $\rangle$

lemma zero-in-Ker : $0 \in$ Ker

$\langle$ proof $\rangle$

lemma comp-zero : $T \circ 0=0$

$\langle$ proof $\rangle$

lemma im-neg : $T(-g)=-T g$

$\langle$ proof $\rangle$

lemma im-diff $: g \in G \Longrightarrow g^{\prime} \in G \Longrightarrow T\left(g-g^{\prime}\right)=T g-T g^{\prime}$ $\langle$ proof $\rangle$

lemma eq-im-imp-diff-in-Ker $: \llbracket g \in G ; h \in G ; T g=T h \rrbracket \Longrightarrow g-h \in$ Ker $\langle$ proof $\rangle$

lemma im-sum-list-prod :

set (map (case-prod $f)$ xys $) \subseteq G$

$\langle$ proof $\rangle$

$\Longrightarrow T\left(\sum(x, y) \leftarrow x y s . f x y\right)=\left(\sum(x, y) \leftarrow x y s . T(f x y)\right)$

lemma distrib-comp-sum-left :

range $S \subseteq G \Longrightarrow$ range $S^{\prime} \subseteq G \Longrightarrow T \circ\left(S+S^{\prime}\right)=(T \circ S)+\left(T \circ S^{\prime}\right)$

$\langle$ proof $\rangle$ 


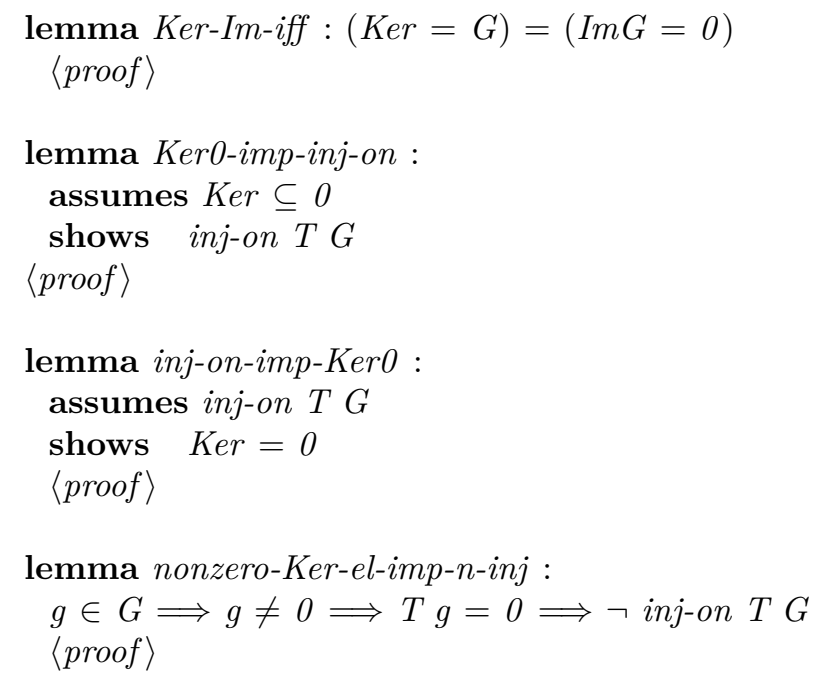

lemma Group-Ker : Group Ker

$\langle$ proof $\rangle$

lemma Group-Im : Group ImG

$\langle$ proof $\rangle$

lemma GroupHom-restrict0-subgroup : assumes Subgroup $H$ shows GroupHom $H(T \downarrow H)$ $\langle$ proof $\rangle$

lemma im-subgroup : assumes Subgroup $H$ shows Group.Subgroup $\operatorname{Im} G(T \cdot H)$ $\langle$ proof $\rangle$

lemma GroupHom-composite-left : assumes $\operatorname{Im} G \subseteq H$ GroupHom $H S$ shows GroupHom $G(S \circ T)$ $\langle$ proof $\rangle$

lemma $i d h o m$-left : $T$ ' $G \subseteq H \Longrightarrow(i d \downarrow H) \circ T=T$ $\langle$ proof $\rangle$

end

2.3.4 Basic facts about endomorphisms

context GroupEnd

begin

lemmas hom $=$ hom 
lemma range : range $T \subseteq G$

$\langle$ proof $\rangle$

lemma proj-decomp :

assumes $\bigwedge g . g \in G \Longrightarrow T(T g)=T g$

shows $G=\operatorname{Ker} \oplus \operatorname{Im} G$

$\langle$ proof $\rangle$

end

\subsubsection{Basic facts about isomorphisms}

context GroupIso

begin

abbreviation $i n v T \equiv($ the-inv-into $G T) \downarrow H$

lemma $\operatorname{Im} G: \operatorname{Im} G=H\langle$ proof $\rangle$

lemma GroupH : Group $H\langle$ proof $\rangle$

lemma invT-onto : invT' $H=G$

$\langle$ proof $\rangle$

lemma inj-on-invT : inj-on invT $H$

$\langle$ proof $\rangle$

lemma bijective-invT : bij-betw invT $H G$

$\langle$ proof $\rangle$

lemma invT-into $: h \in H \Longrightarrow$ invT $h \in G$

$\langle$ proof $\rangle$

lemma $T$-invT $: h \in H \Longrightarrow T($ inv $T h)=h$

$\langle$ proof $\rangle$

lemma invT-eq: $g \in G \Longrightarrow T g=h \Longrightarrow i n v T h=g$

$\langle$ proof $\rangle$

lemma inv : GroupIso H invT G

$\langle$ proof $\rangle$

end

\subsubsection{Hom-sets}

definition GroupHomSet :: 'g::group-add set $\Rightarrow$ ' $h::$ group-add set $\Rightarrow\left({ }^{\prime} g \Rightarrow{ }^{\prime} h\right)$ set where GroupHomSet $G H \equiv\{T$. GroupHom $G T\} \cap\{T$. T' $G \subseteq H\}$ 
lemma GroupHomSetI :

GroupHom $G T \Longrightarrow T$ ' $G \subseteq H \Longrightarrow T \in$ GroupHomSet $G H$

$\langle$ proof $\rangle$

lemma GroupHomSetD-GroupHom :

$T \in$ GroupHomSet $G \mathrm{H} \Longrightarrow$ GroupHom $G T$

$\langle$ proof $\rangle$

lemma GroupHomSetD-Im : $T \in$ GroupHomSet $G H \Longrightarrow T$ ' $G \subseteq H$

$\langle$ proof $\rangle$

lemma (in Group) Group-GroupHomSet :

fixes $H:: ' h:: a b$-group-add set

assumes AbGroup $H$

shows Group (GroupHomSet G H)

$\langle$ proof $\rangle$

\subsection{Facts about collections of groups}

lemma listset-Group-plus-closed :

$\llbracket \forall G \in$ set Gs. Group $G$; as $\in$ listset $G s ;$ bs $\in$ listset $G s \rrbracket$

$\langle$ proof $\rangle$

$\Longrightarrow[a+b .(a, b) \leftarrow$ zip as $b s] \in$ listset Gs

lemma AbGroup-set-plus :

assumes AbGroup H AbGroup $G$

shows AbGroup $(H+G)$

$\langle$ proof $\rangle$

lemma AbGroup-sum-list :

$(\forall G \in$ set Gs. AbGroup $G) \Longrightarrow$ AbGroup $\left(\sum G \leftarrow G s . G\right)$

$\langle$ proof $\rangle$

lemma AbGroup-subset-sum-list :

$\forall G \in$ set Gs. AbGroup $G \Longrightarrow H \in$ set $G s \Longrightarrow H \subseteq\left(\sum G \leftarrow G s . G\right)$

$\langle$ proof $\rangle$

lemma independent-AbGroups-pairwise-int0 :

$\llbracket \forall G \in$ set Gs. AbGroup $G ;$ add-independent $S$ Gs; $G \in$ set $G s ; G^{\prime} \in$ set $G$ s;

$$
G \neq G^{\prime} \rrbracket \Longrightarrow G \cap G^{\prime}=0
$$

$\langle$ proof $\rangle$

lemma independent-AbGroups-pairwise-int0-double :

assumes AbGroup G AbGroup $G^{\prime}$ add-independent $\left[G, G^{\prime}\right]$

shows $G \cap G^{\prime}=0$

$\langle$ proof $\rangle$ 


\subsection{Inner direct sums of Abelian groups}

\subsubsection{General facts}

lemma AbGroup-inner-dirsum :

$\forall G \in$ set Gs. AbGroup $G \Longrightarrow$ AbGroup $(\bigoplus G \leftarrow G s . G)$

$\langle$ proof $\rangle$

lemma inner-dirsum-double-leftfull-imp-right0:

assumes Group $A B \neq\{\} A=A \oplus B$

shows $B=0$

$\langle$ proof $\rangle$

lemma AbGroup-subset-inner-dirsum :

$\llbracket \forall G \in$ set Gs. AbGroup $G ;$ add-independent $S$ Gs; $H \in$ set $G s \rrbracket$

$\langle$ proof $\rangle$

$\Longrightarrow H \subseteq(\bigoplus G \leftarrow G s . G)$

lemma AbGroup-nth-subset-inner-dirsum :

$\llbracket \forall G \in$ set Gs. AbGroup $G ;$ add-independentS $G s ; n<$ length $G s \rrbracket$

$\langle$ proof $\rangle$

$\Longrightarrow G s ! n \subseteq(\bigoplus G \leftarrow G s . G)$

lemma AbGroup-inner-dirsum-el-decomp-ex1-double :

assumes AbGroup $G$ AbGroup $H$ add-independent $S[G, H] x \in G \oplus H$

shows $\exists$ !gh. fst $g h \in G \wedge$ snd $g h \in H \wedge x=$ fst $g h+$ snd $g h$

$\langle$ proof $\rangle$

lemma AbGroup-inner-dirsum-el-decomp-ex1 :

$\llbracket \forall G \in$ set Gs. AbGroup $G$; add-independent $S$ Gs 》

$\Longrightarrow \forall x \in(\bigoplus G \leftarrow G s . G) . \exists !$ gs $\in$ listset $G$ s. $x=$ sum-list $g s$ $\langle$ proof $\rangle$

lemma AbGroup-inner-dirsum-pairwise-int0 :

$\llbracket \forall G \in$ set $G s$. AbGroup $G ;$ add-independent $S G s ; G \in$ set $G s ; G^{\prime} \in$ set $G s$; $G \neq G^{\prime} \rrbracket \Longrightarrow G \cap G^{\prime}=0$ $\langle$ proof $\rangle$

lemma AbGroup-inner-dirsum-pairwise-int0-double :

assumes AbGroup $G$ AbGroup $G^{\prime}$ add-independent $S\left[G, G^{\prime}\right]$

shows $G \cap G^{\prime}=0$

$\langle$ proof $\rangle$

\subsubsection{Element decomposition and projection}

definition inner-dirsum-el-decomp ::

'g::ab-group-add set list $\Rightarrow\left({ }^{\prime} g \Rightarrow{ }^{\prime} g\right.$ list $)(\bigoplus-\leftarrow)$

where $\bigoplus G s \leftarrow=(\lambda x$. if $x \in(\bigoplus G \leftarrow G s$. $G)$

then THE gs. gs $\in$ listset Gs $\wedge x=$ sum-list gs else []) 
abbreviation inner-dirsum-el-decomp-double ::

'g::ab-group-add set $\Rightarrow{ }^{\prime} g$ set $\Rightarrow\left({ }^{\prime} g \Rightarrow{ }^{\prime} g\right.$ list $)(-\oplus-\leftarrow)$ where $G \oplus H \leftarrow \equiv \bigoplus[G, H] \leftarrow$

abbreviation inner-dirsum-el-decomp-nth ::

'g::ab-group-add set list $\Rightarrow$ nat $\Rightarrow\left({ }^{\prime} g \Rightarrow{ }^{\prime} g\right)(\bigoplus-\downarrow-)$

where $\bigoplus G s \downarrow n \equiv$ restricto $(\lambda x .(\bigoplus G s \leftarrow x) ! n)(\bigoplus G \leftarrow G s . G)$

lemma AbGroup-inner-dirsum-el-decompI :

$\llbracket \forall G \in$ set Gs. AbGroup $G ;$ add-independent $S$ Gs; $x \in(\bigoplus G \leftarrow G s . G) \rrbracket$ $\langle$ proof $\rangle$

$\Longrightarrow(\bigoplus G s \leftarrow x) \in$ listset $G s \wedge x=$ sum-list $(\bigoplus G s \leftarrow x)$

lemma (in AbGroup) abSubgroup-inner-dirsum-el-decomp-set :

$\llbracket \forall H \in$ set Hs. Subgroup $H ;$ add-independent $S H s ; x \in(\bigoplus H \leftarrow H s . H) \rrbracket$

$\Longrightarrow \operatorname{set}(\bigoplus H s \leftarrow x) \subseteq G$

$\langle$ proof $\rangle$

lemma AbGroup-inner-dirsum-el-decomp-eq :

$\llbracket \forall G \in$ set Gs. AbGroup $G$; add-independent $S$ Gs; $x \in(\bigoplus G \leftarrow G s . G)$; $\langle$ proof $\rangle$

gs $\in$ listset $G s ; x=$ sum-list $g s \rrbracket \Longrightarrow(\bigoplus G s \leftarrow x)=g s$

lemma AbGroup-inner-dirsum-el-decomp-plus :

assumes $\forall G \in$ set Gs. AbGroup $G$ add-independent $S$ Gs $x \in(\bigoplus G \leftarrow G s . G)$ $y \in(\bigoplus G \leftarrow G s . G)$

shows $\quad(\bigoplus G s \leftarrow(x+y))=[a+b .(a, b) \leftarrow z i p(\bigoplus G s \leftarrow x)(\bigoplus G s \leftarrow y)]$ $\langle$ proof $\rangle$

lemma AbGroup-length-inner-dirsum-el-decomp :

$\llbracket \forall G \in$ set Gs. AbGroup $G ;$ add-independent $S$ Gs; $x \in(\bigoplus G \leftarrow G s . G) \rrbracket$ $\langle$ proof $\rangle$

$\Longrightarrow$ length $(\bigoplus G s \leftarrow x)=$ length $G s$

lemma AbGroup-inner-dirsum-el-decomp-in-nth :

assumes $\forall G \in$ set Gs. AbGroup $G$ add-independentS Gs $n<$ length $G$ s $x \in G s ! n$

shows $(\bigoplus G s \leftarrow x)=($ replicate $($ length Gs $) 0)[n:=x]$

$\langle$ proof $\rangle$

lemma AbGroup-inner-dirsum-el-decomp-nth-in-nth :

$\llbracket \forall G \in$ set Gs. AbGroup $G$; add-independentS $G s ; k<$ length $G s$; $\langle$ proof $\rangle$

$n<$ length $G s ; x \in G s ! n \rrbracket \Longrightarrow(\bigoplus G s \downarrow k) x=($ if $k=n$ then $x$ else 0$)$

lemma AbGroup-inner-dirsum-el-decomp-nth-id-on-nth :

$\llbracket \forall G \in$ set Gs. AbGroup $G$; add-independentS Gs; $n<$ length Gs; $x \in G s ! n \rrbracket$ $\langle$ proof $\rangle$

$\Longrightarrow(\bigoplus G s \downarrow n) x=x$ 
lemma AbGroup-inner-dirsum-el-decomp-nth-onto-nth :

assumes $\forall G \in$ set Gs. AbGroup $G$ add-independentS Gs $n<$ length $G$ s shows $\quad(\bigoplus G s \downarrow n) \cdot(\bigoplus G \leftarrow G s . G)=G s ! n$

$\langle$ proof $\rangle$

lemma AbGroup-inner-dirsum-subset-proj-eq-0 :

assumes $G s \neq[] \forall G \in$ set Gs. AbGroup $G$ add-independentS Gs

$X \subseteq(\bigoplus G \leftarrow G s . G) \forall i<$ length Gs. $(\bigoplus G s \downarrow i)$ ' $X=0$

shows $X=0$

$\langle$ proof $\rangle$

lemma GroupEnd-inner-dirsum-el-decomp-nth :

assumes $\forall G \in$ set Gs. AbGroup $G$ add-independentS Gs $n<$ length $G$ s

shows GroupEnd $(\bigoplus G \leftarrow G s . G)(\bigoplus G s \downarrow n)$

$\langle$ proof $\rangle$

\section{$2.6 \quad$ Rings}

\subsubsection{Preliminaries}

lemma (in ring-1) map-times-neg1-eq-map-uminus : $[(-1) * r . r \leftarrow r s]=[-r . r \leftarrow r s]$ $\langle$ proof $\rangle$

\subsubsection{Locale and basic facts}

Define a Ring1 to be a multiplicatively closed additive subgroup of UNIV for the ring-1 class.

locale Ring1 = Group $R$

for $R::{ }^{\prime} r::$ ring-1 set

+ assumes one-closed $: 1 \in R$

and mult-closed: $\bigwedge r s . r \in R \Longrightarrow s \in R \Longrightarrow r * s \in R$

begin

lemma AbGroup : AbGroup $R$

$\langle$ proof $\rangle$

lemmas zero-closed = zero-closed

lemmas add-closed $\quad=$ add-closed

lemmas neg-closed = neg-closed

lemmas diff-closed = diff-closed

lemmas zip-add-closed = zip-add-closed

lemmas sum-closed = AbGroup.sum-closed [OF AbGroup]

lemmas sum-list-closed = sum-list-closed

lemmas sum-list-closed-prod $=$ sum-list-closed-prod

lemmas list-diff-closed = list-diff-closed

abbreviation Subring1 :: 'r set $\Rightarrow$ bool where Subring1 $S \equiv \operatorname{Ring} 1 S \wedge S \subseteq R$

lemma Subring1D1: Subring1 $S \Longrightarrow$ Ring1 $S\langle$ proof $\rangle$ 
end

lemma (in ring-1) full-Ring1 : Ring1 UNIV

$\langle$ proof $\rangle$

\subsection{The group ring}

\subsubsection{Definition and basic facts}

Realize the group ring as the set of almost-every-zero functions from group to ring. One can recover the usual notion of group ring element by considering such a function to send group elements to their coefficients. Here the codomain of such functions is not restricted to some Ring1 subset since we will not be interested in having the ability to change the ring of scalars for a group ring.

context Group

begin

abbreviation group-ring :: ('a::zero, 'g) aezfun set where group-ring $\equiv$ aezfun-setspan $G$

lemmas group-ringD = aezfun-setspan-def $[$ of $G]$

lemma $R G$-one-closed : (1::('r::zero-neq-one, $\left.{ }^{\prime} g\right)$ aezfun $) \in$ group-ring $\langle$ proof $\rangle$

lemma $R G$-zero-closed : (0::('r::zero,'g) aezfun $) \in$ group-ring $\langle$ proof $\rangle$

lemma $R G$-n0 : group-ring $\neq\left(0::\left({ }^{\prime} r:: z e r o-n e q-o n e,{ }^{\prime} g\right)\right.$ aezfun set $)$ $\langle$ proof $\rangle$

lemma $R G$-mult-closed :

defines $R G: R G \equiv$ group-ring $::\left({ }^{\prime} r::\right.$ ring-1, 'g) aezfun set shows $x \in R G \Longrightarrow y \in R G \Longrightarrow x * y \in R G$

$\langle$ proof $\rangle$

lemma Ring1-RG :

defines $R G: R G \equiv$ group-ring $::\left({ }^{\prime} r::\right.$ ring-1, ' $g$ ) aezfun set shows Ring1 $R G$ $\langle$ proof $\rangle$

lemma $R G$-aezdeltafun-closed :

defines $R G: R G \equiv$ group-ring $::\left({ }^{\prime} r::\right.$ ring-1, ' $g$ ) aezfun set assumes $g \in G$

shows $\quad r \delta \delta g \in R G$

$\langle$ proof $\rangle$ 
lemma $R G$-aezdeltaOfun-closed : (r::'r::ring-1) $\delta \delta 0 \in$ group-ring

$\langle$ proof $\rangle$

lemma $R G$-sum-list-rddg-closed :

defines $R G: R G \equiv$ group-ring :: ('r::ring-1, 'g) aezfun set

assumes set (map snd rgs) $\subseteq$ G

shows $\left(\sum(r, g) \leftarrow r g s . r \delta \delta g\right) \in R G$

$\langle$ proof $\rangle$

lemmas RG-el-decomp-aezdeltafun = aezfun-setspan-el-decomp-aezdeltafun $[$ of - $G]$

lemma Subgroup-imp-Subring :

fixes $H:{ }^{\prime} g$ set

and $F H::\left({ }^{\prime} r::\right.$ ring-1, $\left.{ }^{\prime} g\right)$ aezfun set

and $F G::\left({ }^{\prime} r,{ }^{\prime} g\right)$ aezfun set

defines $F H \equiv$ Group.group-ring $H$

and $F G \equiv$ group-ring

shows Subgroup $H \Longrightarrow$ Ring1.Subring1 FG FH

$\langle$ proof $\rangle$

end

lemma (in FinGroup) group-ring-spanning-set :

$\exists$ gs. distinct $g s \wedge$ set $g s=G$

$\wedge(\forall f \in$ (group-ring :: ('b::semiring-1, 'g) aezfun set). $\exists b s$ $\langle$ proof $\rangle$

length bs = length gs $\wedge f=\left(\sum(b, g) \leftarrow z i p\right.$ bs gs. $\left.\left.\left(\begin{array}{llll}b & \delta \delta & 0\end{array}\right) *\left(\begin{array}{lll}1 & \delta \delta & g\end{array}\right)\right)\right)$

\subsubsection{Projecting almost-everywhere-zero functions onto a group ring}

context Group

begin

abbreviation $R G$-proj $\equiv$ aezfun-setspan-proj $G$

lemmas $R G$-proj-in- $R G \quad=$ aezfun-setspan-proj-in-setspan

lemmas $R G$-proj-sum-list-prod = aezfun-setspan-proj-sum-list-prod $[$ of $G]$

lemma $R G$-proj-mult-leftdelta' :

fixes $\quad r s::{ }^{\prime} r::\{$ comm-monoid-add,mult-zero $\}$

shows $g \in G \Longrightarrow R G$-proj $\left(r \delta \delta g *\left(s \delta \delta g^{\prime}\right)\right)=r \delta \delta g * R G$-proj $\left(s \delta \delta g^{\prime}\right)$

$\langle$ proof $\rangle$

lemma $R G$-proj-mult-leftdelta :

fixes $r::{ }^{\prime} r::$ semiring-1

assumes $g \in G$

shows $R G$-proj $((r \delta \delta g) * x)=r \delta \delta g * R G$-proj $x$ 
$\langle$ proof $\rangle$

lemma $R G$-proj-mult-rightdelta' ${ }^{\prime}$ :

fixes $\quad r s::{ }^{\prime} r::\{c o m m-m o n o i d-a d d$, mult-zero $\}$

assumes $g^{\prime} \in G$

shows $R G$-proj $\left(r \delta \delta g *\left(s \delta \delta g^{\prime}\right)\right)=R G$-proj $(r \delta \delta g) *\left(s \delta \delta g^{\prime}\right)$

$\langle$ proof $\rangle$

lemma $R G$-proj-mult-rightdelta :

fixes $r::{ }^{\prime} r::$ semiring-1

assumes $g \in G$

shows $R G$-proj $(x *(r \delta \delta g))=(R G$-proj $x) *(r \delta \delta g)$

$\langle$ proof $\rangle$

lemma $R G$-proj-mult-right :

$x \in\left(\right.$ group-ring $::\left({ }^{\prime} r::\right.$ ring-1, 'g) aezfun set)

$\langle$ proof $\rangle$

$\Longrightarrow R G-\operatorname{proj}(y * x)=R G-\operatorname{proj} y * x$

end

\section{Modules}

\subsection{Locales and basic facts}

\subsubsection{Locales}

locale scalar-mult $=$

fixes smult :: 'r::ring-1 $\Rightarrow$ 'm::ab-group-add $\Rightarrow{ }^{\prime} m$ (infixr • 70)

locale $R$-scalar-mult $=$ scalar-mult smult + Ring1 $R$

for $R \quad::{ }^{\prime} r::$ ring-1 set

and smult :: $r \Rightarrow{ }^{\prime} m:: a b$-group-add $\Rightarrow{ }^{\prime} m$ (infixr $\left.\cdot 70\right)$

lemma (in scalar-mult) R-scalar-mult : R-scalar-mult UNIV

$\langle$ proof $\rangle$

lemma (in R-scalar-mult) Ring1 : Ring1 $R\langle$ proof $\rangle$

locale $R$ Module $=R$-scalars?: R-scalar-mult $R$ smult + VecGroup?: Group $M$

for $R \quad::{ }^{\prime} r::$ ring-1 set

and smult $:{ }^{\prime} r \Rightarrow{ }^{\prime} m:: a b$-group-add $\Rightarrow{ }^{\prime} m$ (infixr $\cdot 70$ )

and $M \quad::{ }^{\prime} m$ set

+ assumes smult-closed $: \llbracket r \in R ; m \in M \rrbracket \Longrightarrow r \cdot m \in M$

and smult-distrib-left $[$ simp $]: \llbracket r \in R ; m \in M ; n \in M \rrbracket$

$\Longrightarrow r \cdot(m+n)=r \cdot m+r \cdot n$

and smult-distrib-right $[$ simp $]: \llbracket r \in R ; s \in R ; m \in M \rrbracket$

$\Longrightarrow(r+s) \cdot m=r \cdot m+s \cdot m$

and smult-assoc $[\operatorname{simp}]: \llbracket r \in R ; s \in R ; m \in M \rrbracket$ 
$\begin{aligned} & \Longrightarrow r \cdot s \cdot m=(r * s) \cdot m \\ & \text { and one-smult }[\text { simp }]: m \in M \Longrightarrow 1 \cdot m=m\end{aligned}$

lemmas RModuleI $=$ RModule.intro[OF R-scalar-mult.intro $]$

locale Module $=$ RModule UNIV smult $M$

for smult :: 'r::ring-1 $\Rightarrow$ 'm::ab-group-add $\Rightarrow{ }^{\prime} m$ (infixr · 70)

and $M \quad::{ }^{\prime} m$ set

lemmas ModuleI = RModuleI $[$ of UNIV, OF full-Ring1, THEN Module.intro $]$

\subsubsection{Basic facts}

lemma trivial-RModule :

fixes smult :: 'r::ring-1 $\Rightarrow$ 'm::ab-group-add $\Rightarrow{ }^{\prime} m$ (infixr $\left.\cdot 70\right)$

assumes Ring1 $R \forall r \in R$. smult $r\left(0::^{\prime} m:: a b\right.$-group-add $)=0$

shows RModule $R$ smult $\left(0::^{\prime} m\right.$ set $)$

$\langle$ proof $\rangle$

context RModule

begin

abbreviation RSubmodule :: 'm set $\Rightarrow$ bool

where RSubmodule $N \equiv$ RModule $R$ smult $N \wedge N \subseteq M$

lemma Group : Group $M$

$\langle$ proof $\rangle$

lemma Subgroup-RSubmodule : RSubmodule $N \Longrightarrow$ Subgroup $N$

$\langle$ proof $\rangle$

lemma AbGroup : AbGroup $M$

$\langle$ proof $\rangle$

lemmas zero-closed = zero-closed

lemmas diff-closed $=$ diff-closed

lemmas set-plus-closed $=$ set-plus-closed

lemmas sum-closed = AbGroup.sum-closed $[$ OF AbGroup $]$

lemma map-smult-closed :

$r \in R \Longrightarrow$ set $m s \subseteq M \Longrightarrow \operatorname{set}(\operatorname{map}((\cdot) r) m s) \subseteq M$

$\langle$ proof $\rangle$

lemma zero-smult : $m \in M \Longrightarrow 0 \cdot m=0$

$\langle$ proof $\rangle$

lemma smult-zero : $r \in R \Longrightarrow r \cdot 0=0$

$\langle$ proof $\rangle$ 
lemma neg-smult $: r \in R \Longrightarrow m \in M \Longrightarrow(-r) \cdot m=-(r \cdot m)$ $\langle$ proof $\rangle$

lemma neg-eq-neg1-smult : $m \in M \Longrightarrow(-1) \cdot m=-m$ $\langle$ proof $\rangle$

lemma smult-neg $: r \in R \Longrightarrow m \in M \Longrightarrow r \cdot(-m)=-(r \cdot m)$ $\langle$ proof $\rangle$

lemma smult-distrib-left-diff :

$\llbracket r \in R ; m \in M ; n \in M \rrbracket \Longrightarrow r \cdot(m-n)=r \cdot m-r \cdot n$ $\langle$ proof $\rangle$

lemma smult-distrib-right-diff :

$\llbracket r \in R ; s \in R ; m \in M \rrbracket \Longrightarrow(r-s) \cdot m=r \cdot m-s \cdot m$ $\langle$ proof $\rangle$

lemma smult-sum-distrib :

assumes $r \in R$

shows finite $A \Longrightarrow f^{\prime} A \subseteq M \Longrightarrow r \cdot\left(\sum a \in A . f a\right)=\left(\sum a \in A \cdot r \cdot f a\right)$ $\langle$ proof $\rangle$

lemma sum-smult-distrib :

assumes $m \in M$

shows finite $A \Longrightarrow f^{\prime} A \subseteq R \Longrightarrow\left(\sum a \in A . f a\right) \cdot m=\left(\sum a \in A .(f a) \cdot m\right)$ $\langle$ proof $\rangle$

lemma smult-sum-list-distrib :

$r \in R \Longrightarrow$ set $m s \subseteq M \Longrightarrow r \cdot($ sum-list $m s)=\left(\sum m \leftarrow m s . r \cdot m\right)$ $\langle$ proof $\rangle$

lemma sum-list-prod-map-smult-distrib :

$m \in M \Longrightarrow \operatorname{set}(\operatorname{map}$ (case-prod $f)$ xys $) \subseteq R$ $\langle$ proof $\rangle$

$\Longrightarrow\left(\sum(x, y) \leftarrow x y s . f x y\right) \cdot m=\left(\sum(x, y) \leftarrow x y s . f x y \cdot m\right)$

lemma RSubmoduleI :

assumes Subgroup $N \wedge r n . r \in R \Longrightarrow n \in N \Longrightarrow r \cdot n \in N$

shows RSubmodule $N$

$\langle$ proof $\rangle$

end

lemma (in $R$-scalar-mult) listset-RModule-Rsmult-closed :

$\llbracket \forall M \in$ set $M s$. RModule $R$ smult $M ; r \in R ; m s \in$ listset $M s \rrbracket$ $\Longrightarrow[r \cdot m . m \leftarrow m s] \in$ listset $M s$

$\langle$ proof $\rangle$

context Module 
begin

abbreviation Submodule :: 'm set $\Rightarrow$ bool

where Submodule $\equiv$ RModule.RSubmodule UNIV smult $M$

lemmas AbGroup = AbGroup

lemmas SubmoduleI $=$ RSubmoduleI

end

\subsubsection{Module and submodule instances}

lemma (in $R$-scalar-mult) trivial-RModule :

$(\bigwedge r . r \in R \Longrightarrow r \cdot 0=0) \Longrightarrow$ RModule $R$ smult 0

$\langle$ proof $\rangle$

context RModule

begin

lemma trivial-RSubmodule : RSubmodule 0 $\langle$ proof $\rangle$

lemma RSubmodule-set-plus :

assumes RSubmodule L RSubmodule $N$

shows RSubmodule $(L+N)$

$\langle$ proof $\rangle$

lemma RSubmodule-sum-list :

$(\forall N \in$ set Ns. RSubmodule $N) \Longrightarrow$ RSubmodule $\left(\sum N \leftarrow N s . N\right)$

$\langle$ proof $\rangle$

lemma RSubmodule-inner-dirsum :

assumes $(\forall N \in$ set Ns. RSubmodule $N)$

shows $\quad$ RSubmodule $(\bigoplus N \leftarrow N s . N)$

$\langle$ proof $\rangle$

lemma RModule-inner-dirsum :

$(\forall N \in$ set Ns. RSubmodule $N) \Longrightarrow$ RModule $R$ smult $(\bigoplus N \leftarrow N s . N)$ $\langle$ proof $\rangle$

lemma SModule-restrict-scalars :

assumes Subring1 $S$

shows RModule $S$ smult $M$

$\langle$ proof $\rangle$

end 


\subsection{Linear algebra in modules}

\subsubsection{Linear combinations: lincomb}

context scalar-mult

begin

definition lincomb :: 'r list $\Rightarrow{ }^{\prime} m$ list $\Rightarrow{ }^{\prime} m$ (infix .. 70)

where $r s \cdot m s=\left(\sum(r, m) \leftarrow z i p\right.$ rs ms. $\left.r \cdot m\right)$

Note: zip will truncate if lengths of coefficient and vector lists differ.

lemma lincomb-Nil : rs $=[] \vee m s=[] \Longrightarrow r s \cdot m s=0$

$\langle$ proof $\rangle$

lemma lincomb-singles : $[a] \cdot[m]=a \cdot m$

$\langle$ proof $\rangle$

lemma lincomb-Cons : $(r \#$ \#s $) \cdot(m \# m s)=r \cdot m+r s \cdot m s$ $\langle$ proof $\rangle$

lemma lincomb-append :

length $r s=$ length $m s \Longrightarrow(r s @ s s) \cdot(m s @ n s)=r s \cdot m s+s s \cdot n s$ $\langle$ proof $\rangle$

lemma lincomb-append-left :

$(r s @ s s) \cdot m s=r s \cdot m s+s s \cdot \cdot \operatorname{drop}($ length $r s) m s$

$\langle$ proof $\rangle$

lemma lincomb-append-right :

$r s \cdot .(m s @ n s)=r s \cdot . m s+($ drop $($ length $m s) r s) \cdot \cdot n s$

$\langle$ proof $\rangle$

lemma lincomb-conv-take-right : rs $\cdot$ ms $=r s \cdot$ take (length rs) $\mathrm{ms}$ $\langle$ proof $\rangle$

end

context RModule

begin

lemmas lincomb-Nil = lincomb-Nil

lemmas lincomb-Cons $=$ lincomb-Cons

lemma lincomb-closed : set $r s \subseteq R \Longrightarrow$ set $m s \subseteq M \Longrightarrow r s \cdot m s \in M$ $\langle$ proof $\rangle$

lemma smult-lincomb :

$\llbracket$ set $r s \subseteq R ; s \in R ;$ set $m s \subseteq M \rrbracket \Longrightarrow s \cdot(r s \cdot \cdot m s)=[s * r . r \leftarrow r s] \cdot \cdot m s$ $\langle$ proof $\rangle$ 
lemma neg-lincomb :

set $r s \subseteq R \Longrightarrow$ set $m s \subseteq M \Longrightarrow-(r s \cdot m s)=[-r . r \leftarrow r s] \cdots m s$

$\langle$ proof $\rangle$

lemma lincomb-sum-left :

【set $r s \subseteq R$; set $s s \subseteq R$; set $m s \subseteq M$; length $r s \leq$ length ss 》 $\langle$ proof $\rangle$

$\Longrightarrow[r+s .(r, s) \leftarrow z i p r s s s] \cdot m s=r s \cdot m s+($ take $($ length $r s) s s) \cdot m s$

lemma lincomb-sum :

assumes set $r s \subseteq R$ set $s s \subseteq R$ set $m s \subseteq M$ length $r s \leq$ length ss

shows $r s \cdot m s+s s \cdot m s$

$\langle$ proof $\rangle$

$$
=([a+b .(a, b) \leftarrow z i p r s s s] @(d r o p(\text { length } r s) s s)) \cdots m s
$$

lemma lincomb-diff-left :

【set $r s \subseteq R ;$ set $s s \subseteq R ;$ set $m s \subseteq M$; length $r s \leq$ length $s s \rrbracket$ $\langle$ proof $\rangle$

$\Longrightarrow[r-s .(r, s) \leftarrow z i p r s s s] \cdot m s=r s \cdot m s-($ take $($ length $r s) s s) \cdot m s$

lemma lincomb-replicate-left :

$r \in R \Longrightarrow$ set $m s \subseteq M \Longrightarrow($ replicate $k r) \cdot \cdot m s=r \cdot\left(\sum m \leftarrow(\right.$ take $\left.k m s) . m\right)$ $\langle$ proof $\rangle$

lemma lincomb-replicate0-left : set $m s \subseteq M \Longrightarrow($ replicate $k 0) \cdot \cdot m s=0$

$\langle$ proof $\rangle$

lemma lincomb-0coeffs : set $m s \subseteq M \Longrightarrow \forall s \in$ set rs. $s=0 \Longrightarrow$ rs $\cdot m s=0$ $\langle$ proof $\rangle$

lemma delta-scalars-lincomb-eq-nth :

set $m s \subseteq M \Longrightarrow n<$ length $m s$

$\langle$ proof $\rangle$

$$
\Longrightarrow((\text { replicate }(\text { length } m s) 0)[n:=1]) \cdots m s=m s ! n
$$

lemma lincomb-obtain-same-length-Rcoeffs :

set $r s \subseteq R \Longrightarrow$ set $m s \subseteq M$

$\Longrightarrow \exists$ ss. set $s s \subseteq R \wedge$ length ss $=$ length $\mathrm{ms}$

$\wedge$ take (length rs) ss = take (length $m s) r s \wedge r s \cdot m s=s s \cdot m s$

$\langle$ proof $\rangle$

lemma lincomb-concat :

list-all2 ( $\lambda$ rs ms. length $r s=$ length $\mathrm{ms})$ rss mss $\langle$ proof $\rangle$

$\Longrightarrow($ concat rss $) \cdot($ concat $m s s)=\left(\sum(\right.$ rs, ms $) \leftarrow$ zip rss mss. rs $\left.\cdots m s\right)$

lemma lincomb-snoc0 : set $m s \subseteq M \Longrightarrow(a s @[0]) \cdot \cdots m s=a s \cdot m s$ $\langle$ proof $\rangle$ 
lemma lincomb-strip-while-Ocoeffs :

assumes set $m s \subseteq M$

shows (strip-while $((=) 0)$ as $) \cdot m s=a s \cdot m s$

$\langle$ proof $\rangle$

end

lemmas (in Module) lincomb-obtain-same-length-coeffs = lincomb-obtain-same-length-Rcoeffs

lemmas (in Module) lincomb-concat $\quad=$ lincomb-concat

\subsubsection{Spanning: RSpan and Span}

context $R$-scalar-mult

begin

primrec $R$ Span :: 'm list $\Rightarrow{ }^{\prime} m$ set

where $R$ Span [] =0

$\mid R \operatorname{Span}(m \# m s)=\{r \cdot m \mid r . r \in R\}+R$ Span $m s$

lemma RSpan-single : RSpan $[m]=\{r \cdot m \mid r . r \in R\}$

$\langle$ proof $\rangle$

lemma RSpan-Cons : RSpan $(m \# m s)=R \operatorname{Span}[m]+R \operatorname{Span} m s$

$\langle$ proof $\rangle$

lemma in-RSpan-obtain-same-length-coeffs :

$n \in R$ Span $m s \Longrightarrow \exists$ rs. set $r s \subseteq R \wedge$ length $r s=$ length $m s \wedge n=r s \cdot m s$ $\langle$ proof $\rangle$

lemma in-RSpan-Cons-obtain-same-length-coeffs :

$n \in R$ Span $(m \# m s) \Longrightarrow \exists r$ rs. set $(r \# r s) \subseteq R \wedge$ length $r s=$ length $m s$

$\wedge n=r \cdot m+r s \cdot m s$

$\langle$ proof $\rangle$

lemma RSpanD-lincomb :

$R$ Span $m s=\{r s \cdot m s \mid$ rs. set $r s \subseteq R \wedge$ length $r s=$ length $m s\}$ $\langle$ proof $\rangle$

lemma RSpan-append : RSpan $(m s @ n s)=R \operatorname{Span} m s+R \operatorname{Span} n s$ $\langle$ proof $\rangle$

end

context scalar-mult

begin

abbreviation Span $\equiv R$-scalar-mult.RSpan UNIV smult

lemmas Span-append $=R$-scalar-mult.RSpan-append $[O F$ R-scalar-mult, of smult $]$ 
lemmas SpanD-lincomb

$=R$-scalar-mult.RSpanD-lincomb [OF R-scalar-mult, of smult $]$

lemmas in-Span-obtain-same-length-coeffs

$=R$-scalar-mult.in-RSpan-obtain-same-length-coeffs [

OF R-scalar-mult, of - smult

]

end

context RModule

begin

lemma RSpan-contains-spanset-single : $m \in M \Longrightarrow m \in R \operatorname{Span}[m]$

$\langle$ proof $\rangle$

lemma RSpan-single-nonzero $: m \in M \Longrightarrow m \neq 0 \Longrightarrow R \operatorname{Span}[m] \neq 0$ $\langle$ proof $\rangle$

lemma Group-RSpan-single :

assumes $m \in M$

shows Group (RSpan $[m])$

$\langle$ proof $\rangle$

lemma Group-RSpan : set $m s \subseteq M \Longrightarrow \operatorname{Group}(R \operatorname{Span} m s)$

$\langle$ proof $\rangle$

lemma RSpanD-lincomb-arb-len-coeffs :

set $m s \subseteq M \Longrightarrow R$ Span $m s=\{r s \cdots m s \mid r s$. set $r s \subseteq R\}$

$\langle$ proof $\rangle$

lemma RSpanI-lincomb-arb-len-coeffs :

set $r s \subseteq R \Longrightarrow$ set $m s \subseteq M \Longrightarrow r s \cdot m s \in R$ Span $m s$

$\langle$ proof $\rangle$

lemma RSpan-contains-RSpans-Cons-left :

set $m s \subseteq M \Longrightarrow R \operatorname{Span}[m] \subseteq R \operatorname{Span}(m \# m s)$

$\langle$ proof $\rangle$

lemma RSpan-contains-RSpans-Cons-right :

$m \in M \Longrightarrow R$ Span $m s \subseteq R \operatorname{Span}(m \# m s)$

$\langle$ proof $\rangle$

lemma RSpan-contains-RSpans-append-left :

set $n s \subseteq M \Longrightarrow R$ Span ms $\subseteq$ Rpan $(m s @ n s)$

$\langle$ proof $\rangle$

lemma RSpan-contains-spanset : set $m s \subseteq M \Longrightarrow$ set $m s \subseteq R$ Span ms $\langle$ proof $\rangle$ 
lemma RSpan-contains-spanset-append-left :

set $m s \subseteq M \Longrightarrow$ set $n s \subseteq M \Longrightarrow$ set $m s \subseteq R \operatorname{Span}(m s @ n s)$ $\langle$ proof $\rangle$

lemma $R$ Span-contains-spanset-append-right :

set $m s \subseteq M \Longrightarrow$ set $n s \subseteq M \Longrightarrow$ set $n s \subseteq R \operatorname{Span}(m s @ n s)$

$\langle$ proof $\rangle$

lemma RSpan-zero-closed : set $m s \subseteq M \Longrightarrow 0 \in R$ Span ms $\langle$ proof $\rangle$

lemma RSpan-single-closed $: m \in M \Longrightarrow R \operatorname{Span}[m] \subseteq M$

$\langle$ proof $\rangle$

lemma RSpan-closed : set $m s \subseteq M \Longrightarrow R$ Span $m s \subseteq M$

$\langle$ proof $\rangle$

lemma RSpan-smult-closed :

assumes $r \in R$ set $m s \subseteq M n \in R$ Span ms

shows $r \cdot n \in R$ Span $m s$

$\langle$ proof $\rangle$

lemma RSpan-add-closed :

assumes set $m s \subseteq M n \in R$ Span ms $n^{\prime} \in R$ Span $m s$

shows $n+n^{\prime} \in R$ Span ms

$\langle$ proof $\rangle$

lemma RSpan-lincomb-closed :

$\llbracket$ set $r s \subseteq R$; set $m s \subseteq M$; set $n s \subseteq R$ Span $m s \rrbracket \Longrightarrow r s \cdot n s \in R$ Span $m s$ $\langle$ proof $\rangle$

lemma $R$ SpanI : set $m s \subseteq M \Longrightarrow M \subseteq R$ Span $m s \Longrightarrow M=R$ Span ms $\langle$ proof $\rangle$

lemma RSpan-contains-RSpan-take :

set $m s \subseteq M \Longrightarrow R \operatorname{Span}($ take $k m s) \subseteq R$ Span $m s$

$\langle$ proof $\rangle$

lemma RSubmodule-RSpan-single :

assumes $m \in M$

shows RSubmodule (RSpan $[m])$

$\langle$ proof $\rangle$

lemma RSubmodule-RSpan : set $m s \subseteq M \Longrightarrow$ RSubmodule (RSpan ms) $\langle$ proof $\rangle$

lemma RSpan-RSpan-closed :

set $m s \subseteq M \Longrightarrow$ set $n s \subseteq R$ Span $m s \Longrightarrow$ RSpan $n s \subseteq R$ Span $m s$ 


$$
\langle\text { proof }\rangle
$$

lemma spanset-reduce-Cons :

set $m s \subseteq M \Longrightarrow m \in R$ Span $m s \Longrightarrow R \operatorname{Span}(m \# m s)=R$ Span $m s$

$\langle$ proof $\rangle$

lemma RSpan-replace-hd :

assumes $n \in M$ set $m s \subseteq M m \in R \operatorname{Span}(n \# m s)$

shows $R \operatorname{Span}(m \# m s) \subseteq R \operatorname{Span}(n \# m s)$

$\langle$ proof $\rangle$

end

lemmas (in scalar-mult)

Span-Cons $=R$-scalar-mult.RSpan-Cons $[O F R$-scalar-mult, of smult $]$

context Module

begin

lemmas SpanD-lincomb-arb-len-coeffs $=$ RSpanD-lincomb-arb-len-coeffs

lemmas SpanI $=R$ SpanI

lemmas SpanI-lincomb-arb-len-coeffs = RSpanI-lincomb-arb-len-coeffs

lemmas Span-contains-Spans-Cons-right = RSpan-contains-RSpans-Cons-right

lemmas Span-contains-spanset = RSpan-contains-spanset

lemmas Span-contains-spanset-append-left $=$ RSpan-contains-spanset-append-left

lemmas Span-contains-spanset-append-right $=R$ Span-contains-spanset-append-right

lemmas Span-closed

= RSpan-closed

lemmas Span-smult-closed

$=$ RSpan-smult-closed

lemmas Span-contains-Span-take

= RSpan-contains-RSpan-take

lemmas Span-replace-hd

$=R$ Span-replace- $h d$

lemmas Submodule-Span

$=$ RSubmodule-RSpan

end

\subsubsection{Finitely generated modules}

context $R$-scalar-mult

begin

abbreviation $R$-fingen $M \equiv(\exists m s$. set $m s \subseteq M \wedge R$ Span $m s=M)$

Similar to definition of card for finite sets, we default $\operatorname{dim}$ to 0 if no finite spanning set exists. Note that $R$ Span []$=0$ implies that $\operatorname{dim}-R\left\{0::^{\prime} b\right\}=$ $\left(0::^{\prime} a\right)$.

definition $\operatorname{dim}-R::{ }^{\prime} m$ set $\Rightarrow$ nat

where dim- $R M=$ (if $R$-fingen $M$ then (

LEAST $n . \exists m s$. length $m s=n \wedge$ set $m s \subseteq M \wedge R$ Span $m s=M$

) else 0) 
lemma dim-R-nonzero :

assumes $\operatorname{dim}-R M>0$

shows $M \neq 0$

$\langle$ proof $\rangle$

end

hide-const real-vector.dim

hide-const (open) Real-Vector-Spaces.dim

abbreviation (in scalar-mult) fingen $\equiv R$-scalar-mult.R-fingen UNIV smult abbreviation (in scalar-mult) dim $\equiv R$-scalar-mult.dim- $R$ UNIV smult

lemmas (in Module) dim-nonzero $=$ dim-R-nonzero

\subsection{4 $R$-linear independence}

context $R$-scalar-mult

begin

primrec $R$-lin-independent :: ' $m$ list $\Rightarrow$ bool where

$R$-lin-independent-Nil: R-lin-independent [] = True

$R$-lin-independent-Cons:

$R$-lin-independent $(m \# m s)=(R$-lin-independent $m s$

$\wedge(\forall r r s .(\operatorname{set}(r \# r s) \subseteq R \wedge(r \# r s) \cdot \cdot(m \# m s)=0) \longrightarrow r=0))$

lemma $R$-lin-independent-ConsI :

assumes $R$-lin-independent $m s$

$\bigwedge r$ rs. set $(r \# r s) \subseteq R \Longrightarrow(r \# r s) \cdot \cdot(m \# m s)=0 \Longrightarrow r=0$

shows R-lin-independent $(m \# m s)$

$\langle$ proof $\rangle$

lemma $R$-lin-independent-ConsD1 :

$R$-lin-independent $(m \# m s) \Longrightarrow R$-lin-independent $m s$

$\langle$ proof $\rangle$

lemma $R$-lin-independent-ConsD2 :

$\llbracket R$-lin-independent $(m \# m s) ;$ set $(r \# r s) \subseteq R ;(r \# r s) \cdot(m \# m s)=0 \rrbracket$

$\Longrightarrow r=0$

$\langle$ proof $\rangle$

end

context RModule

begin

lemma $R$-lin-independent-imp-same-scalars :

$\llbracket$ length $r s=$ length $s s ;$ length $r s \leq$ length $m s ;$ set $r s \subseteq R$; set $s s \subseteq R$; 

$\langle$ proof $\rangle$

set $m s \subseteq M ; R$-lin-independent $m s ; r s \cdot m s=s s \cdot m s \rrbracket \Longrightarrow r s=s s$

lemma $R$-lin-independent-obtain-unique-scalars :

$\llbracket$ set $m s \subseteq M ; R$-lin-independent $m s ; n \in R$ Span $m s \rrbracket$ $\langle$ proof $\rangle$

$\Longrightarrow(\exists$ ! rs. set $r s \subseteq R \wedge$ length $r s=$ length $m s \wedge n=r s \cdot m s)$

lemma $R$-lin-independentI-all-scalars :

set $m s \subseteq M \Longrightarrow$

$(\forall$ rs. set $r s \subseteq R \wedge$ length $r s=$ length $m s \wedge r s \cdot m s=0 \longrightarrow$ set $r s \subseteq 0)$

$\langle$ proof $\rangle$

$\Longrightarrow R$-lin-independent $m s$

lemma $R$-lin-independentI-concat-all-scalars :

defines eq-len: eq-len $\equiv \lambda x s$ ys. length $x s=$ length ys

assumes set (concat mss) $\subseteq M$

$\bigwedge$ rss. set (concat rss) $\subseteq R \Longrightarrow$ list-all2 eq-len rss mss

$\Longrightarrow($ concat $r s s) \cdot($ concat $m s s)=0 \Longrightarrow(\forall r s \in$ set rss. set $r s \subseteq 0)$

shows R-lin-independent (concat mss)

$\langle$ proof $\rangle$

lemma $R$-lin-independentD-all-scalars :

$\llbracket$ set $r s \subseteq R$; set $m s \subseteq M$; length $r s \leq$ length $m s ; R$-lin-independent ms;

$\langle$ proof $\rangle$

$r s \cdot m s=0 \rrbracket$ set $r s \subseteq 0$

lemma $R$-lin-independentD-all-scalars-nth :

assumes set $r s \subseteq R$ set $m s \subseteq M R$-lin-independent $m s$ rs $\cdot m s=0$

$k<\min$ (length rs) (length $\mathrm{ms}$ )

shows $r s ! k=0$

$\langle$ proof $\rangle$

lemma $R$-lin-dependent-dependence-relation :

set $m s \subseteq M \Longrightarrow \neg R$-lin-independent $m s$ $\langle$ proof $\rangle$

$\Longrightarrow \exists r s$. set $r s \subseteq R \wedge$ set $r s \neq 0 \wedge$ length $r s=$ length $m s \wedge r s \cdot m s=0$

lemma $R$-lin-independent-imp-distinct :

set $m s \subseteq M \Longrightarrow R$-lin-independent $m s \Longrightarrow$ distinct $m s$

$\langle$ proof $\rangle$

lemma $R$-lin-independent-imp-independent-take :

set $m s \subseteq M \Longrightarrow R$-lin-independent $m s \Longrightarrow R$-lin-independent (take $n$ ms)

$\langle$ proof $\rangle$

lemma $R$-lin-independent-Cons-imp-independent-RSpans :

assumes $m \in M R$-lin-independent ( $m \# m s)$

shows add-independentS [RSpan $[m], R$ Span $m s]$ 
$\langle$ proof $\rangle$

lemma hd0-imp-R-lin-dependent $: \neg R$-lin-independent $(0 \# m s)$

$\langle$ proof $\rangle$

lemma $R$-lin-independent-imp-hd-n0 : R-lin-independent $(m \# m s) \Longrightarrow m \neq 0$ $\langle$ proof $\rangle$

lemma $R$-lin-independent-imp-hd-independent-from-RSpan :

assumes $m \in M$ set $m s \subseteq M R$-lin-independent ( $m \# m s)$

shows $m \notin R$ Span $m s$

$\langle$ proof $\rangle$

lemma $R$-lin-independent-reduce :

assumes $n \in M$

shows $\quad$ set $m s \subseteq M \Longrightarrow R$-lin-independent ( $m s @ n \# n s$ )

$\langle$ proof $\rangle$

$\Longrightarrow R$-lin-independent ( $m s$ @ ns)

lemma $R$-lin-independent-vs-lincomb0 :

assumes set $(m s @ n \# n s) \subseteq M R$-lin-independent (ms @n\#ns)

set $(r s @ s \# s s) \subseteq R$ length $r s=$ length $m s$

$(r s @ s \# s s) \cdot \cdot(m s @ n \# n s)=0$

shows $s=0$

$\langle$ proof $\rangle$

lemma $R$-lin-independent-append-imp-independent-RSpans :

set $m s \subseteq M \Longrightarrow R$-lin-independent (ms@ns)

$\Longrightarrow$ add-independentS $[$ RSpan ms, RSpan ns]

$\langle$ proof $\rangle$

end

\subsubsection{Linear independence over $U N I V$}

context scalar-mult

begin

abbreviation lin-independent $m s$

$\equiv R$-scalar-mult.R-lin-independent UNIV smult ms

lemmas lin-independent-ConsI

$=R$-scalar-mult.R-lin-independent-ConsI $[$ OF R-scalar-mult, of smult $]$

lemmas lin-independent-ConsD1

$=R$-scalar-mult.R-lin-independent-ConsD1[OF R-scalar-mult, of smult $]$

end

context Module 


\section{begin}

lemmas lin-independent-imp-independent-take $=R$-lin-independent-imp-independent-take lemmas lin-independent-reduce $\quad=R$-lin-independent-reduce lemmas lin-independent-vs-lincomb0 $\quad=R$-lin-independent-vs-lincombo lemmas lin-dependent-dependence-relation $=R$-lin-dependent-dependence-relation lemmas lin-independent-imp-distinct $\quad=R$-lin-independent-imp-distinct

lemmas lin-independent-imp-hd-independent-from-Span $=R$-lin-independent-imp-hd-independent-from-RSpan

lemmas lin-independent-append-imp-independent-Spans

$$
=R \text {-lin-independent-append-imp-independent-RSpans }
$$

end

\subsubsection{Rank}

context $R$-scalar-mult

begin

definition $R$-finrank :: 'm set $\Rightarrow$ bool

where $R$-finrank $M=(\exists n . \forall$ ms. set $m s \subseteq M$

$\wedge R$-lin-independent $m s \longrightarrow$ length $m s \leq n)$

lemma $R$-finrankI :

$(\bigwedge m s$. set $m s \subseteq M \Longrightarrow R$-lin-independent $m s \Longrightarrow$ length $m s \leq n)$

$\langle$ proof $\rangle$

$\Longrightarrow R$-finrank $M$

lemma $R$-finrankD :

$R$-finrank $M \Longrightarrow \exists n . \forall m s$. set $m s \subseteq M \wedge R$-lin-independent $m s$

$\langle$ proof $\rangle$

$\longrightarrow$ length $m s \leq n$

lemma submodule-R-finrank : R-finrank $M \Longrightarrow N \subseteq M \Longrightarrow R$-finrank $N$ $\langle$ proof $\rangle$

end

context scalar-mult

begin

abbreviation finrank :: 'm set $\Rightarrow$ bool

where finrank $\equiv R$-scalar-mult.R-finrank UNIV smult

lemmas finrankI $=R$-scalar-mult.R-finrankI $[O F R$-scalar-mult, of - smult $]$

lemmas finrankD $=R$-scalar-mult.R-finrankD $[O F R$-scalar-mult, of smult $]$

lemmas submodule-finrank

$=R$-scalar-mult.submodule-R-finrank [OF R-scalar-mult, of smult $]$ 
end

\subsection{Module homomorphisms}

\subsubsection{Locales}

locale RModuleHom = Domain?: RModule $R$ smult $M$

+ Codomain?: scalar-mult smult ${ }^{\prime}$

+ GroupHom?: GroupHom MT

for $R \quad:{ }^{\prime} r::$ ring-1 set

and smult $::{ }^{\prime} r \Rightarrow{ }^{\prime} m:: a b$-group-add $\Rightarrow{ }^{\prime} m$ (infixr $\cdot 70$ )

and $M \quad::{ }^{\prime} m$ set

and smult' $::{ }^{\prime} r \Rightarrow{ }^{\prime} n:: a b$-group-add $\Rightarrow{ }^{\prime} n($ infixr $\star 70)$

and $T \quad::{ }^{\prime} m \Rightarrow{ }^{\prime} n$

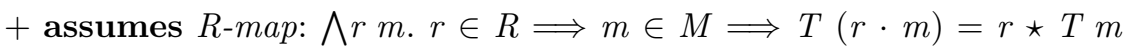

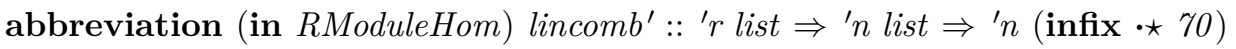

where lincomb' $\equiv$ Codomain.lincomb

lemma (in RModule) RModuleHomI :

assumes GroupHom $M T$

$$
\bigwedge r m . r \in R \Longrightarrow m \in M \Longrightarrow T(r \cdot m)=\operatorname{smult}^{\prime} r(T m)
$$

shows RModuleHom $R$ smult $M$ smult' $T$

$\langle$ proof $\rangle$

locale $R$ ModuleEnd $=$ RModuleHom $R$ smult $M$ smult $T$

for $R \quad::{ }^{\prime} r::$ ring-1 set

and smult $:{ }^{\prime} r \Rightarrow{ }^{\prime} m:: a b$-group-add $\Rightarrow{ }^{\prime} m$ (infixr . 70)

and $M \quad::{ }^{\prime} m$ set

and $T \quad:{ }^{\prime} m \Rightarrow{ }^{\prime} m$

+ assumes endomorph: $\operatorname{Im} G \subseteq M$

locale ModuleHom $=$ RModuleHom UNIV smult $M$ smult' $T$

for smult :: 'r::ring-1 $\Rightarrow$ 'm::ab-group-add $\Rightarrow{ }^{\prime} m$ (infixr · 70)

and $M \quad::{ }^{\prime} m$ set

and smult' $:$ ' $^{\prime} \Rightarrow{ }^{\prime} n:: a b$-group-add $\Rightarrow{ }^{\prime} n($ infixr $\star 70)$

and $T \quad::{ }^{\prime} m \Rightarrow{ }^{\prime} n$

lemmas (in ModuleHom) hom = hom

lemmas (in Module) ModuleHomI = RModuleHomI[THEN ModuleHom.intro]

locale ModuleEnd $=$ ModuleHom smult $M$ smult $T$

for smult :: 'r::ring-1 $\Rightarrow$ 'm::ab-group-add $\Rightarrow{ }^{\prime} m$ (infixr · 70)

and $M \quad::{ }^{\prime} m$ set and $T::{ }^{\prime} m \Rightarrow{ }^{\prime} m$

+ assumes endomorph: $\operatorname{Im} G \subseteq M$

locale RModuleIso $=$ RModuleHom $R$ smult $M$ smult' $T$

for $R \quad:{ }^{\prime} r::$ ring-1 set 


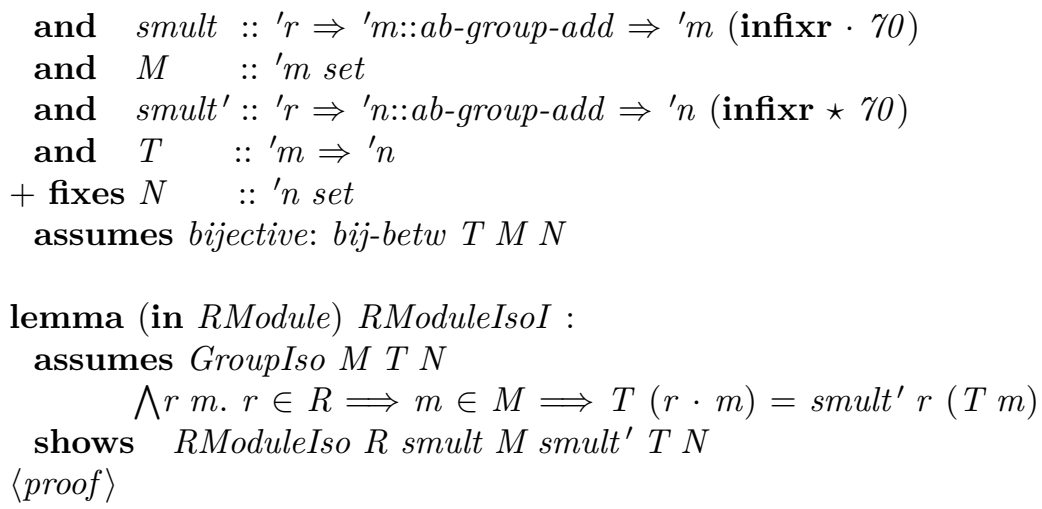

\subsubsection{Basic facts}

lemma (in RModule) trivial-RModuleHom :

$\forall r \in R$. smult' $r 0=0 \Longrightarrow$ RModuleHom $R$ smult $M$ smult' 0 $\langle$ proof $\rangle$

lemma (in RModule) RModHom-idhom : RModuleHom $R$ smult $M$ smult (id $\downarrow M)$ $\langle$ proof $\rangle$

context RModuleHom

begin

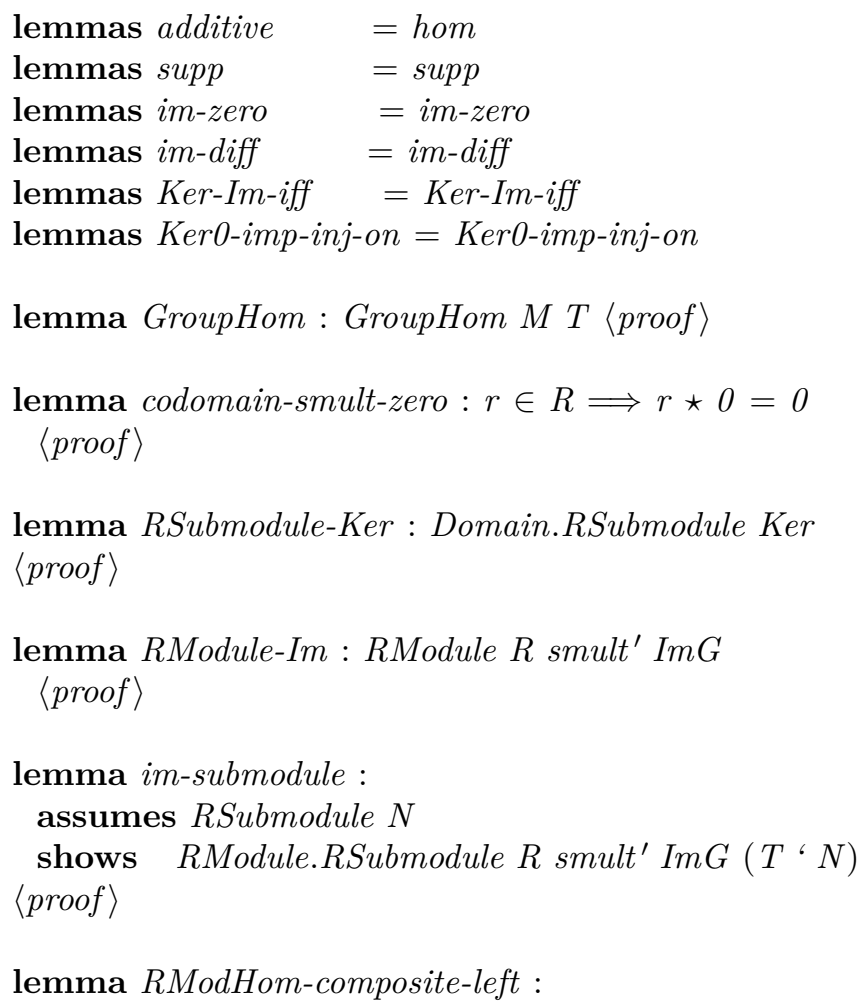

lemma RModHom-composite-left : 
assumes $T$ ' $M \subseteq N$ RModuleHom $R$ smult' $N$ smult" $S$

shows RModuleHom $R$ smult $M$ smult $^{\prime \prime}(S \circ T)$

$\langle$ proof $\rangle$

lemma RModuleHom-restrict0-submodule :

assumes RSubmodule $N$

shows RModuleHom $R$ smult $N$ smult $^{\prime}(T \downarrow N)$

$\langle$ proof $\rangle$

lemma distrib-lincomb :

set $r s \subseteq R \Longrightarrow$ set $m s \subseteq M \Longrightarrow T($ rs $\cdot m s)=r s \cdot \star m a p T m s$

$\langle$ proof $\rangle$

lemma same-image-on-RSpanset-imp-same-hom :

assumes RModuleHom $R$ smult $M$ smult' $S$ set $m s \subseteq M$

$M=$ Domain.R-scalars.RSpan ms $\forall m \in$ set ms. $S m=T m$

shows $S=T$

$\langle$ proof $\rangle$

end

lemma RSubmodule-eigenspace :

fixes smult :: 'r::ring-1 $\Rightarrow$ 'm::ab-group-add $\Rightarrow{ }^{\prime} m$ (infixr · 70)

assumes RModHom: RModuleHom $R$ smult $M$ smult $T$

and $\quad r: r \in R \bigwedge s m . s \in R \Longrightarrow m \in M \Longrightarrow s \cdot r \cdot m=r \cdot s \cdot m$

defines $E: E \equiv\{m \in M$. T $m=r \cdot m\}$

shows RModule.RSubmodule $R$ smult $M E$

$\langle$ proof $\rangle$

\subsubsection{Basic facts about endomorphisms}

lemma (in RModule) Rmap-endomorph-is-RModuleEnd :

assumes grpend: GroupEnd $M T$

and $\quad$ Rmap : ^rm. $r \in R \Longrightarrow m \in M \Longrightarrow T(r \cdot m)=r \cdot(T m)$

shows RModuleEnd $R$ smult $M T$

$\langle$ proof $\rangle$

lemma (in RModuleEnd) GroupEnd : GroupEnd $M T$

$\langle$ proof $\rangle$

lemmas (in RModuleEnd) proj-decomp = GroupEnd.proj-decomp[OF GroupEnd $]$

lemma (in ModuleEnd) RModuleEnd : RModuleEnd UNIV smult MT $\langle$ proof $\rangle$

lemmas (in ModuleEnd) GroupEnd = RModuleEnd.GroupEnd $[$ OF RModuleEnd $]$

lemma RModuleEnd-over-UNIV-is-ModuleEnd :

RModuleEnd UNIV rsmult $M T \Longrightarrow$ ModuleEnd rsmult $M T$ 
$\langle$ proof $\rangle$

\subsubsection{Basic facts about isomorphisms}

context RModuleIso

begin

abbreviation $i n v T \equiv($ the-inv-into $M T) \downarrow N$

lemma GroupIso : GroupIso M T N

$\langle$ proof $\rangle$

lemmas $\operatorname{Im} G \quad=$ GroupIso.ImG $\quad[$ OF GroupIso]

lemmas GroupHom-inv = GroupIso.inv $\quad$ [OF GroupIso]

lemmas invT-into = GroupIso.invT-into [OF GroupIso]

lemmas $T$-invT = GroupIso.T-invT [OF GroupIso]

lemmas invT-eq = GroupIso.invT-eq [OF GroupIso]

lemma RModule $N$ : RModule $R$ smult $^{\prime} N\langle$ proof $\rangle$

lemma inv : RModuleIso $R$ smult' $N$ smult invT $M$

$\langle$ proof $\rangle$

end

\subsection{Inner direct sums of RModules}

lemma (in RModule) RModule-inner-dirsum-el-decomp-Rsmult : assumes $\forall N \in$ set $N$ s. RSubmodule $N$ add-independentS $N s \quad r \in R$ $x \in(\bigoplus N \leftarrow N s . N)$

shows $\quad(\bigoplus N s \leftarrow(r \cdot x))=[r \cdot m . m \leftarrow(\bigoplus N s \leftarrow x)]$ $\langle$ proof $\rangle$

lemma (in RModule) RModuleEnd-inner-dirsum-el-decomp-nth :

assumes $\forall N \in$ set Ns. RSubmodule $N$ add-independent $S$ Ns $n<$ length $N s$ shows $\quad R M o d u l e E n d R$ smult $(\bigoplus N \leftarrow N s . N)(\bigoplus N s \downarrow n)$ $\langle$ proof $\rangle$

\section{Vector Spaces}

\subsection{Locales and basic facts}

Here we don't care about being able to switch scalars.

locale fscalar-mult $=$ scalar-mult smult

for smult :: 'f::field $\Rightarrow{ }^{\prime} v:: a b$-group-add $\Rightarrow{ }^{\prime} v$ (infixr - 70)

abbreviation (in fscalar-mult) findim $\equiv$ fingen

locale VectorSpace $=$ Module smult $V$ 
for smult :: 'f::field $\Rightarrow{ }^{\prime} v::$ ab-group-add $\Rightarrow{ }^{\prime} v($ infixr $\cdot 70)$ and $V \quad::{ }^{\prime} v$ set

lemmas VectorSpaceI $=$ ModuleI $[$ THEN VectorSpace.intro $]$

sublocale VectorSpace $<$ fscalar-mult $\langle$ proof $\rangle$

locale FinDimVectorSpace $=$ VectorSpace

+ assumes findim: findim $V$

lemma (in VectorSpace) FinDimVectorSpaceI :

findim $V \Longrightarrow$ FinDimVectorSpace $(\cdot) \mathrm{V}$

$\langle$ proof $\rangle$

context VectorSpace

begin

abbreviation Subspace :: 'v set $\Rightarrow$ bool where Subspace $\equiv$ Submodule

lemma SubspaceD1 : Subspace $U \Longrightarrow$ VectorSpace smult $U$ $\langle$ proof $\rangle$

lemmas AbGroup

lemmas add-closed

lemmas smult-closed

lemmas one-smult

lemmas smult-assoc

lemmas smult-distrib-left

lemmas smult-distrib-right

lemmas zero-closed

lemmas zero-smult

lemmas smult-zero

lemmas smult-lincomb

lemmas smult-distrib-left-diff

lemmas smult-sum-distrib

lemmas sum-smult-distrib

lemmas lincomb-sum

lemmas lincomb-closed

lemmas lincomb-concat

lemmas lincomb-replicate0-left

lemmas delta-scalars-lincomb-eq-nth

lemmas SpanI

lemmas Span-closed

lemmas SpanD-lincomb-arb-len-coeffs

lemmas SpanI-lincomb-arb-len-coeffs

lemmas in-Span-obtain-same-length-coeffs $=$ in-Span-obtain-same-length-coeffs

lemmas SubspaceI

lemmas subspace-finrank

$$
\begin{aligned}
& =\text { AbGroup } \\
& =\text { add-closed } \\
& =\text { smult-closed } \\
& =\text { one-smult }
\end{aligned}
$$

$=$ smult-assoc

$=$ smult-distrib-left

$=$ smult-distrib-right

$=$ zero-closed

= zero-smult

$=$ smult-zero

$=$ smult-lincomb

$=$ smult-distrib-left-diff

$=$ smult-sum-distrib

= sum-smult-distrib

$=$ lincomb-sum

$=$ lincomb-closed

$=$ lincomb-concat

$=$ lincomb-replicate0-left

$=$ delta-scalars-lincomb-eq-nth

$=$ SpanI

$=$ Span-closed

$=$ SpanD-lincomb-arb-len-coeffs

= SpanI-lincomb-arb-len-coeffs

$=$ SubmoduleI

= submodule-finrank 
lemma cancel-scalar: $\llbracket a \neq 0 ; u \in V ; v \in V ; a \cdot u=a \cdot v \rrbracket \Longrightarrow u=v$ $\langle$ proof $\rangle$

end

\subsection{Linear algebra in vector spaces}

\subsubsection{Linear independence and spanning}

context VectorSpace

begin

$\begin{array}{ll}\text { lemmas Subspace-Span } & =\text { Submodule-Span } \\ \text { lemmas lin-independent-Nil } & =R \text {-lin-independent-Nil } \\ \text { lemmas lin-independentI-concat-all-scalars } & =R \text {-lin-independentI-concat-all-scalars } \\ \text { lemmas lin-independentD-all-scalars } & =R \text {-lin-independentD-all-scalars }\end{array}$

lemmas lin-independent-obtain-unique-scalars $=R$-lin-independent-obtain-unique-scalars

lemma lincomb-Cons-0-imp-in-Span :

$\llbracket v \in V ;$ set $v s \subseteq V ; a \neq 0 ;(a \# a s) \cdot .(v \# v s)=0 \rrbracket \Longrightarrow v \in$ Span vs $\langle$ proof $\rangle$

lemma lin-independent-Cons-conditions :

$\llbracket v \in V$; set $v s \subseteq V ; v \notin$ Span vs; lin-independent vs 》

$\langle$ proof $\rangle$

$\Longrightarrow$ lin-independent $(v \# v s)$

lemma coeff-n0-imp-in-Span-others :

assumes $v \in V$ set us $\subseteq V$ set $v s \subseteq V b \neq 0$ length as $=$ length us $w=(a s @ b \# b s) \cdot .(u s @ v \# v s)$

shows $v \in \operatorname{Span}(w \# u s @ v s)$

$\langle$ proof $\rangle$

lemma lin-independent-replace1-by-lincomb :

assumes set $u s \subseteq V v \in V$ set $v s \subseteq V$ lin-independent (us @v\#vs)

length as $=$ length us $b \neq 0$

shows lin-independent ( ((as@b\#bs).. (us@v\#vs)) \#us@vs )

$\langle$ proof $\rangle$

lemma build-lin-independent-seq :

assumes us- $V$ : set us $\subseteq V$

and indep-us: lin-independent us

shows $\exists$ ws. set ws $\subseteq V \wedge$ lin-independent (ws @ us) $\wedge($ Span $(w s @ u s)=V$

$\langle$ proof $\rangle$

$\vee$ length $w s=n$ )

end 


\subsubsection{Basis for a vector space: basis-for}

abbreviation (in fscalar-mult) basis-for :: $v$ set $\Rightarrow$ 'v list $\Rightarrow$ bool where basis-for $V$ vs $\equiv($ set $v s \subseteq V \wedge V=$ Span vs $\wedge$ lin-independent vs $)$

context VectorSpace

begin

lemma spanset-contains-basis :

set vs $\subseteq V \Longrightarrow \exists$ us. set $u s \subseteq$ set vs $\wedge$ basis-for (Span vs) us $\langle$ proof $\rangle$

lemma basis-for-Span-ex : set vs $\subseteq V \Longrightarrow \exists$ us. basis-for (Span vs) us $\langle$ proof $\rangle$

lemma replace-basis-one-step :

assumes closed: set $v s \subseteq V$ set $u s \subseteq V$ and indep: lin-independent (us@vs)

and $\quad n e w-w: w \in \operatorname{Span}(u s @ v s)-\operatorname{Span} u s$

shows $\exists x s y y s . v s=x s @ y \# y s$

$\langle$ proof $\rangle$

$\wedge$ basis-for (Span (us@vs)) (w\#us@xs@ys)

lemma replace-basis :

assumes closed: set $v s \subseteq V$ and indep-vs: lin-independent vs

shows $\llbracket$ length us $\leq$ length vs; set us $\subseteq$ Span vs; lin-independent us $\rrbracket$

$\Longrightarrow \exists$ pvs. length pvs $=$ length $v s \wedge$ set pvs $=$ set vs

$\langle$ proof $\rangle$

$\wedge$ basis-for (Spanvs) (take (lengthvs) (us @ pvs))

lemma replace-basis-completely :

$\llbracket$ set $v s \subseteq V$; lin-independent vs; length us = length vs;

set us $\subseteq$ Span vs; lin-independent us $\rrbracket \Longrightarrow$ basis-for (Span vs) us $\langle$ proof $\rangle$

lemma basis-for-obtain-unique-scalars :

basis-for $V$ vs $\Longrightarrow v \in V \Longrightarrow \exists$ ! as. length as $=$ length $v s \wedge v=a s \cdot v$ s $\langle$ proof $\rangle$

lemma add-unique-scalars :

assumes vs: basis-for $V$ vs and $v: v \in V$ and $v^{\prime}: v^{\prime} \in V$

defines as: as $\equiv(T H E$ ds. length $d s=$ length $v s \wedge v=d s \cdot v s)$

and $\quad b s: b s \equiv\left(T H E d s\right.$. length $d s=$ length $\left.v s \wedge v^{\prime}=d s \cdot v s\right)$

and $\quad c s: c s \equiv\left(T H E d s\right.$. length $d s=$ length $\left.v s \wedge v+v^{\prime}=d s \cdot v s\right)$

shows $\quad c s=[a+b .(a, b) \leftarrow z i p$ as $b s]$

$\langle$ proof $\rangle$

lemma smult-unique-scalars :

fixes $a::^{\prime} f$

assumes vs: basis-for $V$ vs and $v: v \in V$

defines as: as $\equiv(T H E$ cs. length $c s=$ length $v s \wedge v=c s \cdot v s)$ 


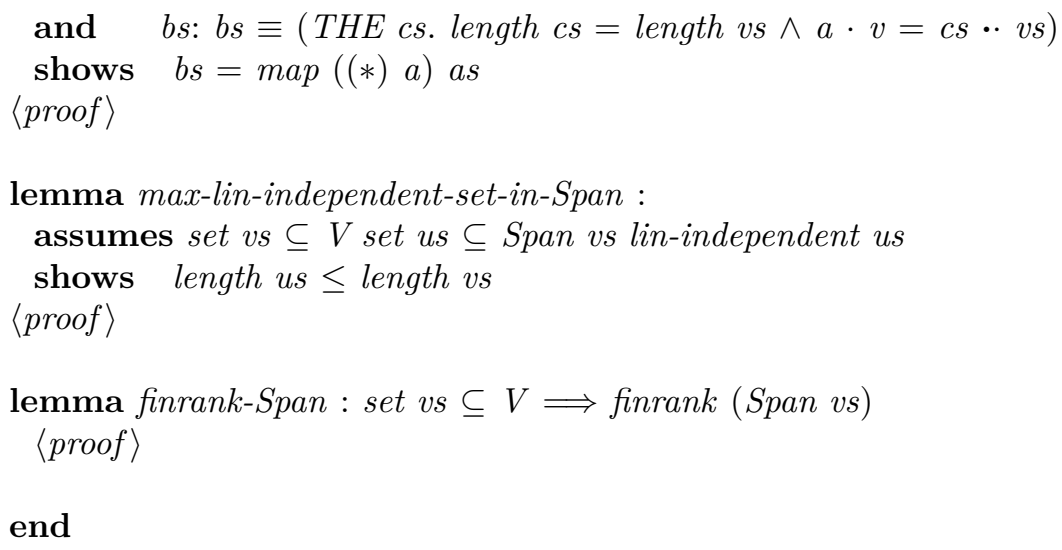

\subsection{Finite dimensional spaces}

context VectorSpace

begin

lemma dim-eq-size-basis : basis-for $V$ vs $\Longrightarrow$ length vs $=\operatorname{dim} V$ $\langle$ proof $\rangle$

lemma finrank-imp-findim : assumes finrank $V$ shows findim $V$ $\langle$ proof $\rangle$

lemma subspace-Span-is-findim :

$\llbracket$ set vs $\subseteq V ;$ Subspace $W ; W \subseteq$ Span vs $\rrbracket \Longrightarrow$ findim $W$ $\langle$ proof $\rangle$

end

context FinDim VectorSpace

begin

lemma Subspace-is-findim : Subspace $U \Longrightarrow$ findim $U$ $\langle$ proof $\rangle$

lemma basis-ex : $\exists$ vs. basis-for $V$ vs $\langle$ proof $\rangle$

lemma lin-independent-length-le-dim : set $u s \subseteq V \Longrightarrow$ lin-independent us $\Longrightarrow$ length us $\leq \operatorname{dim} V$ $\langle$ proof $\rangle$

lemma too-long-lin-dependent : set us $\subseteq V \Longrightarrow$ length us $>\operatorname{dim} V \Longrightarrow \neg$ lin-independent us $\langle$ proof $\rangle$ 




\subsection{Vector space homomorphisms}

\subsubsection{Locales}

locale VectorSpaceHom $=$ ModuleHom smult $V$ smult ${ }^{\prime} T$

for smult :: 'f::field $\Rightarrow$ 'v::ab-group-add $\Rightarrow{ }^{\prime} v$ (infixr · 70)

and $V \quad::{ }^{\prime} v$ set

and smult' :: 'f $\Rightarrow{ }^{\prime} w:: a b-g r o u p-a d d \Rightarrow{ }^{\prime} w($ infixr $\star 70)$

and $T \quad::{ }^{\prime} v \Rightarrow{ }^{\prime} w$

sublocale VectorSpaceHom < VectorSpace $\langle$ proof $\rangle$

lemmas (in VectorSpace)

VectorSpaceHomI = ModuleHomI[THEN VectorSpaceHom.intro $]$

lemma (in VectorSpace) VectorSpaceHomI-fromaxioms :

assumes $\bigwedge g g^{\prime} . g \in V \Longrightarrow g^{\prime} \in V \Longrightarrow T\left(g+g^{\prime}\right)=T g+T g^{\prime}$ supp $T \subseteq V$

$\bigwedge r m . r \in U N I V \Longrightarrow m \in V \Longrightarrow T(r \cdot m)=\operatorname{smult}^{\prime} r(T m)$

shows VectorSpaceHom smult $V$ smult' $T$ 
$\langle$ proof $\rangle$

locale VectorSpaceEnd $=$ VectorSpaceHom smult $V$ smult $T$

for smult :: 'f::field $\Rightarrow{ }^{\prime} v:: a b$-group-add $\Rightarrow{ }^{\prime} v$ (infixr - 70)

and $V \quad::{ }^{\prime} v$ set

and $T \quad::^{\prime} v \Rightarrow{ }^{\prime} v$

+ assumes endomorph: $\operatorname{Im} G \subseteq V$

abbreviation (in VectorSpace) VEnd $\equiv$ VectorSpaceEnd smult $V$

lemma VectorSpaceEndI :

fixes smult :: 'f::field $\Rightarrow$ ' $v::$ ab-group-add $\Rightarrow{ }^{\prime} v$

assumes VectorSpaceHom smult $V$ smult $T T^{\prime} V \subseteq V$

shows VectorSpaceEnd smult $V T$

$\langle$ proof $\rangle$

lemma (in VectorSpaceEnd) VectorSpaceHom: VectorSpaceHom smult $V$ smult $T$ $\langle$ proof $\rangle$

lemma (in VectorSpaceEnd) ModuleEnd : ModuleEnd smult V T $\langle$ proof $\rangle$

locale VectorSpaceIso $=$ VectorSpaceHom smult $V$ smult ${ }^{\prime} T$

for smult $::{ }^{\prime} f::$ field $\Rightarrow{ }^{\prime} v:: a b$-group-add $\Rightarrow^{\prime} v$ (infixr $\left.\cdot 70\right)$

and $V \quad::{ }^{\prime} v$ set

and smult' :: 'f $\Rightarrow{ }^{\prime} w:: a b$-group-add $\Rightarrow{ }^{\prime} w(\mathbf{i n f i x r} \star 70)$

and $T \quad::^{\prime} v \Rightarrow{ }^{\prime} w$

+ fixes $W \quad::{ }^{\prime} w$ set

assumes bijective: bij-betw $T V W$

abbreviation (in VectorSpace) isomorphic ::

$\left(' f \Rightarrow\right.$ 'w::ab-group-add $\left.\Rightarrow{ }^{\prime} w\right) \Rightarrow{ }^{\prime} w$ set $\Rightarrow$ bool

where isomorphic smult ${ }^{\prime} W \equiv\left(\exists T\right.$. VectorSpaceIso smult $V$ smult $\left.{ }^{\prime} T W\right)$

\subsubsection{Basic facts}

lemma (in VectorSpace) trivial-VectorSpaceHom :

$(\bigwedge a$. smult' a $0=0) \Longrightarrow$ VectorSpaceHom smult $V$ smult ${ }^{\prime} 0$

$\langle$ proof $\rangle$

lemma (in VectorSpace) VectorSpaceHom-idhom :

VectorSpaceHom smult $V$ smult $(i d \downarrow V)$

$\langle$ proof $\rangle$

context VectorSpaceHom

begin

$\begin{array}{ll}\text { lemmas hom } & =\text { hom } \\ \text { lemmas supp } & =\text { supp }\end{array}$ 




lemma VectorSpace-Im : VectorSpace smult' ImG $\langle$ proof $\rangle$

lemma VectorSpaceHom-scalar-mul :

VectorSpaceHom smult $V$ smult $^{\prime}(\lambda v . a \star T v)$ $\langle$ proof $\rangle$

lemma VectorSpaceHom-composite-left : assumes $\operatorname{Im} G \subseteq W$ VectorSpaceHom smult' $W$ smult" $S$ shows VectorSpaceHom smult $V$ smult $^{\prime \prime}(S \circ T)$ $\langle$ proof $\rangle$

lemma findim-domain-findim-image : assumes findim $V$ shows fscalar-mult.findim smult' ImG $\langle$ proof $\rangle$

end

lemma (in VectorSpace) basis-im-defines-hom :

fixes smult' :: $f \Rightarrow{ }^{\prime} w:: a b$-group-add $\Rightarrow$ 'w (infixr $\left.\star 70\right)$

and lincomb' :: 'f list $\Rightarrow$ 'w list $\Rightarrow{ }^{\prime} w($ infixr $• \star 70)$

defines lincomb' $b^{\prime}$ lincomb $b^{\prime} \equiv$ scalar-mult.lincomb smult ${ }^{\prime}$ assumes $V S p W$ : VectorSpace smult' $W$ and basis $V$ : basis-for $V$ vs and basis $V$-im : set $w s \subseteq W$ length ws = length vs shows $\exists$ ! T. VectorSpaceHom smult $V$ smult $T \wedge$ map $T$ vs $=$ ws $\langle$ proof $\rangle$

\subsubsection{Hom-sets}

definition VectorSpaceHomSet ::

$\left(' f::\right.$ field $\Rightarrow{ }^{\prime} v:: a b$-group-add $\left.\Rightarrow{ }^{\prime} v\right) \Rightarrow{ }^{\prime} v$ set $\Rightarrow\left({ }^{\prime} f \Rightarrow{ }^{\prime} w:: a b\right.$-group-add $\left.\Rightarrow{ }^{\prime} w\right)$ $\Rightarrow{ }^{\prime} w$ set $\Rightarrow\left({ }^{\prime} v \Rightarrow{ }^{\prime} w\right)$ set

where VectorSpaceHomSet fsmult $V$ fsmult' $W$ $\equiv\{T$. VectorSpaceHom fsmult $V$ fsmult' $T\} \cap\left\{T . T{ }^{\prime} V \subseteq W\right\}$ 
abbreviation (in VectorSpace) VectorSpaceEndSet $\equiv\{S$. VEnd $S\}$

lemma VectorSpaceHomSetI :

VectorSpaceHom fsmult $V$ fsmult' $T \Longrightarrow T$ ' $V \subseteq W$ $\langle$ proof $\rangle$ $\Longrightarrow T \in$ VectorSpaceHomSet fsmult $V$ fsmult ${ }^{\prime} W$

lemma VectorSpaceHomSetD-VectorSpaceHom :

$T \in$ VectorSpaceHomSet fsmult $V$ fsmult ${ }^{\prime} N$

$\Longrightarrow$ VectorSpaceHom fsmult $V$ fsmult' $T$

$\langle$ proof $\rangle$

lemma VectorSpaceHomSetD-Im :

$T \in$ VectorSpaceHomSet fsmult $V$ fsmult' $W \Longrightarrow T^{\prime} V \subseteq W$

$\langle$ proof $\rangle$

context VectorSpace

begin

lemma VectorSpaceHomSet-is-fmaps-in-GroupHomSet :

fixes smult':: 'f $\Rightarrow$ 'w::ab-group-add $\Rightarrow$ 'w (infixr $\star 70)$

shows VectorSpaceHomSet smult $V$ smult' $W$

$=($ GroupHomSet $V W) \cap\{T . \forall a . \forall v \in V . T(a \cdot v)=a \star(T v)\}$

$\langle$ proof $\rangle$

lemma Group-VectorSpaceHomSet :

fixes smult':: 'f $\Rightarrow$ 'w::ab-group-add $\Rightarrow{ }^{\prime} w(\mathbf{i n f i x r} \star 70)$

assumes VectorSpace smult' $W$

shows Group (VectorSpaceHomSet smult $V$ smult' $W$ )

$\langle$ proof $\rangle$

lemma VectorSpace-VectorSpaceHomSet :

fixes smult' :: $f \Rightarrow$ 'w::ab-group-add $\Rightarrow$ 'w (infixr $\star 70)$

and hom-smult :: 'f $\Rightarrow\left({ }^{\prime} v \Rightarrow{ }^{\prime} w\right) \Rightarrow\left({ }^{\prime} v \Rightarrow{ }^{\prime} w\right)$ (infixr $\left.\star \cdot 70\right)$

defines hom-smult: hom-smult $\equiv \lambda a T v . a \star T v$

assumes VSpW: VectorSpace smult' $W$

shows VectorSpace hom-smult (VectorSpaceHomSet smult V smult' $W$ ) $\langle$ proof $\rangle$

end

\subsubsection{Basic facts about endomorphisms}

lemma ModuleEnd-over-field-is-VectorSpaceEnd :

fixes smult :: 'f::field $\Rightarrow$ 'v::ab-group-add $\Rightarrow{ }^{\prime} v$ assumes ModuleEnd smult $V T$

shows VectorSpaceEnd smult $V T$

$\langle$ proof $\rangle$ 
context VectorSpace

begin

lemmas VectorSpaceEnd-inner-dirsum-el-decomp-nth $=$

RModuleEnd-inner-dirsum-el-decomp-nth [

THEN RModuleEnd-over-UNIV-is-ModuleEnd,

THEN ModuleEnd-over-field-is-VectorSpaceEnd

]

abbreviation end-smult :: 'f $\Rightarrow\left({ }^{\prime} v \Rightarrow{ }^{\prime} v\right) \Rightarrow\left({ }^{\prime} v \Rightarrow{ }^{\prime} v\right)$ (infixr .. 70)

where $a \cdot T \equiv(\lambda v \cdot a \cdot T v)$

abbreviation end-lincomb

: 'f list $\Rightarrow\left(\left({ }^{\prime} v \Rightarrow{ }^{\prime} v\right)\right.$ list $) \Rightarrow\left({ }^{\prime} v \Rightarrow{ }^{\prime} v\right)($ infixr $\cdots 70)$

where end-lincomb $\equiv$ scalar-mult.lincomb end-smult

lemma end-smulto: $a \cdot \cdot 0=0$

$\langle$ proof $\rangle$

lemma end-0smult: range $T \subseteq V \Longrightarrow 0 \quad . \cdot T=0$

$\langle$ proof $\rangle$

lemma end-smult-distrib-left :

assumes range $S \subseteq V$ range $T \subseteq V$

shows $\quad a \cdot \cdot(S+T)=a \cdot \cdot S+a \cdot \cdot T$

$\langle$ proof $\rangle$

lemma end-smult-distrib-right :

assumes range $T \subseteq V$

shows $(a+b) \cdot T=a \cdot T+b \cdot \cdot T$

$\langle$ proof $\rangle$

lemma end-smult-assoc :

assumes range $T \subseteq V$

shows $a \cdot b \cdot \cdot \cdot T=(a * b) \cdot \cdot T$

$\langle$ proof $\rangle$

lemma end-smult-comp-comm-left $:(a \cdot \cdot T) \circ S=a \cdot \cdot(T \circ S)$

$\langle$ proof $\rangle$

lemma end-idhom : VEnd $(i d \downarrow V)$

$\langle$ proof $\rangle$

lemma VectorSpaceEndSet-is-VectorSpaceHomSet :

VectorSpaceHomSet smult $V$ smult $V=\{T$. VEnd $T\}$

$\langle$ proof $\rangle$

lemma VectorSpace-VectorSpaceEndSet : VectorSpace end-smult VectorSpaceEndSet 


$$
\langle\text { proof }\rangle
$$

end

context VectorSpaceEnd

begin

lemmas $f$-map

$=R-m a p$

lemmas supp

$=\operatorname{supp}$

lemmas GroupEnd

lemmas idhom-left $=$ ModuleEnd.GroupEnd $[$ OF ModuleEnd $]$

lemmas range

$=$ idhom-left

lemmas Ker0-imp-inj-on

$=$ GroupEnd.range $[$ OF GroupEnd $]$

$=$ Ker0-imp-inj-on

lemmas inj-on-imp-Ker0

$=i n j$-on-imp-Ker0

lemmas nonzero-Ker-el-imp-n-inj = nonzero-Ker-el-imp-n-inj

lemmas VectorSpaceHom-composite-left

$=$ VectorSpaceHom-composite-left $[$ OF endomorph $]$

lemma in-VEndSet : $T \in$ VectorSpaceEndSet

$\langle$ proof $\rangle$

lemma end-smult-comp-comm-right :

range $S \subseteq V \Longrightarrow T \circ(a \cdot S)=a \cdot \cdot(T \circ S)$

$\langle$ proof $\rangle$

lemma VEnd-end-smult-VEnd : VEnd $(a \cdots T)$

$\langle$ proof $\rangle$

lemma VEnd-composite-left :

assumes $V E n d S$

shows $\operatorname{VEnd}(S \circ T)$

$\langle$ proof $\rangle$

lemma VEnd-composite-right : VEnd $S \Longrightarrow$ VEnd $(T \circ S)$

$\langle$ proof $\rangle$

end

lemma (in VectorSpace) inj-comp-end :

assumes VEnd $S$ inj-on $S V$ VEnd $T$ inj-on $T V$

shows inj-on $(S \circ T) V$

$\langle$ proof $\rangle$

lemma (in VectorSpace) $n$-inj-comp-end :

$\llbracket V E n d S ; \operatorname{VEnd} T ; \neg$ inj-on $(S \circ T) V \rrbracket \Longrightarrow \neg$ inj-on $S V \vee \neg i n j$-on $T V$ $\langle$ proof $\rangle$ 


\subsubsection{Polynomials of endomorphisms}

context VectorSpaceEnd

begin

primrec endpow :: nat $\Rightarrow\left({ }^{\prime} v \Rightarrow^{\prime} v\right)$

where endpow0: endpow $0=i d \downarrow V$

endpowSuc: endpow $($ Suc $n)=T \circ($ endpow $n)$

definition polymap :: 'f poly $\Rightarrow\left({ }^{\prime} v \Rightarrow^{\prime} v\right)$

where polymap $p \equiv($ coeffs $p) \cdots($ map endpow $[0 . .<$ Suc $($ degree $p)])$

lemma VEnd-endpow : VEnd (endpow $n)$

$\langle$ proof $\rangle$

lemma endpow-list-apply-closed :

$v \in V \Longrightarrow \operatorname{set}(\operatorname{map}(\lambda S . S v)($ map endpow $[0 . .<k])) \subseteq V$

$\langle$ proof $\rangle$

lemma map-endpow-Suc:

map endpow $[0 . .<S u c n]=(i d \downarrow V) \# \operatorname{map}((\circ) T)($ map endpow $[0 . .<n])$

$\langle$ proof $\rangle$

lemma T-endpow-list-apply-commute :

map $T(\operatorname{map}(\lambda S . S v)(\operatorname{map}$ endpow $[0 . .<n]))$

$\langle$ proof $\rangle$

$=\operatorname{map}(\lambda S . S v)(\operatorname{map}((\circ) T)(\operatorname{map}$ endpow $[0 . .<n]))$

lemma polymap0 : polymap $0=0$

$\langle$ proof $\rangle$

lemma VEnd-polymap : VEnd (polymap $p)$

$\langle$ proof $\rangle$

lemma polymap-pCons : polymap $($ pCons a $p)=a \cdot(i d \downarrow V)+(T \circ($ polymap $p))$ $\langle$ proof $\rangle$

lemma polymap-plus : polymap $(p+q)=$ polymap $p+$ polymap $q$

$\langle$ proof $\rangle$

lemma polymap-polysmult : polymap (Polynomial.smult a $p)=a \cdot \cdot$ polymap $p$ $\langle$ proof $\rangle$

lemma polymap-times : polymap $(p * q)=($ polymap $p) \circ($ polymap $q)$ $\langle$ proof $\rangle$

lemma polymap-apply :

assumes $v \in V$

shows polymap $p v=($ coeffs $p)$

.. $(\operatorname{map}(\lambda S . S v)($ map endpow $[0 . .<$ Suc $($ degree $p)]))$ 


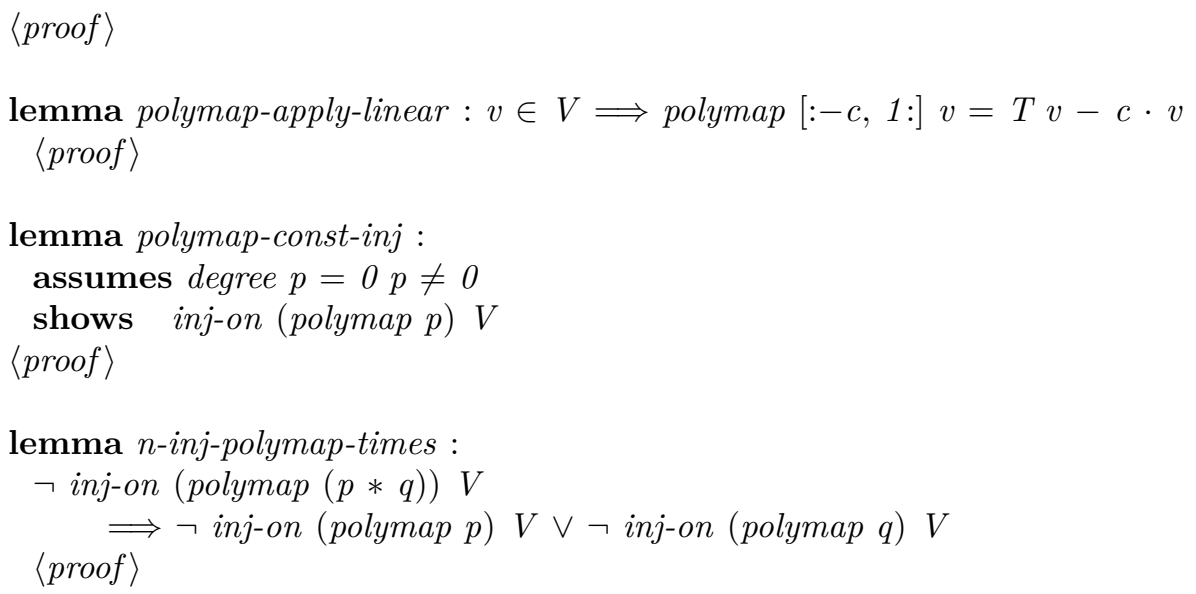

In the following lemma, $\left[:-c, 1:{ }^{\prime} a:\right]$ is the linear polynomial $x-c$.

lemma $n$-inj-polymap-findlinear :

assumes alg-closed: $\bigwedge p$ ::'f poly. degree $p>0 \Longrightarrow \exists$ c. poly $p c=0$

shows $\quad p \neq 0 \Longrightarrow \neg i n j$-on (polymap $p) V$

$\langle$ proof $\rangle$ $\Longrightarrow \exists c . \neg$ inj-on (polymap [:-c, $1:]) V$

end

\subsubsection{Existence of eigenvectors of endomorphisms of finite-dimensional vector spaces}

lemma (in FinDimVectorSpace) endomorph-has-eigenvector : assumes alg-closed: $\bigwedge p$ ::'a poly. degree $p>0 \Longrightarrow \exists$ c. poly $p c=0$

and $\quad \operatorname{dim} \quad \operatorname{dim} V>0$

and endo : VectorSpaceEnd smult $V T$

shows $\exists c u . u \in V \wedge u \neq 0 \wedge T u=c \cdot u$

$\langle$ proof $\rangle$

\section{Modules Over a Group Ring}

\subsection{Almost-everywhere-zero functions as scalars}

locale aezfun-scalar-mult = scalar-mult smult for smult ::

$\left({ }^{\prime} r:\right.$ ring-1, 'g::group-add) aezfun $\Rightarrow{ }^{\prime} v:: a b$-group-add $\Rightarrow{ }^{\prime} v(\mathbf{i n f i x r} \cdot 70)$ begin

definition fsmult :: $r \Rightarrow{ }^{\prime} v \Rightarrow{ }^{\prime} v$ (infixr $\left.\sharp \cdot 70\right)$ where $a \sharp \cdot v \equiv\left(\begin{array}{lll}a & \delta \delta & 0\end{array}\right) \cdot v$ abbreviation flincomb : ${ }^{\prime} r$ list $\Rightarrow{ }^{\prime} v$ list $\Rightarrow{ }^{\prime} v$ (infixr $\left.\bullet \sharp \cdot 70\right)$

where as $\bullet \sharp \cdot v s \equiv$ scalar-mult.lincomb fsmult as vs abbreviation f-lin-independent : ' $v$ list $\Rightarrow$ bool

where $f$-lin-independent $\equiv$ scalar-mult.lin-independent fsmult abbreviation fSpan :: 'v list $\Rightarrow$ 'v set where fSpan $\equiv$ scalar-mult.Span fsmult 
definition Gmult :: $g \Rightarrow{ }^{\prime} v \Rightarrow{ }^{\prime} v($ infixr $* \cdot 70)$ where $g * \cdot v \equiv\left(\begin{array}{lll}1 & \delta \delta & g\end{array}\right) \cdot v$

lemmas $R$-scalar-mult $=R$-scalar-mult

lemma fsmultD : $a \sharp \cdot v=\left(\begin{array}{lll}a & \delta \delta & 0\end{array}\right) \cdot v$ $\langle$ proof $\rangle$

lemma GmultD : $g * \cdot v=(1 \delta \delta g) \cdot v$

$\langle$ proof $\rangle$

definition negGorbit-list :: 'g list $\Rightarrow\left({ }^{\prime} a \Rightarrow{ }^{\prime} v\right) \Rightarrow{ }^{\prime} a$ list $\Rightarrow{ }^{\prime} v$ list list where negGorbit-list gs $T$ as $\equiv \operatorname{map}(\lambda g$. map $(G m u l t(-g) \circ T)$ as $) g s$

lemma negGorbit-Cons : negGorbit-list ( $g \# g s) T$ as $\langle$ proof $\rangle$ $=($ map $($ Gmult $(-g) \circ T)$ as) \# negGorbit-list gs $T$ as

lemma length-negGorbit-list : length (negGorbit-list gs $T$ as) = length gs $\langle$ proof $\rangle$

lemma length-negGorbit-list-sublist :

$f s \in$ set (negGorbit-list gs $T$ as) $\Longrightarrow$ length $f s=$ length as $\langle$ proof $\rangle$

lemma length-concat-negGorbit-list : length (concat (negGorbit-list gs $T$ as $))=($ length gs $) *($ length as $)$ $\langle$ proof $\rangle$

lemma negGorbit-list-nth :

$\bigwedge i . i<$ length $g s \Longrightarrow($ negGorbit-list gs $T$ as $) ! i=\operatorname{map}($ Gmult $(-g s ! i) \circ T)$ as $\langle$ proof $\rangle$

end

\subsection{Locale and basic facts}

locale FGModule = ActingGroup?: Group G

+ FGMod?: RModule ActingGroup.group-ring smult $V$

for $G \quad::{ }^{\prime} g::$ group-add set

and smult :: ('f::field, 'g) aezfun $\Rightarrow{ }^{\prime} v:: a b$-group-add $\Rightarrow{ }^{\prime} v(\mathbf{i n f i x r} \cdot 70)$

and $V \quad:$ ' $^{\prime} v$ set

sublocale FGModule < aezfun-scalar-mult $\langle$ proof $\rangle$

lemma (in Group) trivial-FGModule :

fixes smult :: ('f::field, ' $g$ ) aezfun $\Rightarrow{ }^{\prime} v:: a b$-group-add $\Rightarrow{ }^{\prime} v$ assumes smult-zero: $\forall$ a $\in$ group-ring. smult $a\left(0::^{\prime} v\right)=0$

shows FGModule G smult (0::'v set) 
$\langle$ proof $\rangle$

context FGModule

begin

abbreviation $F G::\left({ }^{\prime} f, g\right)$ aezfun set where $F G \equiv$ ActingGroup.group-ring abbreviation FGSubmodule $\equiv$ RSubmodule

abbreviation FG-proj $\equiv$ ActingGroup.RG-proj

lemma GroupG: Group $G\langle$ proof $\rangle$

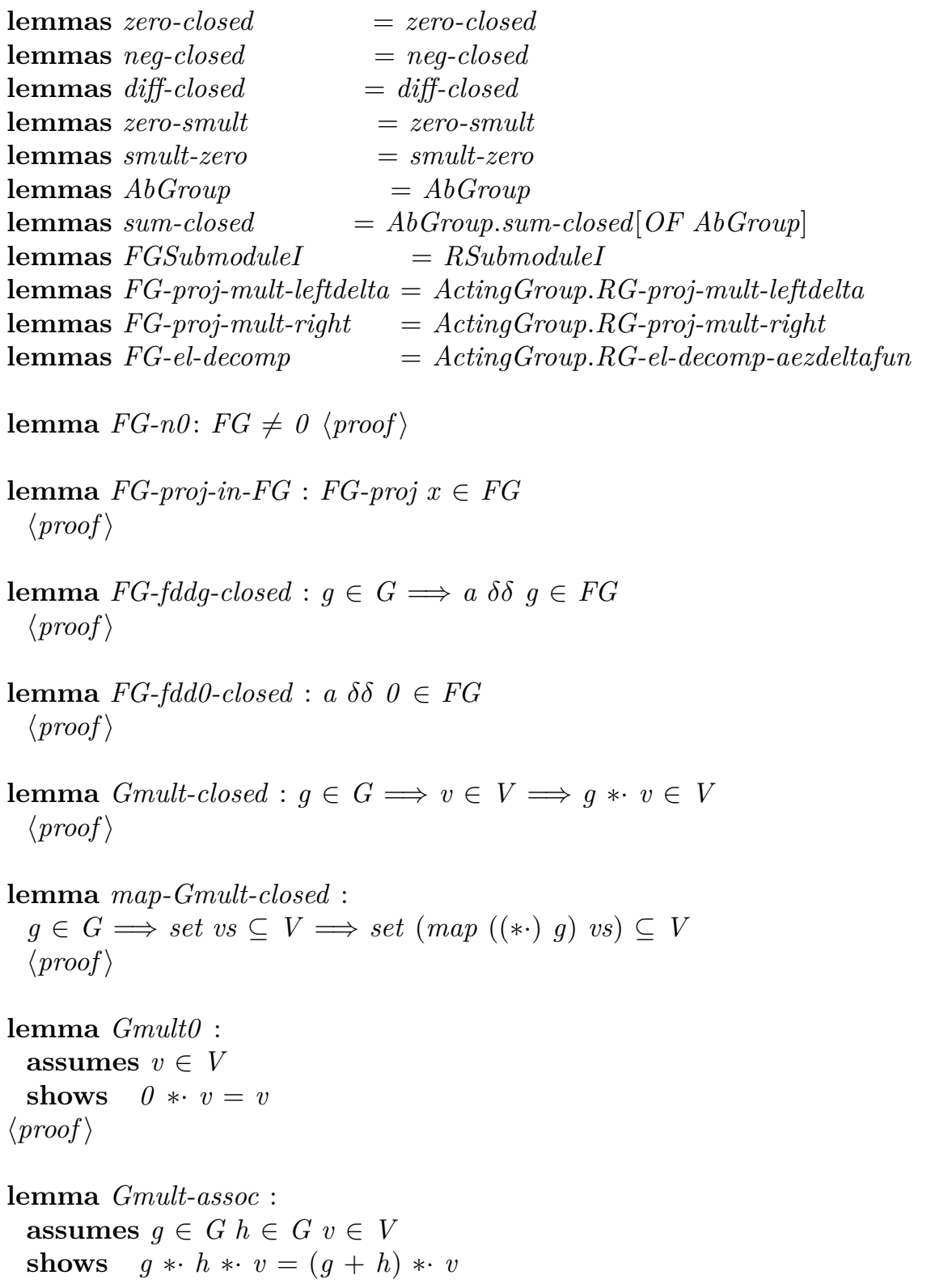


$\langle$ proof $\rangle$

lemma Gmult-distrib-left :

$\llbracket g \in G ; v \in V ; v^{\prime} \in V \rrbracket \Longrightarrow g * \cdot\left(v+v^{\prime}\right)=g * \cdot v+g * \cdot v^{\prime}$ $\langle$ proof $\rangle$

lemma neg-Gmult $: g \in G \Longrightarrow v \in V \Longrightarrow g * \cdot(-v)=-(g * \cdot v)$

$\langle$ proof $\rangle$

lemma Gmult-neg-left $: g \in G \Longrightarrow v \in V \Longrightarrow(-g) * \cdot g * \cdot v=v$ $\langle$ proof $\rangle$

lemma $f d d g$-smult-decomp $: g \in G \Longrightarrow v \in V \Longrightarrow(f \delta \delta g) \cdot v=f \sharp \cdot g * \cdot v$ $\langle$ proof $\rangle$

lemma sum-list-aezdeltafun-smult-distrib :

assumes $v \in V$ set (map snd fgs) $\subseteq G$

shows $\left(\sum(f, g) \leftarrow f g s . f \delta \delta g\right) \cdot v=\left(\sum(f, g) \leftarrow f g s . f \sharp \cdot g * \cdot v\right)$

$\langle$ proof $\rangle$

abbreviation GSubspace $\equiv$ RSubmodule

abbreviation GSpan $\equiv$ RSpan

abbreviation $G$-fingen $\equiv R$-fingen

lemma GSubspaceI : FGModule $G$ smult $U \Longrightarrow U \subseteq V \Longrightarrow$ GSubspace $U$ $\langle$ proof $\rangle$

lemma GSubspace-is-FGModule :

assumes GSubspace $U$

shows FGModule $G$ smult $U$

$\langle$ proof $\rangle$

lemma restriction-to-subgroup-is-module :

fixes $H::$ ' $g$ set

assumes subgrp: Group.Subgroup $G H$

shows FGModule $H$ smult $V$

$\langle$ proof $\rangle$

lemma negGorbit-list- $V$ :

assumes set $g s \subseteq G T$ ' (set as $) \subseteq V$

shows set (concat (negGorbit-list gs $T$ as) $) \subseteq V$

$\langle$ proof $\rangle$

lemma negGorbit-list-Cons0 :

$$
\begin{aligned}
& T \cdot(\text { set as }) \subseteq V \\
& \langle\text { proof }\rangle
\end{aligned} \text { negGorbit-list }(0 \# g s) \text { T as }=(\text { map } T \text { as }) \#(\text { negGorbit-list gs } T \text { as })
$$

end 


\subsection{Modules over a group ring as a vector spaces}

context FGModule

begin

lemma fVectorSpace : VectorSpace fsmult $V$

$\langle$ proof $\rangle$

abbreviation fSubspace $\equiv$ VectorSpace.Subspace fsmult $V$

abbreviation fbasis-for $\equiv$ fscalar-mult.basis-for fsmult

abbreviation fdim $\equiv$ scalar-mult.dim fsmult $V$

lemma VectorSpace-fSubspace : fSubspace $W \Longrightarrow$ VectorSpace fsmult $W$

$\langle$ proof $\rangle$

lemma fsmult-closed : $v \in V \Longrightarrow a \sharp \cdot v \in V$

$\langle$ proof $\rangle$

$\begin{array}{llll}\text { lemmas one-fsmult } & {[\text { simp }]} & =\text { VectorSpace.one-smult } \quad[\text { OF fVectorSpace }] \\ \text { lemmas fsmult-assoc } & {[\text { simp }]} & =\text { VectorSpace.smult-assoc }[\text { OF fVectorSpace }] \\ \text { lemmas fsmult-zero } & {[\text { simp }]} & =\text { VectorSpace.smult-zero }[\text { OF fVectorSpace }]\end{array}$

$\begin{array}{lrl}\text { lemmas fsmult-zero } \quad[\text { simp }] & =\text { VectorSpace.smult-zero }[\text { OF } \\ \text { lemmas fsmult-distrib-left }[\text { simp }] & =\text { VectorSpace.smult-distrib-left }\end{array}$

[OF fVectorSpace]

lemmas flincomb-closed = VectorSpace.lincomb-closed $\quad[$ OF fVectorSpace $]$

lemmas fsmult-sum-distrib = VectorSpace.smult-sum-distrib [OF fVectorSpace]

lemmas sum-fsmult-distrib = VectorSpace.sum-smult-distrib [OF fVectorSpace]

lemmas flincomb-concat = VectorSpace.lincomb-concat $\quad[$ OF fVectorSpace $]$

lemmas fSpan-closed = VectorSpace.Span-closed [OF fVectorSpace $]$

lemmas flin-independentD-all-scalars

$=$ VectorSpace.lin-independentD-all-scalars $[$ OF fVectorSpace $]$

lemmas in-fSpan-obtain-same-length-coeffs

$=$ VectorSpace.in-Span-obtain-same-length-coeffs [OF fVectorSpace $]$

lemma fsmult-smult-comm : $r \in F G \Longrightarrow v \in V \Longrightarrow a \sharp \cdot r \cdot v=r \cdot a \sharp \cdot v$ $\langle$ proof $\rangle$

lemma fsmult-Gmult-comm : $g \in G \Longrightarrow v \in V \Longrightarrow a \sharp \cdot g * \cdot v=g * \cdot a \sharp \cdot v$ $\langle$ proof $\rangle$

lemma Gmult-flincomb-comm :

assumes $g \in G$ set $v s \subseteq V$

shows $g * \cdot a s \cdot \sharp \cdot v s=a s \cdot \sharp \cdot(\operatorname{map}($ Gmult $g) v s)$

$\langle$ proof $\rangle$

lemma GSubspace-is-Subspace :

GSubspace $U \Longrightarrow$ VectorSpace.Subspace fsmult $V U$

$\langle$ proof $\rangle$

end 


\subsection{Homomorphisms of modules over a group ring}

\subsubsection{Locales}

locale FGModuleHom = ActingGroup?: Group G

+ RModHom?: RModuleHom ActingGroup.group-ring smult $V$ smult' $T$

for $G \quad::{ }^{\prime} g:$ :group-add set

and smult :: ('f::field, 'g) aezfun $\Rightarrow{ }^{\prime} v:: a b$-group-add $\Rightarrow{ }^{\prime} v(\mathbf{i n f i x r} \cdot 70)$

and $V \quad:{ }^{\prime} v$ set

and smult':: ('f, 'g) aezfun $\Rightarrow{ }^{\prime} w:: a b$-group-add $\Rightarrow{ }^{\prime} w(\mathbf{i n f i x r} \star 70)$

and $T \quad:{ }^{\prime} v \Rightarrow{ }^{\prime} w$

sublocale FGModuleHom < FGModule $\langle$ proof $\rangle$

lemma (in FGModule) FGModuleHomI-fromaxioms :

assumes $\bigwedge v v^{\prime} . v \in V \Longrightarrow v^{\prime} \in V \Longrightarrow T\left(v+v^{\prime}\right)=T v+T v^{\prime}$

supp $T \subseteq V \wedge r m . r \in F G \Longrightarrow m \in V \Longrightarrow T(r \cdot m)=\operatorname{smult}^{\prime} r(T m)$

shows FGModuleHom $G$ smult $V$ smult' $T$

$\langle$ proof $\rangle$

locale FGModuleEnd = FGModuleHom G smult $V$ smult $T$

for $G \quad::{ }^{\prime} g::$ group-add set

and $F G \quad::($ ' $f::$ field,$' g)$ aezfun set

and smult :: ('f, 'g) aezfun $\Rightarrow{ }^{\prime} v:: a b$-group-add $\Rightarrow{ }^{\prime} v($ infixr $\cdot 70)$

and $V \quad::{ }^{\prime} v$ set

and $T \quad:{ }^{\prime} v \Rightarrow{ }^{\prime} v$

+ assumes endomorph: $\operatorname{Im} G \subseteq V$

locale FGModuleIso $=$ FGModuleHom G smult $V$ smult ${ }^{\prime} T$

for $G \quad::{ }^{\prime} g:$ :group-add set

and smult :: ('f::field, ' g) aezfun $\Rightarrow{ }^{\prime} v:: a b$-group-add $\Rightarrow{ }^{\prime} v(\mathbf{i n f i x r} \cdot 70)$

and $V \quad:$ ' $^{\prime} v$ set

and smult':: ('f, 'g) aezfun $\Rightarrow$ 'w::ab-group-add $\Rightarrow{ }^{\prime} w(\mathbf{i n f i x r} \star 70)$

and $T \quad:{ }^{\prime} v \Rightarrow{ }^{\prime} w$

+ fixes $W \quad:{ }^{\prime} w$ set

assumes bijective: bij-betw $T V W$

abbreviation (in FGModule) isomorphic ::

$\left(\left(' f,{ }^{\prime} g\right)\right.$ aezfun $\Rightarrow$ 'w::ab-group-add $\left.\Rightarrow{ }^{\prime} w\right) \Rightarrow{ }^{\prime} w$ set $\Rightarrow$ bool

where isomorphic smult' $W \equiv(\exists$ T. FGModuleIso G smult $V$ smult' $T W)$

\subsubsection{Basic facts}

context FGModule

begin

lemma trivial-FGModuleHom :

assumes $\bigwedge r . r \in F G \Longrightarrow$ smult' $^{\prime} \mathrm{r}=0$

shows FGModuleHom $G$ smult $V$ smult' 0

$\langle$ proof $\rangle$ 
lemma FGModHom-idhom : FGModuleHom G smult $V$ smult $(i d \downarrow V)$

$\langle$ proof $\rangle$

lemma VecHom-GMap-is-FGModuleHom :

fixes smult' :: ('f, 'g) aezfun $\Rightarrow$ 'w::ab-group-add $\Rightarrow{ }^{\prime} w($ infixr $\star 70)$

and $\quad f_{s m u l t}::{ }^{\prime} f \Rightarrow{ }^{\prime} w \Rightarrow{ }^{\prime} w($ infixr $\sharp \star 70)$

and Gmult' ::' $g \Rightarrow{ }^{\prime} w \Rightarrow{ }^{\prime} w($ infixr $* \star 70)$

defines fsmult $^{\prime}$ : fsmult ${ }^{\prime} \equiv$ aezfun-scalar-mult.fsmult smult ${ }^{\prime}$

and Gmult': Gmult Gezfun-scalar-mult.Gmult smult $^{\prime}$

assumes hom : VectorSpaceHom fsmult $V$ fsmult' $T$

and Im-W: FGModule $G$ smult' $W T$ ' $V \subseteq W$

and $\quad G$-map : ^gv. $g \in G \Longrightarrow v \in V \Longrightarrow T(g * \cdot v)=g * \star(T v)$

shows FGModuleHom $G$ smult $V$ smult' $T$

$\langle$ proof $\rangle$

lemma VecHom-GMap-on-fbasis-is-FGModuleHom :

fixes smult' $\quad::\left({ }^{\prime} f,{ }^{\prime} g\right)$ aezfun $\Rightarrow$ 'w::ab-group-add $\Rightarrow{ }^{\prime} w(\mathbf{i n f i x r} \star 70)$

and fsmult' :: 'f $\Rightarrow{ }^{\prime} w \Rightarrow{ }^{\prime} w$ (infixr $\left.\sharp \star 70\right)$

and Gmult' $\quad:{ }^{\prime} g \Rightarrow{ }^{\prime} w \Rightarrow{ }^{\prime} w($ infixr $* \star 70)$

and flincomb':: 'f list $\Rightarrow{ }^{\prime} w$ list $\Rightarrow{ }^{\prime} w$ (infixr $\left.\bullet \sharp \star 70\right)$

defines fsmult' $:$ fsmult $^{\prime} \equiv$ aezfun-scalar-mult.fsmult smult ${ }^{\prime}$

and Gmult G $^{\prime} \quad$ Gmult ${ }^{\prime} \equiv$ aezfun-scalar-mult.Gmult smult ${ }^{\prime}$

and flincomb $b^{\prime}$ : flincomb ${ }^{\prime} \equiv$ aezfun-scalar-mult.flincomb smult ${ }^{\prime}$

assumes fbasis : fbasis-for $V$ vs

and hom : VectorSpaceHom fsmult $V$ fsmult ${ }^{\prime} T$

and $\quad I m-W \quad:$ FGModule $G$ smult' $W T$ ' $V \subseteq W$



shows FGModuleHom $G$ smult $V$ smult' $T$

$\langle$ proof $\rangle$

end

context FGModuleHom

begin

abbreviation fsmult' :: 'f $\Rightarrow{ }^{\prime} w \Rightarrow^{\prime} w$ (infixr $\left.\sharp \star ~ 70\right)$

where fsmult $^{\prime} \equiv$ aezfun-scalar-mult.fsmult smult ${ }^{\prime}$

abbreviation Gmult $^{\prime}::{ }^{\prime} g \Rightarrow{ }^{\prime} w \Rightarrow{ }^{\prime} w$ (infixr $\left.* \star 70\right)$

where Gmult $^{\prime} \equiv$ aezfun-scalar-mult.Gmult smult ${ }^{\prime}$

lemmas supp $\quad=$ supp

lemmas additive $\quad=$ additive

lemmas $F G$-map $\quad=R$-map

lemmas $F G$-fddo-closed $\quad=F G$-fdd0-closed

lemmas fsmult-smult-domain-comm $=f_{\text {smult-smult-comm }}$

lemmas GSubspace-Ker $=$ RSubmodule-Ker

lemmas Ker-Im-iff = Ker-Im-iff

lemmas Ker0-imp-inj-on =Ker0-imp-inj-on 


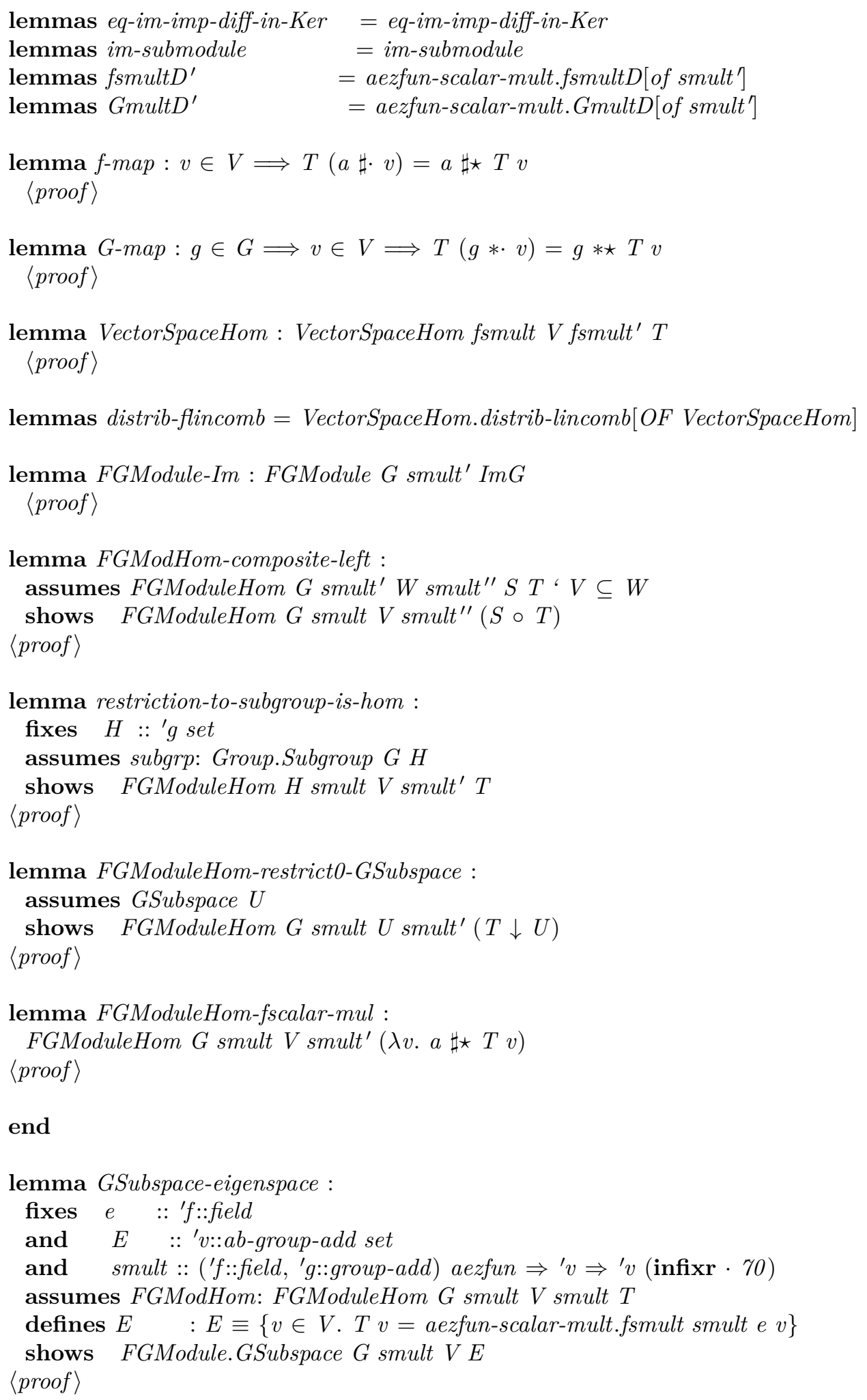




\subsubsection{Basic facts about endomorphisms}

lemma RModuleEnd-over-group-ring-is-FGModuleEnd :

fixes $G \quad::{ }^{\prime} g::$ group-add set

and smult :: ('f::field, 'g) aezfun $\Rightarrow{ }^{\prime} v:: a b$-group-add $\Rightarrow{ }^{\prime} v$

assumes $G$ : Group $G$ and endo: RModuleEnd (Group.group-ring G) smult $V T$

shows FGModuleEnd $G$ smult $V T$

$\langle$ proof $\rangle$

lemma (in FGModule) VecEnd-GMap-is-FGModuleEnd :

assumes endo: VectorSpaceEnd fsmult $V T$

and $\quad G$-map: $\bigwedge g$ v. $g \in G \Longrightarrow v \in V \Longrightarrow T(g * \cdot v)=g * \cdot(T v)$

shows FGModule End $G$ smult $V T$

$\langle$ proof $\rangle$

lemma (in FGModule) GEnd-inner-dirsum-el-decomp-nth :

$\llbracket \forall U \in$ set $U$ s. GSubspace $U$; add-independentS Us; $n<$ length Us 》

$\langle$ proof $\rangle$

$\Longrightarrow$ FGModuleEnd G smult $(\bigoplus U \leftarrow U s . U)(\bigoplus U s \downarrow n)$

context FGModuleEnd

begin

lemma RModuleEnd : RModuleEnd ActingGroup.group-ring smult $V T$ $\langle$ proof $\rangle$

lemma VectorSpaceEnd : VectorSpaceEnd fsmult $V T$ $\langle$ proof $\rangle$

lemmas proj-decomp = RModuleEnd.proj-decomp[OF RModuleEnd $]$

lemmas GSubspace-Ker = GSubspace-Ker

lemmas FGModuleHom-restrict0-GSubspace = FGModuleHom-restrict0-GSubspace

end

\subsubsection{Basic facts about isomorphisms}

context FGModuleIso

begin

lemmas VectorSpaceHom = VectorSpaceHom

abbreviation invT $\equiv($ the-inv-into $V T) \downarrow W$

lemma RModuleIso : RModuleIso FG smult $V$ smult' $T W$

$\langle$ proof $\rangle$

lemmas $\operatorname{Im} G=$ RModuleIso.ImG $[$ OF RModuleIso $]$

lemma FGModuleIso-restrict0-GSubspace : 


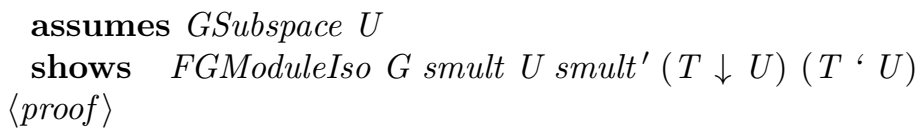

lemma isomorphic-sym : FGModule.isomorphic G smult' $W$ smult $V$ $\langle$ proof $\rangle$

lemma isomorphic-trans :

FGModule.isomorphic $G$ smult' $W$ smult $^{\prime \prime} X$ $\langle$ proof $\rangle$

$\Longrightarrow$ FGModule.isomorphic G smult $V$ smult" $X$

lemma isomorphic-to-zero-left : $V=0 \Longrightarrow W=0$ $\langle$ proof $\rangle$

lemma isomorphic-to-zero-right : $W=0 \Longrightarrow V=0$ $\langle$ proof $\rangle$

lemma isomorphic-to-irr-right' :

assumes $\bigwedge U$. FGModule.GSubspace G smult' $W U \Longrightarrow U=0 \vee U=W$ shows $\bigwedge U$. GSubspace $U \Longrightarrow U=0 \vee U=V$

$\langle$ proof $\rangle$

end

context FGModule

begin

lemma isomorphic-sym :

isomorphic smult' $W \Longrightarrow$ FGModule.isomorphic $G$ smult' $W$ smult $V$ $\langle$ proof $\rangle$

lemma isomorphic-trans :

isomorphic smult' $W \Longrightarrow$ FGModule.isomorphic $G$ smult' $W$ smult ${ }^{\prime \prime} X$ $\langle$ proof $\rangle$ $\Longrightarrow$ isomorphic smult" $X$

lemma isomorphic-to-zero-left : $V=0 \Longrightarrow$ isomorphic smult ${ }^{\prime} W \Longrightarrow W=0$ $\langle$ proof $\rangle$

lemma isomorphic-to-zero-right : isomorphic smult' $0 \Longrightarrow V=0$ 
$\langle$ proof $\rangle$

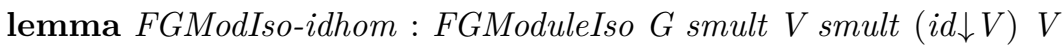

$\langle$ proof $\rangle$

lemma isomorphic-refl : isomorphic smult $V\langle$ proof $\rangle$

end

\subsubsection{Hom-sets}

definition FGModuleHomSet ::

'g::group-add set $\Rightarrow\left(\left({ }^{\prime} f::\right.\right.$ field,,$\left.^{\prime} g\right)$ aezfun $\Rightarrow$ 'v::ab-group-add $\left.\Rightarrow{ }^{\prime} v\right) \Rightarrow{ }^{\prime} v$ set

$\Rightarrow\left((' f, ' g)\right.$ aezfun $\Rightarrow{ }^{\prime} w:: a b$-group-add $\left.\Rightarrow{ }^{\prime} w\right) \Rightarrow{ }^{\prime} w$ set

$\Rightarrow\left({ }^{\prime} v \Rightarrow{ }^{\prime} w\right)$ set

where FGModuleHomSet $G$ fgsmult $V$ fgsmult' $W$

$\equiv\{T$. FGModuleHom $G$ fgsmult $V$ fgsmult' $T\} \cap\{T . T$ ' $V \subseteq W\}$

lemma FGModuleHomSetI :

FGModuleHom $G$ fgsmult $V$ fgsmult' $T \Longrightarrow T$ ' $V \subseteq W$

$\langle$ proof $\rangle$

$\Longrightarrow T \in$ FGModuleHomSet $G$ fgsmult $V$ fgsmult' $W$

lemma FGModuleHomSetD-FGModuleHom :

$T \in$ FGModuleHomSet $G$ fgsmult $V$ fgsmult' $W$

$\langle$ proof $\rangle$

$\Longrightarrow$ FGModuleHom $G$ fgsmult $V$ fgsmult ${ }^{\prime} T$

lemma FGModuleHomSetD-Im :

$T \in$ FGModuleHomSet $G$ fgsmult $V$ fgsmult' $W \Longrightarrow T$ ' $V \subseteq W$

$\langle$ proof $\rangle$

context FGModule

begin

lemma FGModuleHomSet-is-Gmaps-in-VectorSpaceHomSet :

fixes smult' :: ('f, 'g) aezfun $\Rightarrow$ 'w::ab-group-add $\Rightarrow$ 'w (infixr $\star 70)$

and $\quad f_{s m u l t}::{ }^{\prime} f \Rightarrow{ }^{\prime} w \Rightarrow{ }^{\prime} w($ infixr $\sharp \star 70)$

and Gmult $:^{\prime}{ }^{\prime} g \Rightarrow{ }^{\prime} w \Rightarrow{ }^{\prime} w($ infixr $* \star 70)$

defines fsmult' $:$ fsmult ${ }^{\prime} \equiv$ aezfun-scalar-mult.fsmult smult ${ }^{\prime}$

and $\quad$ Gmult ${ }^{\prime}$ : Gmult' $\equiv$ aezfun-scalar-mult.Gmult smult ${ }^{\prime}$

assumes FGModW : FGModule G smult' $W$

shows FGModuleHomSet $G$ smult $V$ smult ${ }^{\prime} W$

$=\left(\right.$ VectorSpaceHomSet fsmult $V$ fsmult $\left.{ }^{\prime} W\right)$

$\cap\{T . \forall g \in G . \forall v \in V . T(g * \cdot v)=g * \star(T v)\}$

$\langle$ proof $\rangle$

lemma Group-FGModuleHomSet :

fixes smult' $::\left({ }^{\prime} f,{ }^{\prime} g\right)$ aezfun $\Rightarrow$ 'w::ab-group-add $\Rightarrow{ }^{\prime} w($ infixr $\star 70)$ 


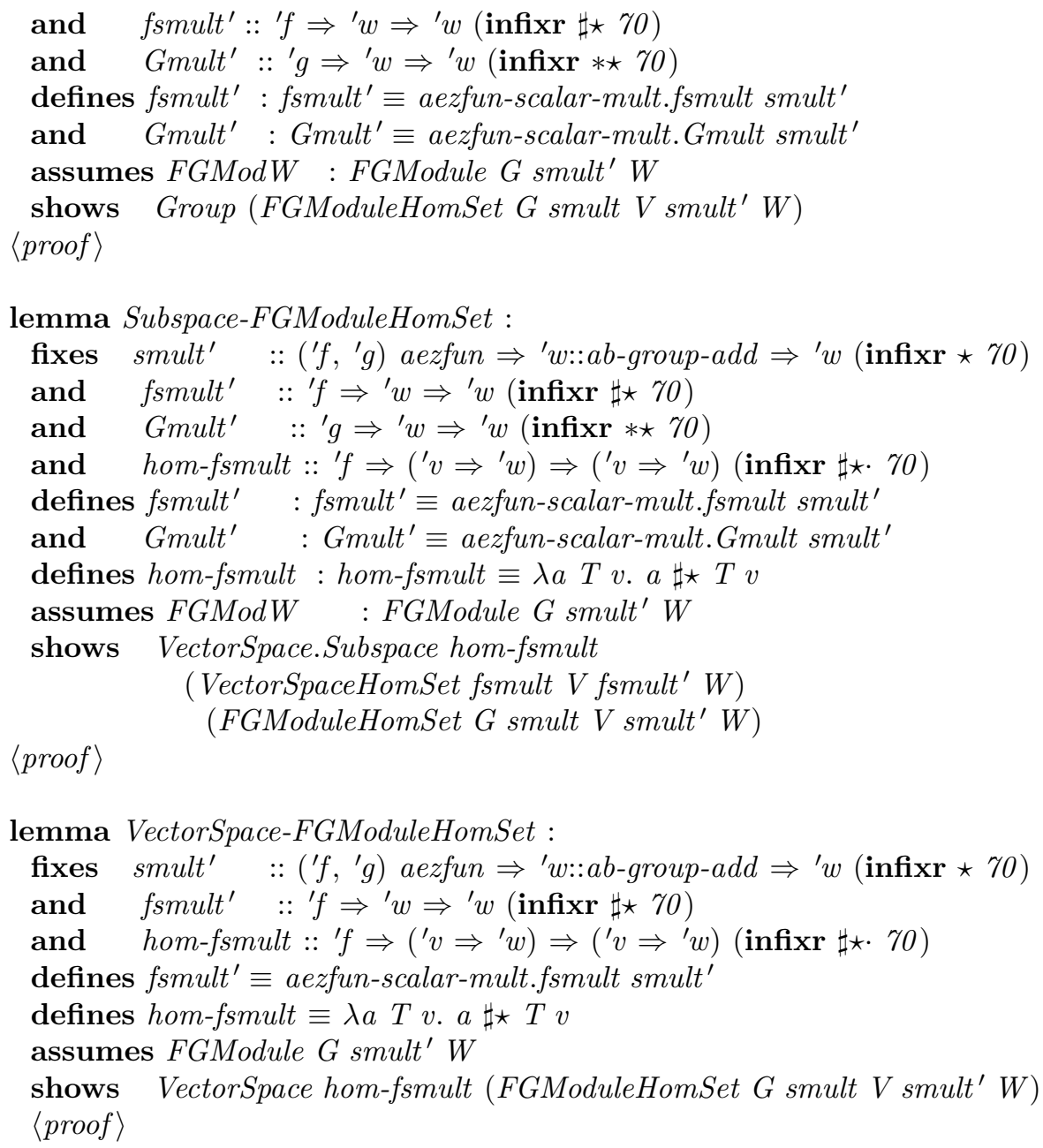

lemma VectorSpace-FGModuleHomSet :

fixes smult' $::\left({ }^{\prime} f,{ }^{\prime} g\right)$ aezfun $\Rightarrow{ }^{\prime} w:: a b$-group-add $\Rightarrow{ }^{\prime} w(\mathbf{i n f i x r} \star 70)$ and fsmult' :: $f \Rightarrow{ }^{\prime} w \Rightarrow^{\prime} w$ (infixr $\left.\sharp \star 70\right)$

end

\subsection{Induced modules}

\subsubsection{Additive function spaces}

definition addfunset ::

'a::monoid-add set $\Rightarrow{ }^{\prime} m::$ monoid-add set $\Rightarrow\left({ }^{\prime} a \Rightarrow{ }^{\prime} m\right)$ set

where addfunset $A M \equiv\{f$. supp $f \subseteq A \wedge$ range $f \subseteq M$

$\wedge(\forall x \in A . \forall y \in A . f(x+y)=f x+f y)\}$

lemma addfunsetI :

$\llbracket$ supp $f \subseteq A$; range $f \subseteq M ; \forall x \in A . \forall y \in A . f(x+y)=f x+f y \rrbracket$ $\langle$ proof $\rangle$

$\Longrightarrow f \in$ addfunset $A M$

lemma addfunsetD-supp $: f \in$ addfunset $A M \Longrightarrow$ supp $f \subseteq A$ $\langle$ proof $\rangle$ 
lemma addfunsetD-range $: f \in$ addfunset $A M \Longrightarrow$ range $f \subseteq M$

$\langle$ proof $\rangle$

lemma addfunsetD-range' $f \in$ addfunset $A M \Longrightarrow f x \in M$ $\langle$ proof $\rangle$

lemma addfunsetD-add :

$\llbracket f \in$ addfunset $A M ; x \in A ; y \in A \rrbracket \Longrightarrow f(x+y)=f x+f y$ $\langle$ proof $\rangle$

lemma addfunset 0 : addfunset $A\left(0::^{\prime} m::\right.$ monoid-add set $)=0$

$\langle$ proof $\rangle$

lemma Group-addfunset :

fixes $M::^{\prime} g:: a b$-group-add set

assumes Group $M$

shows Group (addfunset $R M$ )

$\langle$ proof $\rangle$

5.5.2 Spaces of functions which transform under scalar multiplication by almost-everywhere-zero functions

context aezfun-scalar-mult

begin

definition smultfunset $::{ }^{\prime} g$ set $\Rightarrow\left({ }^{\prime} r,{ }^{\prime} g\right)$ aezfun set $\Rightarrow\left(\left({ }^{\prime} r,{ }^{\prime} g\right)\right.$ aezfun $\left.\Rightarrow{ }^{\prime} v\right)$ set where smultfunset $G F H \equiv\left\{f .\left(\forall a::^{\prime} r . \forall g \in G . \forall x \in F H\right.\right.$.

$f(a \delta \delta g * x)=(a \delta \delta g) \cdot(f x))\}$

lemma smultfunset $D$ :

$\llbracket f \in$ smultfunset $G F H ; g \in G ; x \in F H \rrbracket \Longrightarrow f(a \delta \delta g * x)=(a \delta \delta g) \cdot(f x)$

$\langle$ proof $\rangle$

lemma smultfunsetI :

$\forall a:{ }^{\prime} r . \forall g \in G . \forall x \in F H . f(a \delta \delta g * x)=(a \delta \delta g) \cdot(f x)$

$\Longrightarrow f \in$ smultfunset $G F H$

$\langle$ proof $\rangle$

end

\subsubsection{General induced spaces of functions on a group ring}

context aezfun-scalar-mult

begin

definition indspace ::

'g set $\Rightarrow\left({ }^{\prime} r,^{\prime} g\right)$ aezfun set $\Rightarrow{ }^{\prime} v$ set $\Rightarrow\left(\left({ }^{\prime} r,^{\prime} g\right)\right.$ aezfun $\left.\Rightarrow{ }^{\prime} v\right)$ set

where indspace $G F H V=$ addfunset $F H V \cap$ smultfunset $G F H$ 


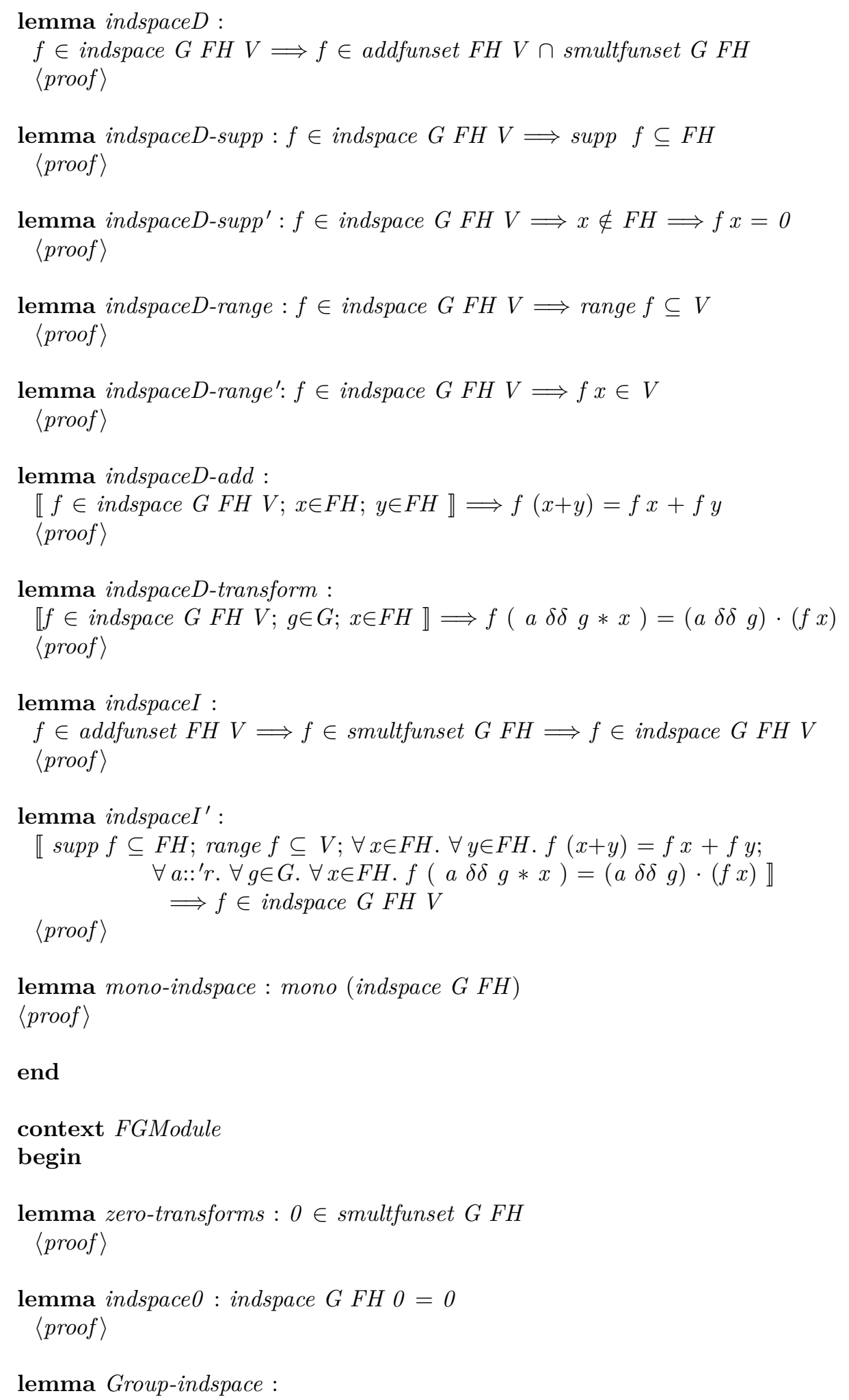






end

\subsubsection{The right regular action}

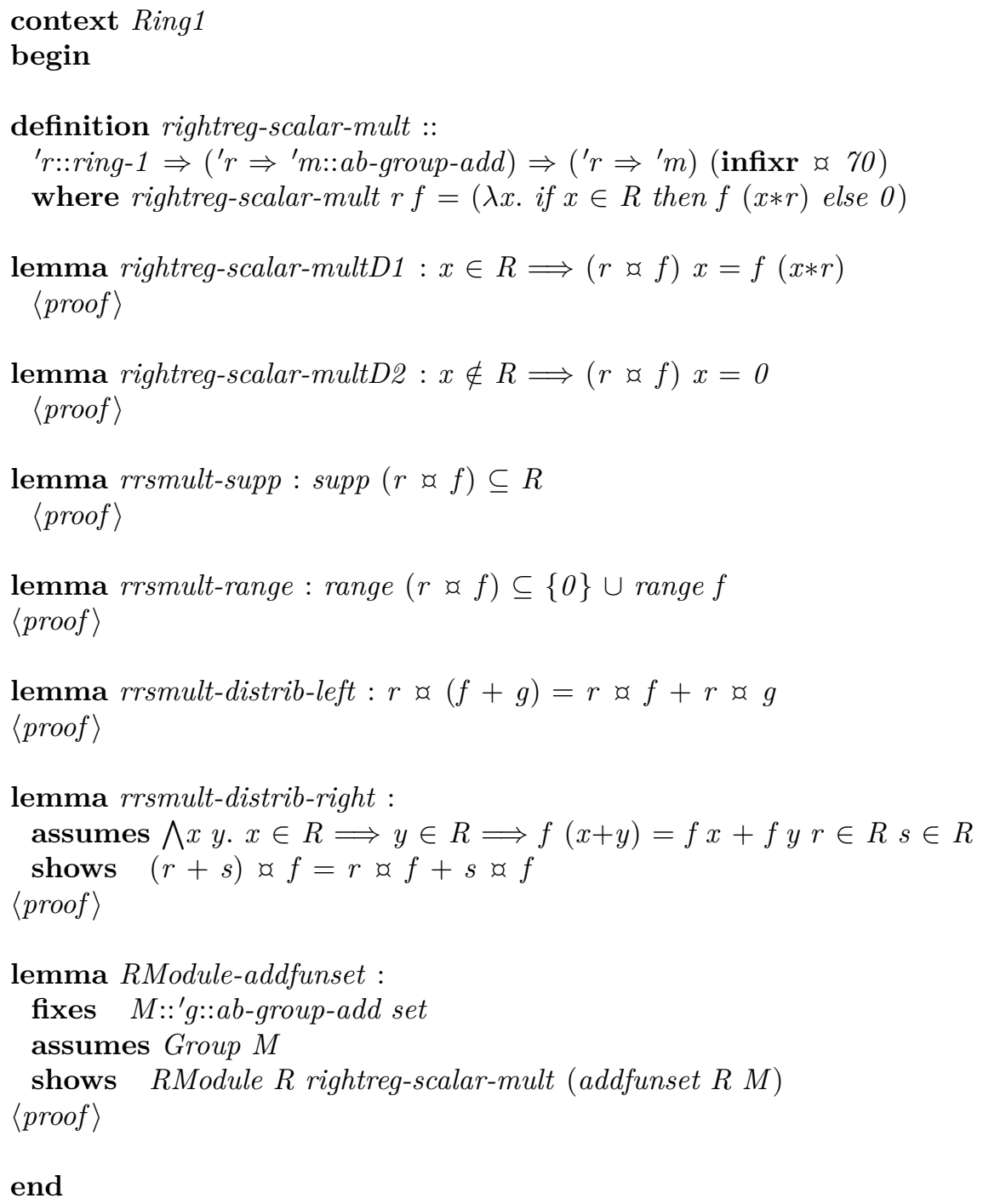

end

\subsubsection{Locale and basic facts}

In the following locale, $G$ is a subgroup of $H, V$ is a module over the group ring for $G$, and the induced space ind $V$ will be shown to be a module over the group ring for $H$ under the right regular scalar multiplication rrsmult. locale InducedFHModule = Supgroup?: Group $H$ 


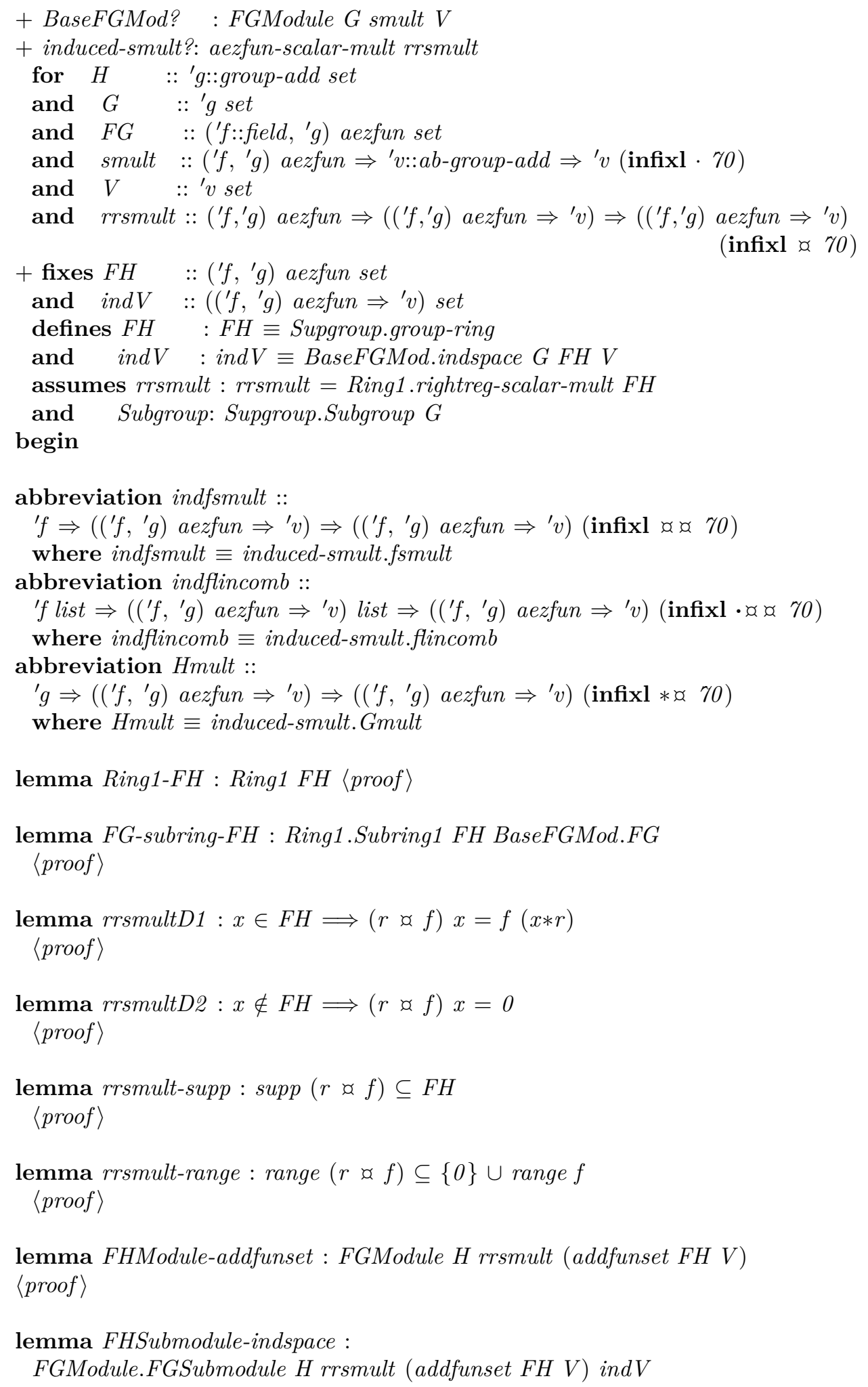


$\langle$ proof $\rangle$

lemma FHModule-indspace : FGModule $H$ rrsmult ind $V$

$\langle$ proof $\rangle$

lemmas fVectorSpace-indspace = FGModule.fVectorSpace [OF FHModule-indspace] lemmas restriction-is-FGModule

$=$ FGModule.restriction-to-subgroup-is-module[OF FHModule-indspace $]$

definition induced-vector :: 'v $\Rightarrow\left(\left(' f,{ }^{\prime} g\right)\right.$ aezfun $\left.\Rightarrow{ }^{\prime} v\right)$

where induced-vector $v \equiv($ if $v \in V$

then $(\lambda y$. if $y \in F H$ then $(F G$-proj $y) \cdot v$ else 0$)$ else 0$)$

lemma induced-vector-apply1 :

$v \in V \Longrightarrow x \in F H \Longrightarrow$ induced-vector $v x=(F G$-proj $x) \cdot v$

$\langle$ proof $\rangle$

lemma induced-vector-apply2 : $v \in V \Longrightarrow x \notin F H \Longrightarrow$ induced-vector $v x=0$ $\langle$ proof $\rangle$

lemma induced-vector-ind $V$ :

assumes $v: v \in V$

shows induced-vector $v \in$ ind $V$

$\langle$ proof $\rangle$

lemma induced-vector-additive :

$v \in V \Longrightarrow v^{\prime} \in V$ $\langle$ proof $\rangle$

$\Longrightarrow$ induced-vector $\left(v+v^{\prime}\right)=$ induced-vector $v+$ induced-vector $v^{\prime}$

lemma hom-induced-vector : FGModuleHom G smult V rrsmult induced-vector $\langle$ proof $\rangle$

lemma indspace-sum-list-fddh:

$\llbracket$ fhs $\neq[$; set $(\operatorname{map}$ snd fhs $) \subseteq H ; f \in$ ind $V \rrbracket$

$\langle$ proof $\rangle$

$\Longrightarrow f\left(\sum(a, h) \leftarrow\right.$ fhs. $\left.a \delta \delta h\right)=\left(\sum(a, h) \leftarrow f h s . f(a \delta \delta h)\right)$

lemma induced-fsmult-conv-fsmult-1ddh :

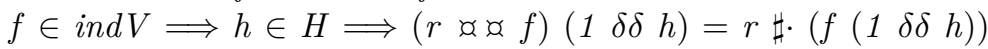

$\langle$ proof $\rangle$

lemma indspace-el-eq-on-1ddh-imp-eq-on-rddh :

assumes HmodG $\subseteq H H=(\bigcup h \in H \bmod G . G+\{h\}) f \in$ ind $V f^{\prime} \in$ ind $V$

$\forall h \in H \bmod G . f(1 \delta \delta h)=f^{\prime}(1 \delta \delta h) h \in H$

shows $f(r \delta \delta h)=f^{\prime}(r \delta \delta h)$

$\langle$ proof $\rangle$

lemma indspace-el-eq : 


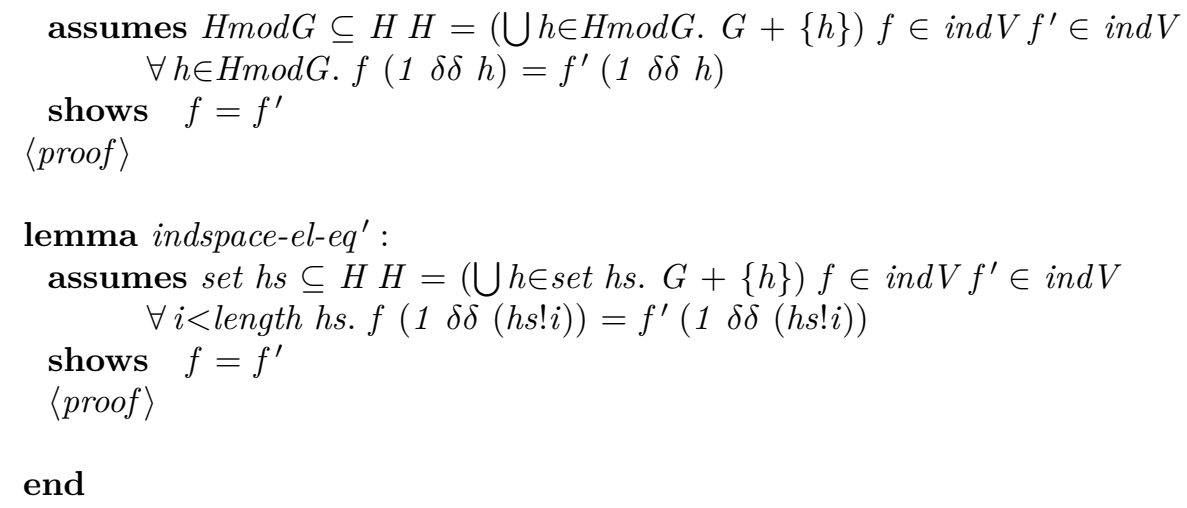

\section{Representations of Finite Groups}

\subsection{Locale and basic facts}

Define a group representation to be a module over the group ring that is finite-dimensional as a vector space. The only restriction on the characteristic of the field is that it does not divide the order of the group. Also, we don't explicitly assume that the group is finite; instead, the good-char assumption implies that the cardinality of $\mathrm{G}$ is not zero, which implies $\mathrm{G}$ is finite. (See lemma good-card-imp-finite.)

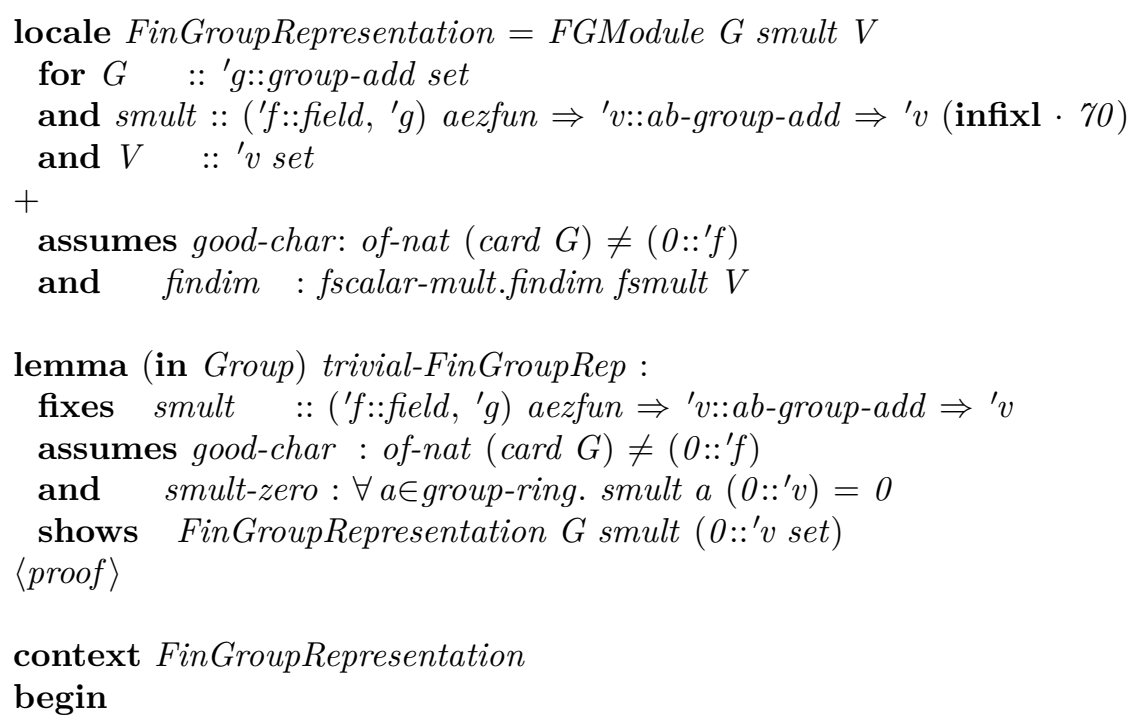


lemmas GSubmodule-GSpan-single = RSubmodule-RSpan-single

lemmas GSpan-single-nonzero = RSpan-single-nonzero

lemma finiteG: finite $G$

$\langle$ proof $\rangle$

lemma FinDim VectorSpace: FinDimVectorSpace fsmult $V$

$\langle$ proof $\rangle$

lemma GSubspace-is-FinGroupRep :

assumes GSubspace $U$

shows FinGroupRepresentation $G$ smult $U$

$\langle$ proof $\rangle$

lemma isomorphic-imp-GRep :

assumes isomorphic smult ${ }^{\prime} W$

shows FinGroupRepresentation $G$ smult ${ }^{\prime} W$

$\langle$ proof $\rangle$

end

\subsection{Irreducible representations}

locale IrrFinGroupRepresentation $=$ FinGroupRepresentation

+ assumes irr: GSubspace $U \Longrightarrow U=0 \vee U=V$

begin

lemmas AbGroup $=$ AbGroup

lemma zero-isomorphic-to-FG-zero :

assumes $V=0$

shows isomorphic $(*)\left(0::\left({ }^{\prime} b,{ }^{\prime} a\right)\right.$ aezfun set $)$

$\langle$ proof $\rangle$

lemma eq-GSpan-single $: v \in V \Longrightarrow v \neq 0 \Longrightarrow V=$ GSpan $[v]$ $\langle$ proof $\rangle$

end

lemma (in Group) trivial-IrrFinGroupRepI :

fixes smult :: ('f::field, 'g) aezfun $\Rightarrow$ ' $v::$ ab-group-add $\Rightarrow{ }^{\prime} v$ assumes of-nat ( card $G) \neq\left(0::^{\prime} f\right)$

and $\quad \forall a \in$ group-ring. smult $a\left(0::^{\prime} v\right)=0$

shows IrrFinGroupRepresentation G smult ( $0:^{\prime}$ 'v set)

$\langle$ proof $\rangle$

lemma (in Group) trivial-IrrFinGroupRepresentation-in-FG :

of-nat $($ card $G) \neq\left(0:: f^{\prime}::\right.$ field $)$

$\Longrightarrow \operatorname{IrrFinGroupRepresentation} G(*)\left(0::\left({ }^{\prime} f,{ }^{\prime} g\right)\right.$ aezfun set $)$ 


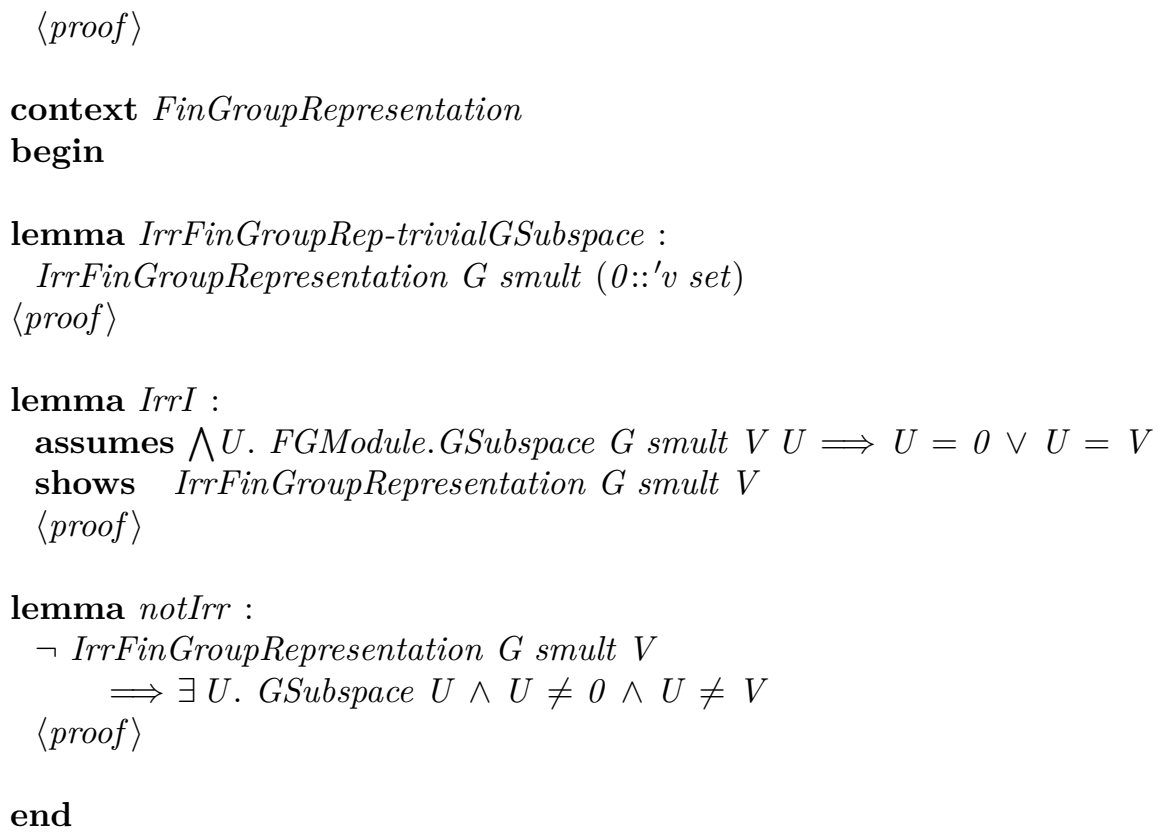

end

\subsection{Maschke's theorem}

\subsubsection{Averaged projection onto a G-subspace}

context FinGroupRepresentation

begin

lemma avg-proj-eq-id-on-right :

assumes VectorSpace fsmult $W$ add-independent $[W, V] v \in V$

defines $P: P \equiv(\bigoplus[W, V] \downarrow 1)$

defines $C P: C P \equiv(\lambda g$. Gmult $(-g) \circ P \circ$ Gmult $g)$

defines $T: T \equiv$ fsmult $(1 /$ ord $G) \circ\left(\sum g \in G\right.$. $\left.C P g\right)$

shows $T v=v$

$\langle$ proof $\rangle$

lemma avg-proj-onto-right :

assumes VectorSpace fsmult $W$ GSubspace $U$ add-independent $S[W, U]$

$$
V=W \oplus U
$$

defines $P: P \equiv(\bigoplus[W, U] \downarrow 1)$

defines $C P: C P \equiv(\lambda g$. Gmult $(-g) \circ P \circ$ Gmult $g)$

defines $T: T \equiv$ fsmult $(1 /$ ord $G) \circ\left(\sum g \in G\right.$. CP $\left.g\right)$

shows $T \cdot V=U$

$\langle$ proof $\rangle$

lemma FGModuleEnd-avg-proj-right :

assumes fSubspace $W$ GSubspace $U$ add-independent $S[W, U] V=W \oplus U$ defines $P: P \equiv(\bigoplus[W, U] \downarrow 1)$

defines $C P: C P \equiv(\lambda g$. Gmult $(-g) \circ P \circ$ Gmult $g)$

defines $T: T \equiv\left(\right.$ fsmult $(1 /$ ord $G) \circ\left(\sum g \in G\right.$. CP $\left.\left.g\right)\right) \downarrow V$ 
shows FGModuleEnd $G$ smult $V T$

$\langle$ proof $\rangle$

lemma avg-proj-is-proj-right :

assumes VectorSpace fsmult $W$ GSubspace $U$ add-independent $S[W, U]$

$V=W \oplus U v \in V$

defines $P: P \equiv(\bigoplus[W, U] \downarrow 1)$

defines $C P: C P \equiv(\lambda g$. Gmult $(-g) \circ P \circ$ Gmult $g)$

defines $T: T \equiv$ fsmult $(1 /$ ord $G) \circ\left(\sum g \in G\right.$. CP $\left.g\right)$

shows $T(T v)=T v$

$\langle$ proof $\rangle$

end

\subsubsection{The theorem}

context FinGroupRepresentation

begin

theorem Maschke :

assumes GSubspace $U$

shows $\exists W$. GSubspace $W \wedge V=W \oplus U$

$\langle$ proof $\rangle$

corollary Maschke-proper :

assumes GSubspace $U U \neq 0 \quad U \neq V$

shows $\exists W$. GSubspace $W \wedge W \neq 0 \wedge W \neq V \wedge V=W \oplus U$

$\langle$ proof $\rangle$

end

\subsubsection{Consequence: complete reducibility}

lemma (in FinGroupRepresentation) notIrr-decompose :

$\neg$ IrrFinGroupRepresentation $G$ smult $V$

$\Longrightarrow \exists U W$. GSubspace $U \wedge U \neq 0 \wedge U \neq V \wedge$ GSubspace $W \wedge W \neq 0$ $\langle$ proof $\rangle$

$\wedge W \neq V \wedge V=U \oplus W$

In the following decomposition lemma, we do not need to explicitly include the condition that all $U$ in set $U s$ are subsets of $V$. (See lemma $A b$ Group-subset-inner-dirsum.)

lemma FinGroupRepresentation-reducible' :

fixes $n:: n a t$

shows $\bigwedge V$. FinGroupRepresentation G fgsmult $V$

$\wedge n=$ FGModule.fdim fgsmult $V$ $\Longrightarrow(\exists U s$. Ball (set Us) (IrrFinGroupRepresentation G fgsmult)

$\langle$ proof $\rangle$

$\wedge(0 \notin$ set $U s) \wedge V=(\bigoplus U \leftarrow U s . U))$ 
theorem (in FinGroupRepresentation) reducible:

$\exists U$ s. $(\forall U \in$ set Us. IrrFinGroupRepresentation G smult $U) \wedge(0 \notin$ set $U s)$ $\langle$ proof $\rangle$

$\wedge V=(\bigoplus U \leftarrow U s . U)$

\subsubsection{Consequence: decomposition relative to a homomomorphism}

lemma (in FinGroupRepresentation) GRepHom-decomp :

fixes $T::^{\prime} v \Rightarrow{ }^{\prime} w:: a b$-group-add

defines $\operatorname{Ker} T: \operatorname{Ker} T \equiv(\operatorname{ker} T \cap V)$

assumes hom : GRepHom smult' $T$ and nonzero: $V \neq 0$

shows $\exists U$. GSubspace $U \wedge V=U \oplus \operatorname{KerT}$

$\langle$ proof $\rangle$

$\wedge$ FGModule.isomorphic G smult $U$ smult' $(T \cdot V)$

\subsection{Schur's lemma}

lemma (in IrrFinGroupRepresentation) Schur-Ker :

GRepHom smult' $T \Longrightarrow T$ ' $V \neq 0 \Longrightarrow$ inj-on $T V$

$\langle$ proof $\rangle$

lemma (in FinGroupRepresentation) Schur-Im :

assumes IrrFinGroupRepresentation G smult' $W$ GRepHom smult' $T$

$T \cdot V \subset W$

$T \cdot V \neq 0$

shows $T^{\prime} V=W$

$\langle$ proof $\rangle$

theorem (in IrrFinGroupRepresentation) Schur1 :

assumes IrrFinGroupRepresentation $G$ smult' $W$

GRepHom smult' $T T^{\prime} V \subseteq W T \cdot V \neq 0$

shows GRepIso smult' $T W$

$\langle$ proof $\rangle$

theorem (in IrrFinGroupRepresentation) Schur2 :

assumes GRep : GRepEnd T

and fdim : fdim $>0$

and alg-closed: $\bigwedge p::^{\prime} b$ poly. degree $p>0 \Longrightarrow \exists$ c. poly $p c=0$

shows $\exists c . \forall v \in V . T v=c \sharp \cdot v$

$\langle$ proof $\rangle$

\subsection{The group ring as a representation space}

\subsubsection{The group ring is a representation space}

lemma (in Group) FGModule-FG :

defines $F G$ : $F G \equiv$ group-ring :: ('f::field, 'g) aezfun set

shows FGModule $G(*) F G$

$\langle$ proof $\rangle$ 


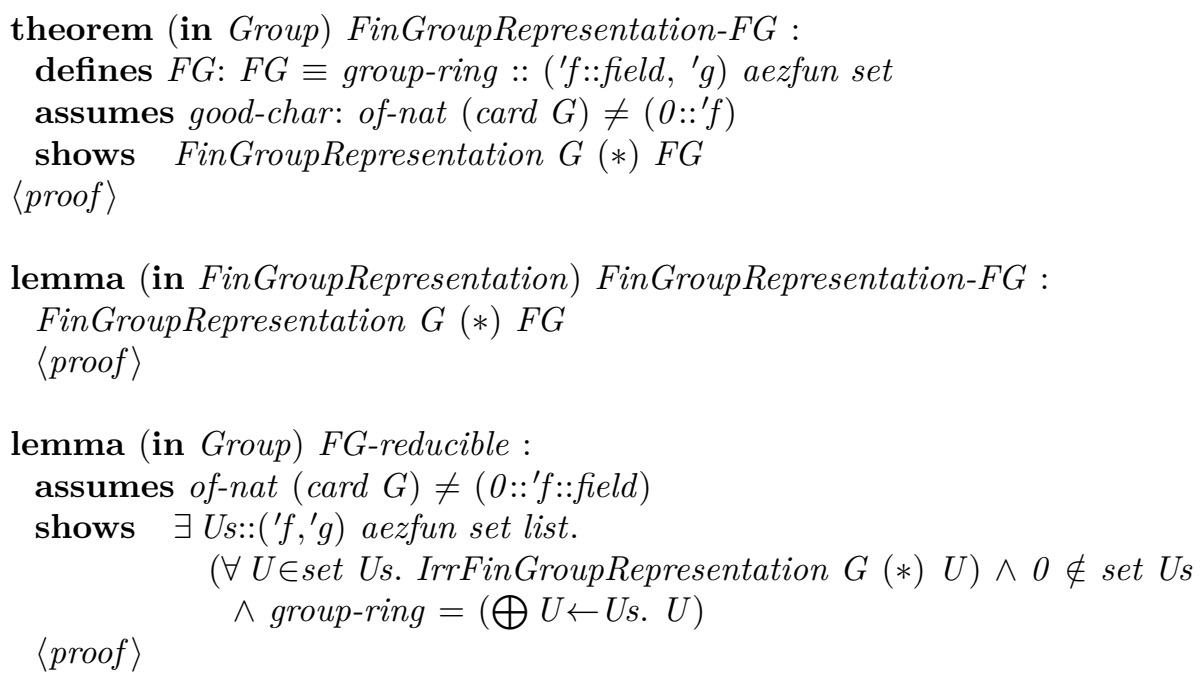

\subsubsection{Irreducible representations are constituents of the group} ring

lemma (in FGModuleIso) isomorphic-to-irr-right : assumes IrrFinGroupRepresentation G smult' $W$ shows IrrFinGroupRepresentation $G$ smult $V$

$\langle$ proof $\rangle$

lemma (in FinGroupRepresentation) IrrGSubspace-iso-constituent :

assumes nonzero : $V \neq 0$

and subsp : $W \subseteq V W \neq 0$ IrrFinGroupRepresentation $G$ smult $W$

and $\quad V$-decomp: $\forall U \in$ set Us. IrrFinGroupRepresentation $G$ smult $U$

$$
0 \notin \text { set } U s \quad V=(\bigoplus U \leftarrow U s . U)
$$

shows $\exists U \in$ set Us. FGModule.isomorphic $G$ smult $W$ smult $U$

$\langle$ proof $\rangle$

theorem (in IrrFinGroupRepresentation) iso-FG-constituent : assumes nonzero : $V \neq 0$

and FG-decomp: $\forall U \in$ set Us. IrrFinGroupRepresentation $G(*) U$

$$
0 \notin \text { set } U s F G=(\bigoplus U \leftarrow U s . U)
$$

shows $\exists U \in$ set Us. isomorphic $(*) U$

$\langle$ proof $\rangle$

\subsection{Isomorphism classes of irreducible representations}

We have already demonstrated that the relation FGModule.isomorphic is reflexive (lemma FGModule.isomorphic-refl), symmetric (lemma FGModule.isomorphic-sym), and transitive (lemma FGModule.isomorphic-trans). In this section, we provide a finite set of representatives for the resulting isomorphism classes of irreducible representations. 




lemma $F G$-constituents-constituents : of-nat $($ card $G) \neq\left(0::^{\prime} f:\right.$ :field $)$ $\langle$ proof $\rangle$$$
\Longrightarrow(\text { group-ring::('f,'g) aezfun set })=(\bigoplus U \leftarrow F G \text {-constituents. } U)
$$

definition GIrrRep-repset $\equiv 0 \cup$ set (remisodups FG-constituents)

lemma finite-GIrrRep-repset : finite GIrrRep-repset $\langle$ proof $\rangle$

lemma all-irr-GIrrRep-repset : assumes of-nat $($ card $G) \neq\left(0::^{\prime} f::\right.$ field $)$ shows $\forall U \in($ GIrrRep-repset::('f,'g) aezfun set set $)$. $\langle$ proof $\rangle$ IrrFinGroupRepresentation $G(*) U$ 
lemma isodistinct-GIrrRep-repset :

defines GIRRS $\equiv$ GIrrRep-repset :: (' $f::$ field, $\left.{ }^{\prime} g\right)$ aezfun set set assumes of-nat (card $G) \neq\left(0::{ }^{\prime} f\right) V \in$ GIRRS $W \in$ GIRRS $V \neq W$ shows $\neg($ FGModule.isomorphic $G(*) V(*) W)$ $\langle$ proof $\rangle$

end

lemma (in FGModule) iso-in-list-imp-iso-in-remisodups : $\exists U \in$ set Us. isomorphic $(*) U$ $\langle$ proof $\rangle$ $\Longrightarrow \exists U \in$ set (ActingGroup.remisodups Us). isomorphic (*) U

lemma (in IrrFinGroupRepresentation) iso-to-GIrrRep-rep : $\exists U \in$ Acting Group.GIrrRep-repset. isomorphic $(*) U$ $\langle$ proof $\rangle$

theorem (in Group) iso-class-reps :

defines GIRRS $\equiv$ GIrrRep-repset :: ('f::field,'g) aezfun set set assumes of-nat ( card $G) \neq(0:: ' f)$

shows finite GIRRS

$\forall U \in$ GIRRS. IrrFinGroupRepresentation $G(*) U$

$\bigwedge U W . \llbracket U \in G I R R S ; W \in G I R R S ; U \neq W \rrbracket$ $\Longrightarrow \neg($ FGModule.isomorphic $G(*) U(*) W$ )

$\bigwedge$ fgsmult $V$. IrrFinGroupRepresentation $G$ fgsmult $V$ $\langle$ proof $\rangle$ $\Longrightarrow \exists U \in G I R R S$. FGModule.isomorphic G fgsmult $V(*) U$

\subsection{Induced representations}

\subsubsection{Locale and basic facts}

locale InducedFinGroupRepresentation = Supgroup?: Group $H$

+ BaseRep?: FinGroupRepresentation G smult $V$

+ induced-smult?: aezfun-scalar-mult rrsmult

for $H \quad::{ }^{\prime} g::$ group-add set

and $G \quad::{ }^{\prime} g$ set

and smult :: ('f::field, 'g) aezfun $\Rightarrow{ }^{\prime} v::$ ab-group-add $\Rightarrow{ }^{\prime} v($ infixl $\cdot 70)$

and $V \quad::$ 'v set

and rrsmult :: ('f,'g) aezfun $\Rightarrow\left(\left(' f,{ }^{\prime} g\right)\right.$ aezfun $\left.\Rightarrow^{\prime} v\right)$

$\Rightarrow\left(\left(' f,,^{\prime} g\right)\right.$ aezfun $\left.\Rightarrow^{\prime} v\right)($ infixl $\ltimes 70)$

+ fixes $F H \quad::\left({ }^{\prime} f,{ }^{\prime} g\right)$ aezfun set

and ind $V \quad::\left(\left({ }^{\prime} f,{ }^{\prime} g\right)\right.$ aezfun $\left.\Rightarrow{ }^{\prime} v\right)$ set

defines $F H \quad: F H \equiv$ Supgroup.group-ring

and $\quad$ ind $V \quad$ indV $\equiv$ BaseRep.indspace $G F H V$

assumes rrsmult $\quad:$ rrsmult $=$ Ring1.rightreg-scalar-mult $F H$

and good-ordSupgrp: of-nat $($ card $H) \neq\left(0::^{\prime} f\right)$

and Subgroup : Supgroup.Subgroup $G$

sublocale InducedFin GroupRepresentation < InducedFHModule 


$$
\langle\text { proof }\rangle
$$

context InducedFinGroupRepresentation begin

abbreviation $\operatorname{ord} H::$ 'f where $\operatorname{ord} H \equiv$ of-nat (card $H$ )

abbreviation is-Vfbasis $\equiv$ fbasis-for $V$

abbreviation GRepHomSet $\equiv$ FGModuleHomSet G smult V

abbreviation HRepHom $\equiv$ FGModuleHom H rrsmult indV

abbreviation HRepHomSet $\equiv$ FGModuleHomSet H rrsmult indV

lemma finiteSupgroup: finite $H$

$\langle$ proof $\rangle$

lemma FinGroupSupgroup : FinGroup $H$ $\langle$ proof $\rangle$

lemmas fVectorSpace $\quad=$ fVectorSpace

lemmas FinDimVectorSpace = FinDimVectorSpace

lemmas ex-rcoset-replist-hd0

$=$ Fin Group.ex-rcoset-replist-hdo[OF FinGroupSupgroup Subgroup $]$

end

\subsubsection{A basis for the induced space}

context InducedFinGroupRepresentation

begin

abbreviation negHorbit-list $\equiv$ induced-smult.negGorbit-list

lemmas ex-rcoset-replist

= FinGroup.ex-rcoset-replist $[$ OF FinGroupSupgroup Subgroup]

lemmas length-negHorbit-list = induced-smult.length-negGorbit-list

lemmas length-negHorbit-list-sublist $=$ induced-smult.length-negGorbit-list-sublist

lemmas negHorbit-list-ind $V \quad=$ FGModule.negGorbit-list-V[OF FHMod-

ule-indspace]

lemma flincomb-Horbit-induced-veclist-reduce :

fixes vs $\quad:{ }^{\prime} v$ list

and $h s \quad:: ' g$ list

defines hfvss : hfvss $\equiv$ negHorbit-list hs induced-vector vs

assumes vs : set vs $\subseteq V$ and $i: i<$ length hs

and scalars : list-all2 ( $\lambda$ rs ms. length $r s=$ length $m s)$ css hfuss

and rcoset-reps : Supgroup.is-rcoset-replist $G$ hs

shows $(($ concat css $) \cdot \propto \propto \alpha($ concat hfvss $))(1 \delta \delta(h s ! i))=($ css $! i) \cdot \sharp \cdot v s$ $\langle$ proof $\rangle$

lemma indspace-fspanning-set : 


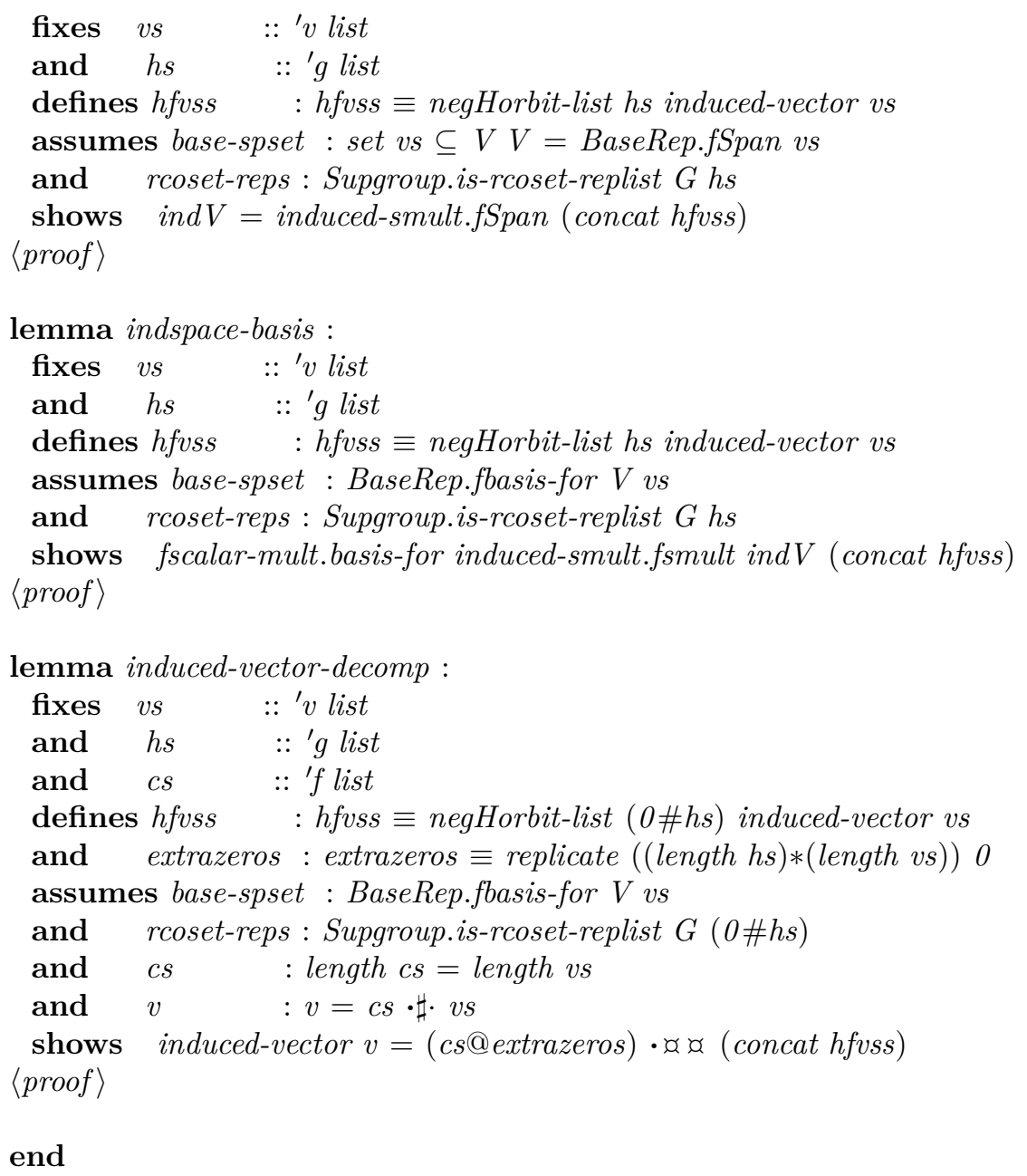

end

\subsubsection{The induced space is a representation space}

context InducedFinGroupRepresentation

begin

lemma indspace-findim :

fscalar-mult.findim induced-smult.fsmult indV $\langle$ proof $\rangle$

theorem FinGroupRepresentation-indspace :

FinGroupRepresentation $H$ rrsmult indV $\langle$ proof $\rangle$

end 


\subsection{Frobenius reciprocity}

\subsubsection{Locale and basic facts}

There are a number of defined objects and lemmas concerning those objects leading up to the theorem of Frobenius reciprocity, so we create a locale to contain it all.

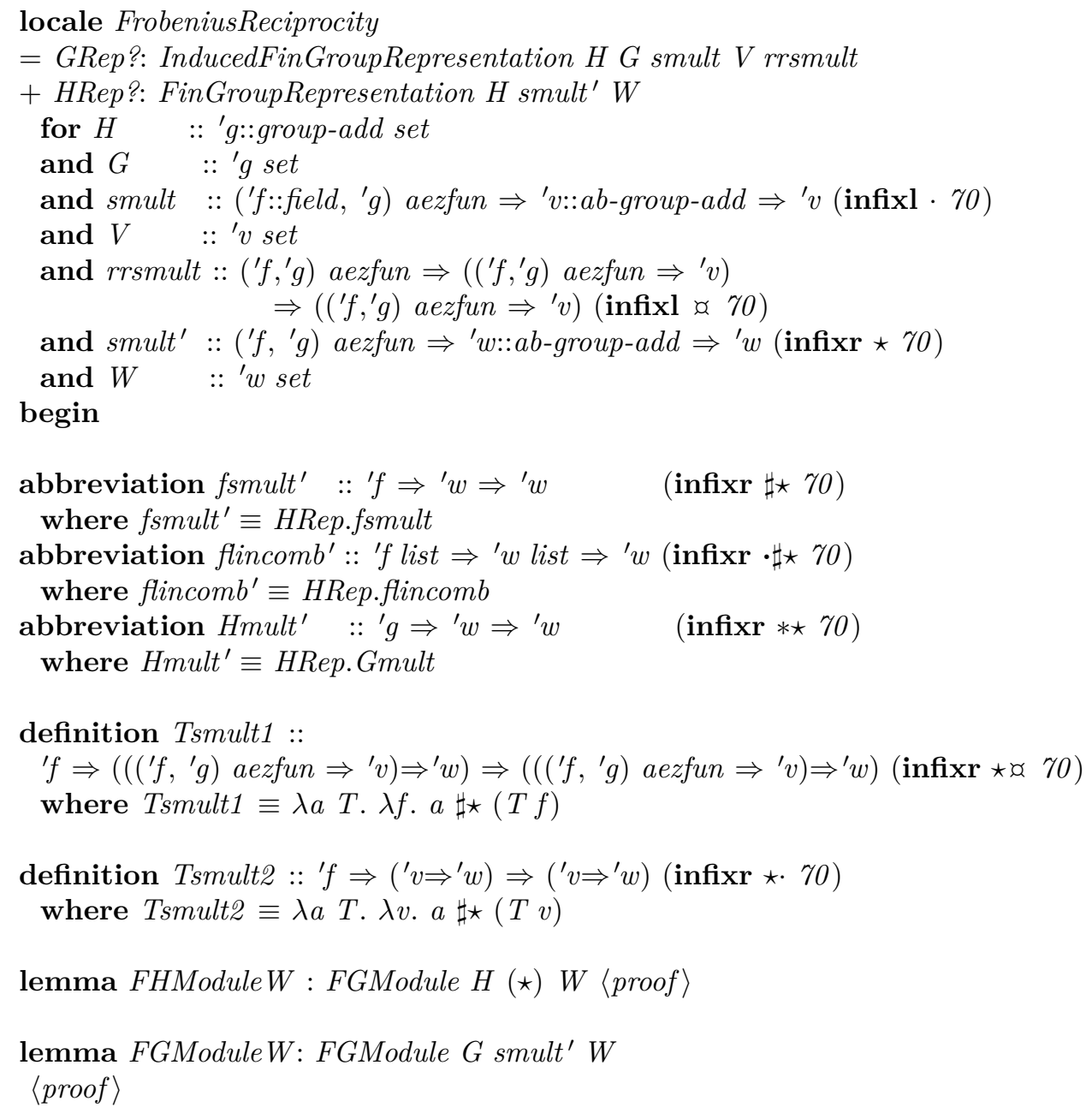

In order to build an inverse for the required isomorphism of Hom-sets, we will need a basis for the induced $H$-space.

definition Vfbasis :: 'v list where Vfbasis $\equiv$ (SOME vs. is-Vfbasis vs)

lemma Vfbasis : is-Vfbasis Vfbasis

$\langle$ proof $\rangle$

lemma Vfbasis- $V$ : set Vfbasis $\subseteq V$

$\langle$ proof $\rangle$ 
definition nonzero-H-rcoset-reps :: 'g list

where nonzero-H-rcoset-reps $\equiv($ SOME hs. Group.is-rcoset-replist $H G(0 \# h s))$

definition H-rcoset-reps :: 'g list where H-rcoset-reps $\equiv 0$ \# nonzero-H-rcoset-reps

lemma H-rcoset-reps : Group.is-rcoset-replist $H G$ H-rcoset-reps

$\langle$ proof $\rangle$

lemma $H$-rcoset-reps- $H$ : set $H$-rcoset-reps $\subseteq H$

$\langle$ proof $\rangle$

lemma nonzero-H-rcoset-reps- $H$ : set nonzero-H-rcoset-reps $\subseteq H$

$\langle$ proof $\rangle$

abbreviation negHorbit-hom Vfbasis :: $\left({ }^{\prime} v \Rightarrow{ }^{\prime} w\right) \Rightarrow{ }^{\prime} w$ list list

where negHorbit-hom Vfbasis $T \equiv$ HRep.negGorbit-list H-rcoset-reps $T$ Vfbasis

lemma negHorbit-Hom-indVfbasis- $W$ :

$T \cdot V \subseteq W \Longrightarrow$ set (concat (negHorbit-hom Vfbasis $T)$ ) $\subseteq$ W

$\langle$ proof $\rangle$

lemma negHorbit-HomSet-indVfbasis- $W$ :

$T \in$ GRepHomSet smult' $W \Longrightarrow$ set (concat (negHorbit-homVfbasis $T)$ ) $\subseteq W$ $\langle$ proof $\rangle$

definition indVfbasis :: (('f, ' $g)$ aezfun $\left.\Rightarrow{ }^{\prime} v\right)$ list list

where indVfbasis $\equiv$ GRep.negHorbit-list H-rcoset-reps induced-vector Vfbasis

lemma indVfbasis :

fscalar-mult.basis-for induced-smult.fsmult indV (concat indVfbasis)

$\langle$ proof $\rangle$

lemma indVfbasis-ind $V:$ hfvs $\in$ set indVfbasis $\Longrightarrow$ set $h f v s \subseteq$ ind $V$ $\langle$ proof $\rangle$

end

\subsubsection{The required isomorphism of Hom-sets}

context FrobeniusReciprocity

begin

The following function will demonstrate the required isomorphism of Homsets (as vector spaces).

definition $\varphi::\left(\left(\left(f,{ }^{\prime} g\right)\right.\right.$ aezfun $\left.\left.\Rightarrow{ }^{\prime} v\right) \Rightarrow{ }^{\prime} w\right) \Rightarrow\left({ }^{\prime} v \Rightarrow{ }^{\prime} w\right)$

where $\varphi \equiv$ restricto $(\lambda T$.T॰ GRep.induced-vector $)($ HRepHomSet smult' $W)$

lemma $\varphi$-im : $\varphi$ 'HRepHomSet $(\star) W \subseteq$ GRepHomSet $(\star) W$

$\langle$ proof $\rangle$ 
end

\subsubsection{The inverse map of Hom-sets}

In this section we build an inverse for the required isomorphism, $\varphi$.

context FrobeniusReciprocity

begin

definition $\psi$-condition $::\left({ }^{\prime} v \Rightarrow{ }^{\prime} w\right) \Rightarrow\left(\left({ }^{\prime} f,{ }^{\prime} g\right)\right.$ aezfun $\left.\left.\Rightarrow{ }^{\prime} v\right) \Rightarrow{ }^{\prime} w\right) \Rightarrow$ bool where $\psi$-condition $T S$

$$
\begin{aligned}
\equiv & \text { VectorSpaceHom induced-smult.fsmult indV fsmult' } S \\
& \wedge \operatorname{map}(\operatorname{map} S) \text { indVfbasis }=\text { negHorbit-hom Vfbasis } T
\end{aligned}
$$

lemma inverse-im-exists' ${ }^{\prime}$

assumes $T \in$ GRepHomSet $(\star) W$

shows $\exists$ ! S. VectorSpaceHom induced-smult.fsmult indV fsmult' $S$

$\wedge$ map $S$ (concat indVfbasis $)=$ concat $($ negHorbit-homVfbasis $T)$

$\langle$ proof $\rangle$

lemma inverse-im-exists :

assumes $T \in$ GRepHomSet $(\star) W$

shows $\exists$ ! S. $\psi$-condition $T S$

$\langle$ proof $\rangle$

definition $\psi::\left({ }^{\prime} v \Rightarrow{ }^{\prime} w\right) \Rightarrow\left(\left(\left(^{\prime} f, ' g\right)\right.\right.$ aezfun $\left.\left.\Rightarrow^{\prime} v\right) \Rightarrow{ }^{\prime} w\right)$

where $\psi \equiv$ restricto $(\lambda T$. THE S. $\psi$-condition T S) (GRepHomSet $(\star) W)$

lemma $\psi D: T \in$ GRepHomSet $(\star) W \Longrightarrow \psi$-condition $T(\psi T)$

$\langle$ proof $\rangle$

lemma $\psi D$-VectorSpaceHom :

$T \in$ GRepHomSet $(\star) W$

$\langle$ proof $\rangle$

lemma $\psi D$-im :

$T \in$ GRepHomSet $(\star) W \Longrightarrow \operatorname{map}(\operatorname{map}(\psi T))$ indVfbasis $\langle$ proof $\rangle$

= aezfun-scalar-mult.negGorbit-list $(\star)$ H-rcoset-reps T Vfbasis

lemma $\psi D$-im-single :

assumes $T \in$ GRepHomSet $(\star) W h \in$ set H-rcoset-reps $v \in$ set Vfbasis shows $\psi T((-h) *$ a $($ induced-vector $v))=(-h) * \star(T v)$

$\langle$ proof $\rangle$

lemma $\psi T-W$ :

assumes $T \in$ GRepHomSet $(\star) W$

shows $\psi T$ 'ind $\subseteq W$ 


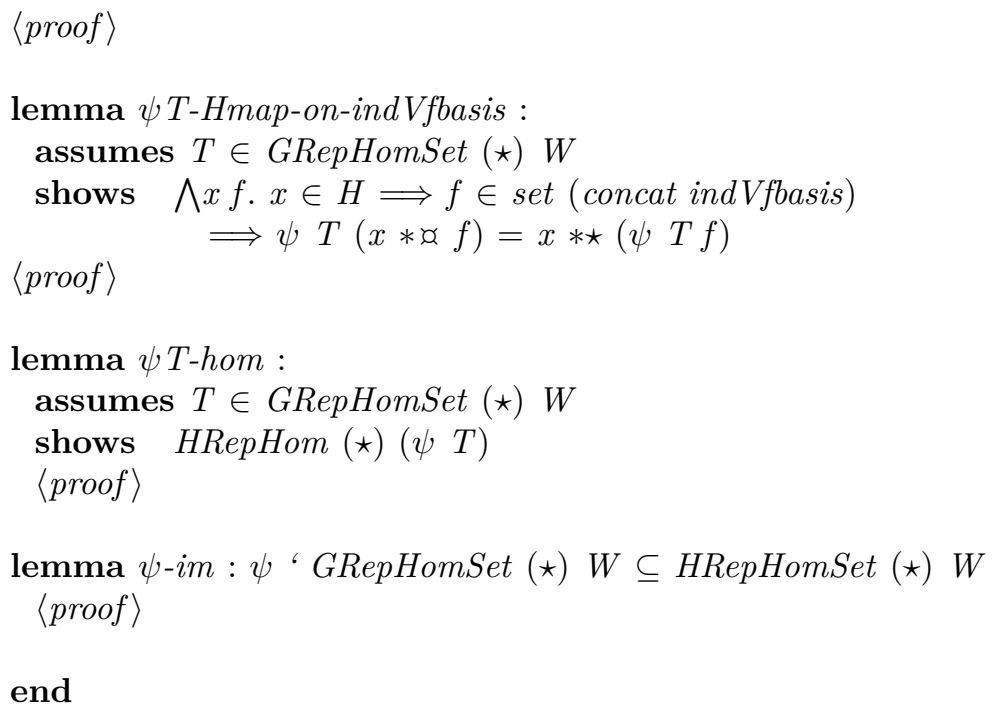

end

\subsubsection{Demonstration of bijectivity}

Now we demonstrate that $\varphi$ is bijective via the inverse $\psi$.

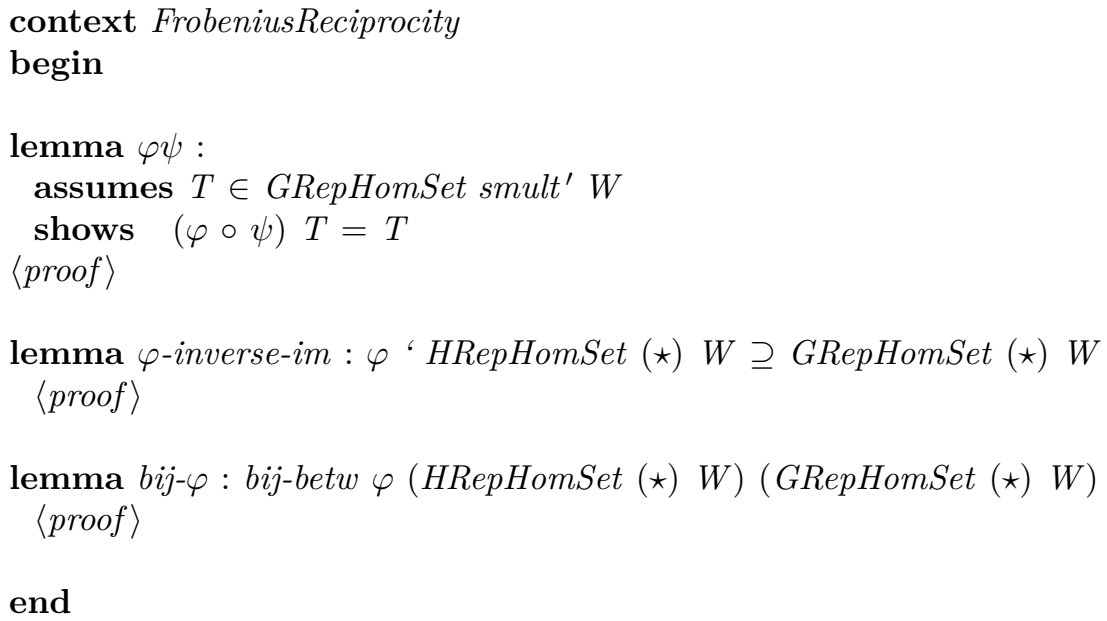

\subsubsection{The theorem}

Finally we demonstrate that $\varphi$ is an isomorphism of vector spaces between the two hom-sets, leading to Frobenius reciprocity.

context FrobeniusReciprocity

begin

lemma VectorSpaceIso- $\varphi$ :

VectorSpaceIso Tsmult1 (HRepHomSet $(\star) W$ ) Tsmult2 $\varphi$ $\langle$ proof $\rangle$ $($ GRepHomSet $(\star) W)$ 
theorem FrobeniusReciprocity :

VectorSpace.isomorphic Tsmult1 (HRepHomSet smult' W) Tsmult2

$\langle$ proof $\rangle$

(GRepHomSet smult' W)

end

end

\section{Bibliography}

[1] S. Axler. Linear Algebra Done Right. Undergraduate Texts in Mathematics. Springer, New York, third edition, 2015.

[2] D. S. Dummit and R. M. Foote. Abstract Algebra. John Wiley \& Sons, New York, second edition, 1999.

[3] G. James and M. Liebeck. Representations and Characters of Groups. Cambridge Mathematical Textbooks. Cambridge University Press, Cambridge, UK, 1993. 